Aston University

Some pages of this thesis may have been removed for copyright restrictions.

If you have discovered material in AURA which is unlawful e.g. breaches copyright, (either yours or that of a third party) or any other law, including but not limited to those relating to patent, trademark, confidentiality, data protection, obscenity, defamation, libel, then please read our Takedown Policy and contact the service immediately 


\title{
SHANNON CAPACITY OF NONLINEAR COMMUNICATION CHANNELS
}

\author{
Mariia Sorokina
}

Doctor of Philosophy

\author{
Aston University
}

March 2014

(C) Mariia Sorokina, 2014

Mariia Sorokina asserts her moral right to be identified as the author of this thesis

This copy of the thesis has been supplied on condition that anyone who consults it is understood to recognise that its copyright rests with its author and that no quotation from the thesis and no information derived from it may be published without appropriate permission or acknowledgement. 


\title{
Aston University
}

\author{
Shannon Capacity of Nonlinear Communication Channels \\ Mariia Sorokina \\ Doctor of Philosophy \\ 2014
}

The exponentially increasing demand on operational data rate has been met with technological advances in telecommunication systems such as advanced multilevel and multidimensional modulation formats, fast signal processing, and research into new different media for signal transmission. Since the current communication channels are essentially nonlinear, estimation of the Shannon capacity for modern nonlinear communication channels is required.

This $\mathrm{PhD}$ research project has targeted the study of the capacity limits of different nonlinear communication channels with a view to enable a significant enhancement in the data rate of the currently deployed fiber networks. In the current study, a theoretical framework for calculating the Shannon capacity of nonlinear regenerative channels has been developed and illustrated on the example of the proposed here regenerative Fourier transform (RFT). Moreover, the maximum gain in Shannon capacity due to regeneration (that is, the Shannon capacity of a system with ideal regenerators - the upper bound on capacity for all regenerative schemes) is calculated analytically. Thus, we derived a regenerative limit to which the capacity of any regenerative system can be compared, as analogue of the seminal linear Shannon limit. A general optimization scheme (regenerative mapping) has been introduced and demonstrated on systems with different regenerative elements: phase sensitive amplifiers and the proposed here multilevel regenerative schemes: the regenerative Fourier transform and the coupled nonlinear loop mirror. 
To my mother 


\section{Acknowledgements}

I am deeply grateful to Professor Sergei Turitsyn, my supervisor, whom I learnt from and who guided and supported me through: for his inspiration, great ideas, wealth of knowledge and patient guidance with intellectual freedom. Dr. Elena Turitsyna, who supported and helped me in times of difficulty. Dr. Stanislav Derevyanko for having given me the opportunity to pursue my $\mathrm{PhD}$ at Aston. Dr. Stylianos Sygletos and Prof. Andrew Ellis for their kind support, immense knowledge and a lot of fruitful discussions. 


\section{Contents}

$\begin{array}{lr}\text { List of Figures } & 8\end{array}$

1 Introduction $\quad 11$

2 Shannon capacity 17

2.1 Introduction . . . . . . . . . . . . . . . . 17

2.2 Before Shannon . . . . . . . . . . . . . . . 18

2.2.1 Nyquist sampling theorem . . . . . . . . . . . . . . . . . . . . . . . . . . .

2.2.2 Hartley's contribution . . . . . . . . . . . . . 19

2.3 Shannon's pioneering work . . . . . . . . . . . 20

2.3 .1 Introduction . . . . . . . . . . . . . 20

2.3.2 Basic components of communication system . . . . . . . 21

2.3.3 Shannon's contributions to information theory . . . . . . 26

2.4 Shannon capacity . . . . . . . . . . . . . . . . 26

2.4.1 Entropy as a measure of information . . . . . . . . . 27

2.4.2 Mutual information ............... . . 29

2.4.2.1 Discrete input - discrete output . . . . . . . . 29

2.4.2.2 Discrete input - continuous output . . . . . . 31

2.4.2.3 Continuous input - continuous output . . . . . 31

2.4.3 Shannon capacity theorem . . . . . . . . . . . . . 32

2.5 Channel capacity and coding . . . . . . . . . . . . . . . . . . . . . . . . . 33

2.6 Communication above the Shannon capacity . . . . . . . . . . . . 34

2.6.1 Numerical calculations of Shannon capacity . . . . . . . . . 35

2.7 Conclusion . . . . . . . . . . . . . . . 36 
3 Shannon capacity of different communication channels 37

3.1 Types of channels . . . . . . . . . . . . . . . 38

3.2 Capacity of linear additive noise channel . . . . . . . . . . 39

3.2.1 Capacity of linear additive white Gaussian noise channel . . . 39

3.2.2 Capacity of band-limited linear AWGN channel . . . . . . 40

3.3 Nonlinear fiber-optic channel . . . . . . . . . . . . . . . 41

3.3.1 Model . . . . . . . . . . . . . . . . . . 41

3.3.2 Simplified channel models . . . . . . . . . . . . 45

3.3.2.1 Constant-intensity modulation with coherent detection ..................... 45

3.3.2.2 Intensity-modulated direct-detection (IMDD) channel ...................... 46 46

3.3.2.3 Partially coherent AWGN . . . . . . . . . . 46

3.3.2.4 Nonlinear phase noise channel . . . . . . . . 47

3.3.2.5 Optical fiber channels with zero average dispersion $\quad 48$

3.3.2.6 Infinite-memory Gaussian noise channel model . . 49

3.4 Conclusion . . . . . . . . . . . . . . . . . 50

4 Shannon capacity of nonlinear regenerative channels 51

4.1 Introduction . . . . . . . . . . . . . . . . . 51

4.2 Regenerative channel . . . . . . . . . . . . . . . . . 52

4.2.1 Numerical verification . . . . . . . . . . . . . . 54

4.3 Regenerative mapping . . . . . . . . . . . . . . . 57

4.3.1 Regenerative functions . . . . . . . . . . . . . . 59

4.3.2 Regenerative Fourier transform model . . . . . . . . . . 63

4.4 Upper bound of regeneration efficiency - capacity of the channel with ideal regenerators . . . . . . . . . . . . . . . . . . . 64

4.4.1 Analytical calculations of the capacity of the regenerative channel . . . . . . . . . . . . . . . . . . . 67

4.4.2 Numerical simulations of the capacity of the regenerative channel 71

4.5 Capacity calculation for regenerative mapping . . . . . . . . . 77

4.5.1 Numerical calculation of the Shannon capacity of RFT channel 78

4.5.1.1 Impact of parameter $b \ldots \ldots$. . . . . . . 80 
4.5.1.2 Optimization of parameter $b \ldots \ldots 83$

4.5.1.3 Impact of parameter $a \ldots \ldots$. . . . . . 84

4.5.1.4 Shannon capacity of the RFT channel . . . . . . . 84

4.5.2 Upper bound of capacity for the RFT mapping . . . . . . . 86

4.6 Conclusion . . . . . . . . . . . . . . . . . . . . 91

5 Design and optimization of phase and amplitude regenerative channels 93

5.1 Introduction . . . . . . . . . . . . . . . . . . . . 93

5.2 Phase regeneration . . . . . . . . . . . . . . 95

5.2.1 Optimization of Cascaded Regenerative Links based on Phase

Sensitive Amplifiers . . . . . . . . . . . . . . . . 96

5.2.2 Simulation Model _. . . . . . . . . . . . . . . 101

5.2.2.1 Optimization of transfer function parameter . . . 103

5.2.3 Optimal packing for cascaded regenerative transmission based on phase sensitive amplifiers . . . . . . . . . . . . 105

5.2.4 Results and discussion . . . . . . . . . . . . . . 108

5.3 Design of multilevel amplitude regenerative system . . . . . . . . 116

5.3 .1 Introduction . . . . . . . . . . . . . . . . 116

5.3 .2 Design ...................... 116

5.3.3 Numerical simulations . . . . . . . . . . . . . . . . . 119

5.4 Multilevel phase and amplitude regeneration . . . . . . . . . . . 123

5.5 Regenerative Fourier Transformation . . . . . . . . . . . . . . . . . . 123

5.5.1 Potential implementation of RFT . . . . . . . . . . . . . 123

5.5.2 Numerical Simulations . . . . . . . . . . . . . . 125

5.6 Conclusions . . . . . . . . . . . . . . . . . 128

6 Conclusion and discussion $\quad 130$

$\begin{array}{lll}7 & \text { List of publications related to the thesis } & 133\end{array}$

$\begin{array}{lr}\text { Bibliography } & 135\end{array}$ 


\section{List of Figures}

1.1 Maximum reported information capacity . . . . . . . . . . . . 9

2.1 Basic components of a communication system . . . . . . . . . . 18

2.2 Basic components of a communication system (expanded version) . . 19

2.3 Basic modulation formats . . . . . . . . . . . . . . . . 19

2.4 The entropy of the binary case . . . . . . . . . . . . 23

2.5 Cutoff rate (for equiprobable $q$-ASK) and capacity . . . . . . . . 30

3.1 Dependence of the Shannon capacity of the linear AWGN channel on channel bandwidth . . . . . . . . . . . . . . . . . 37

4.1 The regenerative channel model . . . . . . . . . . . . . . 49

4.2 Waveforms for noiseless transmission via $R=20$ regenerators $\ldots \quad \ldots 2$

4.3 Waveforms for noisy $(O S N R=10 \mathrm{~dB})$ transmission via $R=20$ regen-

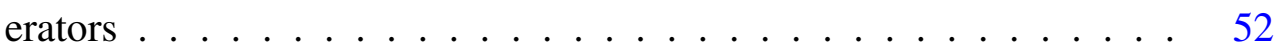

4.4 Waveforms for noisy $(O S N R=10 \mathrm{~dB})$ transmission via $R=5$ regenerators ......................... 53

4.5 Comparison of discrete-time and continuous-time modelling . . . . . 54

4.6 The regenerative sine transfer function $(\mathrm{TF}) \ldots \ldots$. . . . . . . . 56

4.7 Regenerative transfer functions . . . . . . . . . . . . . . 57

4.8 Transmission improvement . . . . . . . . . . . . . . . . . 58

4.9 The regenerative functions - approximation of ideal regenerator . . . 59

4.10 The regenerative channel model . . . . . . . . . . . . . 62

4.11 Optimum cell size . . . . . . . . . . . . . . . . . . . . . . 69

4.12 Optimum input probability . . . . . . . . . . . . . . . . 69

4.13 Regeneration limit. . . . . . . . . . . . . . . . . 70 
4.14 Conditional pdf. . . . . . . . . . . . . . . . . . 75

4.15 Optimum input pmf . . . . . . . . . . . . . . . 77

4.16 Constrained capacity of the two-dimensional RFT channel and the cor-

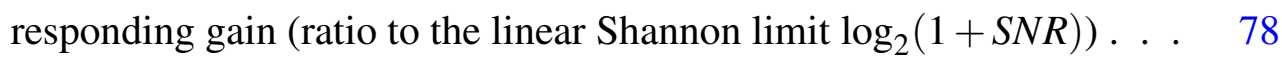

4.17 Optimum value of a) parameter $b$ and b) cell size versus SNR . . . . 79

4.18 Shannon capacity of the RFT channel and the corresponding capacity

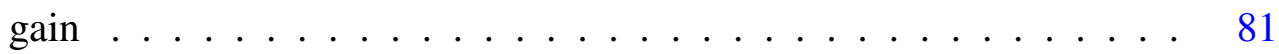

4.19 Optimal input probability . . . . . . . . . . . . . . 82

4.20 Output pdf . . . . . . . . . . . . . . 86

5.1 PSA response . . . . . . . . . . . . . . . . . . . . . . 92

5.2 Periodicity and slope characteristics of the PSA phase transfer function 92

5.3 The PSA transfer function . . . . . . . . . . . . . . 93

5.4 a) PSA phase transfer function for 8-PSK format. The optimal value of the parameter $m_{o p t}=1 / 7$ (shown by green solid line) demonstrates a plateau centered at the alphabet points, whereas a critical choice $m_{c r}=$ 0.33 of the parameter (shown by red dashed line) is expected to lead to poorer regenerative performance. b) Dependence of the optimal and critical values of $m$ and the attraction region's maximum half-width $\Delta_{\text {max }}$ on the order $M$ of the PSK modulation format. . . . . . . . 94

5.5 Schematic diagram . . . . . . . . . . . . . . . . . . 97

5.6 Constellation diagram of 8-PSK . . . . . . . . . . . . . 99

5.7 SER as a function of SNR with cascaded regenerative PSA elements . 101

5.8 The dependence of the SNR improvement due to PSA application . . 102

5.9 The dependence of the SNR improvement due to PSA application . . 102

5.10 Phase symmetric constellations for 8 -symbols . . . . . . . . . . 103

5.11 SERs as a function of SNR for the examined 8-symbol constellation types . . . . . . . . . . . . . . . . . . . . . 104

5.12 Amplitude and phase error for the received signal. . . . . . . . . . 106

5.13 Phase symmetric constellations formats for 16 symbols . . . . . . . 107

5.14 The SERs as a function of SNR for the various types of modulation formats . . . . . . . . . . . . . . . . 108

5.15 Scheme of the coupled NOLM . . . . . . . . . . . . 112 
5.16 Transfer function of the coupled NOLM . . . . . . . . . 113

5.17 Constellation diagrams (normalized to signal power $S=1$ ) for circular

(1) 16-, (2) 64- and (3) 256-QAM . . . . . . . . . . . 115

5.18 Constellation diagrams (normalized to signal power $S=1$ ) for circular

a) 24- and b) 32-QAM . . . . . . . . . . . . . . . . . 116

5.19 Scheme of RFT . . . . . . . . . . . . . . . . . 119

5.20 Constellation diagrams (normalized to signal power $S=1$ ) for rectangular (a) 4-, (b) 16- and (c) 64-QAM . . . . . . . . . . . . . 120

5.21 Constellation diagrams (normalized to signal power $S=1$ ) for rectangular 256-QAM . . . . . . . . . . . . . . . . 121

5.22 SERs as a function of SNR for 16- and 64-QAM ) for rectangular 256-

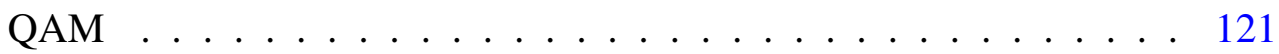




\section{1}

\section{Introduction}

Since Shannon proposed a mathematical theory for communication (1), it became actively used in various scientific disciplines: mathematics, physics, economics, biology, linguistics and other fields of natural and social sciences. His key achievement was a proof that the efficiency of information transmission through a communication channel can be estimated by a single parameter - Shannon capacity, an optimizational functional, where by adapting the input distribution to the channel properties, stochastic and deterministic, one can calculate the maximum error-free transmission rate.

Since Shannon defined the capacity and calculated its value for a linear channel, a few results were obtained for bounds on the nonlinear channel capacity. However, though there are a variety of different nonlinear channel models, only few analytical exact results are obtained. The intensity modulated direct detection channel, the phase noise channel, the fiber-optic channel and the multipath channels are models of high practical importance. Among these conditional probability density function has been derived for only few $(2,3)$, which is necessary for signal optimization. Thus, instead of exact capacity results, a number of lower or upper bounds for capacity were reported (2)-(21).

The increasing demand for data rate drives advances in theoretical studies and engineering technologies. Where information theory enables calculation of the capacity and technology proposes new practical channel types with various transmission characteristics. In the post-Shannon era, following Shannon's ingenious approach to coding, the information theory has been enriched by various coding techniques developed for a variety of applications in an aim to approach the established linear Shannon limit. 
The problem of the discrete representation of a continuous bi-Gaussian distribution resulted in a variety of modulation formats, in particular ring constellations ((22), (23) and references therein) with additional optimization over input probabilities (e. g. iterative polar quantization (24)), which enables high transmission performance (25)(27). Currently, an optimization of 4D modulation formats is widely investigated (see review in (28)).

Key innovations in engineering led to further increases in data rate. Among these, distributed feed-back laser, erbium doped amplifier (EDFA) and Raman amplification, and various modifications of the fiber link as a transmission medium, were further supported by electronic signal processing, optical frequency division multiplexing (OFDM), wavelength division multiplexing (WDM) and time-division multiplexing (OTDM) (see the corresponding capacity increase in Fig. 1.1). In 2009 Charles Kao was awarded a Nobel Prize for the work of 1960 (29), which led to practical implementations of single-mode silica-based optical fiber, which is now responsible for generation of over $90 \%$ of data traffic. Assisted by laser innovations, it has become a foundation of modern communication systems, whereas digital signal processing offers fast and reliable multiplexing techniques for using time, phase, wavelength, and polarization of light, which is reflected in information theory by a number of works devoted to efficient signal coding and packing (e.g. 4D modulation formats (28) and references therein).

As a result, the transmission rate has increased dramatically over past decades, with recent experiments demonstrating capacity of the order of Pbits/s (30). However, as was indicated in a number of studies (30), there has been a continuous succession of ever more demanding services, with current demand dominated by social networking, YouTube, in particular video services, which require 3D ultra-high definition formats, e.g. online streaming of London 2012-Olympics by BBC. Unfortunately, the efficiency of current transmission systems are limited by a number of effects, in particular, noise and nonlinearity. In-fiber nonlinearity is induced from the Kerr effect due to field dependent refractive index change. As a result, the output of a communication channel suffers not only from noise, but also from self-phase modulation (SPM), cross-phase modulation (XPM) and four-wave mixing (FWM) (31)-(34). This significantly reduces the efficiency of transmission channel, though a variety of different mitigation techniques were proposed (35)-(39). Among nonlinear effects, one can distinguish 


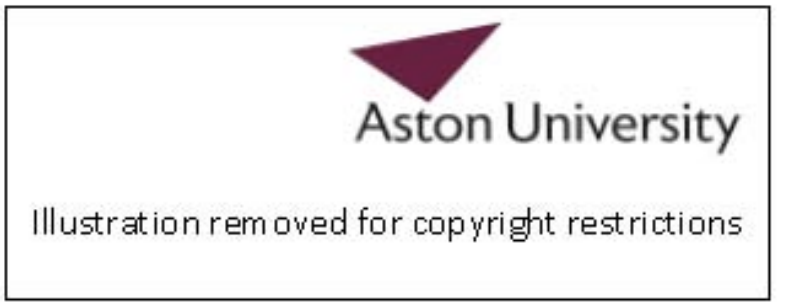

Figure 1.1: Maximum reported information capacity - for single wavelength (diamonds, open symbols for OTDM), WDM (triangles), single and multi-band OFDM (filled circles) and coherent detection (open circles). (taken from (18))

deterministic effects, which, in principle, can be compensated, though the proposed methods (40)-(45) are found to be impractical for compensation of full optical bandwidth. Thus, currently the practical transmission efficiency is limited by nonlinearity and signal processing load, and the corresponding nonlinear limit of fiber-channel capacity was estimated in a number of works, e.g. (18), (30). Moreover, current "hero" experiments show that the capacity approaches the calculated nonlinear limit. The estimations of capacity demands and analysis of current trends show that the estimated capacity crunch is expected in 2020 (30).

Thus, though exact nonlinear Shannon capacity has not been calculated for existing channel models, it was indicated that in case of full nonlinear compensation and signal optimization, the Shannon capacity for static channel models (19) should increase monotonically with signal power $(17,46)$. However, current capacity estimations $((12,18)$ and references therein) predict the limit on capacity growth due to nonlinearity increasing with signal power. In particular, the conventional Gaussian noise signal approximation $(12,18,47)$ shows that for uncompensated unoptimized link the estimated transmission rate vanish with the increase of signal power, whereas in (46) the difference between achievable rate or mutual information (unoptimized Shannon functional), constrained capacity (Shannon functional optimized over the subset of probability distributions), and the Shannon capacity (optimized Shannon functional) 
was stressed. Being in agreement with (46), the lower bound on the Shannon capacity of the nonlinear fiber-optic channel with zero-dispersion was derived (17), showing that the corresponding Shannon capacity increases with growing signal power, both studies highlight the importance of analytical calculation of the optimized Shannon functional - Shannon capacity. Therefore, since there is a variety of different nonlinear channels, the nonlinear Shannon limit remains an open and acute problem.

Hence, there is a surge in search of new techniques for analytical capacity calculations and new methods of nonlinearity mitigation, as well as investigation of additional degrees of freedom for more advanced data multiplexing. The latter resulted in spatialdivision-multiplexing for multicore (48) and multimode (49) fibers. Indeed, it was shown that the new technologies enable a capacity increase a number of times (50), however, such parallel systems are also affected by the nonlinear crosstalk between the cores/modes (50) and require sophisticated DSP.

Also, recent experiments show that employing phase-conjugation one can efficiently mitigate nonlinearity and dispersion (51)-(54), though it reduces the flexibility of the system due to required mid-link optical phase conjugator. This was addressed by proposing different system configurations $(55,56)$. As another example, in (57) the authors proposed to transmit a pair of symmetric phase-conjugated twin waves with additional requirement of dispersion-symmetry along the transmission path, resulting in nonlinearity impairments being cancelled by adding the recovered twin waves at the receiver. This enabled demonstration of a $400 \mathrm{~Gb} / \mathrm{s}$ superchannel with a record distance of $12800 \mathrm{~km}$. This, however, is for the cost of the reduced rate by more than twice, since two polarizations were exploited carrying the same data (58).

Among approaches to compensate for nonlinearity, one can distinguish digital back propagation (59), which requires extensive signal processing at the receiver. Recently proposed digital signal processing based on the inverse scattering transform (60) offers new ways to mitigate nonlinear transmission impairments by using the ZakharovShabat spectral problem. All the proposed techniques offer effective nonlinearity compensation, though being computationally expensive.

Optical regeneration enables noise squeezing and, consequently, increases the channel capacity. In contrast to the destructive nonlinear effects, regeneration is based on the constructive use of nonlinear signal transformations which result in noise squeezing. Various schemes were offered for regenerators based on electronic or all-optical 
signal processing (61), where all-optical regeneration gained much attention recently. A number of promising results were obtained for black-box WDM (62), 4-phase-shift keying (PSK) (63)-(65), 6-PSK (66), 8-PSK (67), and 16-quadrature amplitude modulation (QAM) (68), which prove the possibility to remove noise from both quadratures of the signal. Another important feature of regeneration is cascadability, as being placed in cascades along the line regeneration enables accumulation of noise squeezing effect and, as a results, improves the received signal $(41,69,70,71)$. Thus, optical regeneration has been established as a promising and rapidly evolving area. However, though in the limits on achievable rate were estimated analytically (18) or numerically (72), proving that one can achieve transmission rate higher than the corresponding linear Shannon limit. Nevertheless, the exact regenerative capacity has not been calculated analytically, though it is highly important for analysis and design of regenerative systems.

This study addresses the aforementioned problems via an information theory framework, namely:

- we created a method for designing and optimizing regenerative channels, and derived analytical results for the regenerative capacity limit;

- we introduced a technique of regenerative Fourier transformation (RFT), which represents the continuous approximation of the ideal regenerative transfer function: the technique enables regeneration of multilevel multidimensional signal formats;

- we presented methodology for calculating the Shannon capacity of practical regenerative schemes and demonstrated it by RFT;

- we developed a procedure for simultaneous optimization of modulation format and parameters of regenerator; we demonstrated it by the example of phasequantizers based on phase-sensitive amplifiers (PSAs), for which we derived the optimal transfer function parameters and found the optimal signal packing. Moreover, we proposed a new model for multilevel amplitude regeneration coupled nonlinear optical loop mirror and demonstrated its performance on circular QAM. Also, combined with PSAs it enables simultaneous phase and amplitude regeneration for circular QAM. 
The rest of the thesis is organized as follows:

Chapter 2 provides the information theory basis with the emphasis on Shannon's seminal paper of 1948 and an overview of the previous and post-Shannon works.

Chapter 3 gives the Shannon capacity results for different communication channels: from Shannon's linear channel to currently studied fiber-optic channel.

Chapter 4 presents the results of the analysis of regenerative channels, details of the optimized regenerative mapping technique and calculations of the regenerative Shannon limit. Next, we introduced RFT technique and calculated its capacity.

Chapter 5 analyzes optimization and design of cascaded regenerative transmission. 


\section{2}

\section{Shannon capacity}

Here we give an overview of information theory and discuss the definition and meaning of the Shannon capacity.

\subsection{Introduction}

The main subject of information theory is information, a message encoded in the state of a transmitter, which is further transmitted to the receiver. By communication, we mean that the physical acts of the transmitter made influence on the physical state of the receiver. The physical process of transmitting information is subject to the noise and imperfections of the medium, signalling processes, etc. Thus, communication is perturbed by the noise, which induces errors at the receiver. Due to noise, one needs to introduce redundancy to the message to quantify that for which Shannon used entropy (1).

The main objects of information theory are ultimate data compression (defined by entropy) and the ultimate transmission rate (referred to as channel capacity, defined via the difference of entropies). Thus, information theory provides the method where, by optimizing a communication scheme over all possible input probability distributions, one can calculate the maximum achievable transmission rate, that is, the Shannon capacity. 


\subsection{Before Shannon}

The development of information theory is closely connected with telecommunication advances. Different coding techniques for information transmission can be found throughout history: from drums used for long-distance communication (with a speed of up to 100 miles in an hour) (73) to flag signalling. Starting from the first digital communication system, telegraphy, which was based on the variable-length binary code and was developed and demonstrated by Morse in 1837 (74), the technology expanded rapidly. In 1838, the five-needle telegraph was proposed by Cooke and Wheatstone. Then, in 1841, the first public telegraph from London to Birmingham was implemented with Morse's first system installed for the communication from Baltimore to Washington in 1844. In 1875, Emile Badaudot developed fixed-length binary code for telegraphy (74). The technology paved the way for efficient information coding and led to the formulation of the sampling theorem in 1924 by Nyquist $(75,76)$. Nyquist studied the maximum transmission rate by seeking an "optimum" code, which would replace Morse's for some applications. In particular, in 1939, Alec Reeves invented pulse code modulation (77). Thus started the search for efficient coding schemes, which would reduce errors due to transmission impairments. Later, Shannon formulated the problem of finding optimum input signal distribution (any combination of modulation formats and error-correcting codes).

\subsubsection{Nyquist sampling theorem}

Information theory started from the work of Nyquist $(75,76)$ in 1924 , when he investigated the problem of code optimization, which would be more efficient than the seminal code of Morse. Thus, he studied the problem of defining the maximum transmission rate in a telegraph band-limited channel in the absence of inter-symbol interference. He represented a transmitted signal in a general form:

$$
s(t)=\sum_{k} x_{k} f(t-k T)
$$

here, a waveshape is denoted by $f(t)$ and encoded binary data by $x_{k}$ transmitted at a rate $1 / T$ bits/s. This formula contains in itself the representation theorem, which 
addresses the problem of finding the minimum number of samples that uniquely determine the function $s(t)$. He showed that if a $s(t)$ has bandwidth $W \mathrm{~Hz}$, then, in the absence of inter-symbol interference, the minimum pulse rate is $2 \mathrm{~W}$ pulse/s. This rate is commonly referred to as the Nyquist rate.

The Nyquist rate under the condition of the given bandwidth $W$ can be achieved by sinc-pulses:

$$
f(t)=\frac{\sin (2 \pi W t)}{2 \pi W t}
$$

Using the above functions for shaping a signal, one can recover a transmitted information without inter-symbol interference by using samples taken at the sampling time: $k T, k=0, \pm 1, \ldots$ with $T=1 /(2 W)$. This was later generalized and proved by Shannon (1) and now is referred to as the Shannon-Nyquist theorem.

This work was further supported by a number of important results, for example, the maximum telegraph signaling speed was studied for noiseless band-limited linear signals $(75,76,78,79)$. They proved the transition rate of telegraph signals is proportional to the bandwidth of the transmission line. Earlier, independently and unbeknown to those authors, Whittaker (80)-(81) applied interpolation theory for sampling bandlimited functions.

\subsubsection{Hartley's contribution}

These results were generalized in 1928 by R. Hartley (82), who stated that, "the total amount of information which may be transmitted . . . is proportional to the product of frequency range which is transmitted and the time which is available for the transmission". In this work, Hartley used a term of "capacity" as "capacity of a system to transmit information", where he discusses that "the transmission possibilities of a complicated system ... within the range of physical possibility." Therefore, in his work, Hartley posed the fundamental problem of defining the physical limits of transmission possibilities, which he refers as "capacity". Observing RLC circuits and analyzing inter-symbol interference, he concluded that capacity is proportional to the bandwidth of the channel. Moreover, he quantized information as:

$$
H=n \log K
$$


here $K$ is the number of symbols associated with each of $n$ selections. Thus, he reflected the fundamental connection between information and selection process (randomness of the sent massage). This echoes Shannon's work where the randomness of information was presented as a selection from the chosen alphabet - modulation format. Shannon continued using the logarithmic measure of information, because he argued that it was mathematically suitable and practically useful, since transmission line parameters, such as time, bandwidth, etc., vary linearly with the logarithm of the possibilities number (this is clearly observed in the Shannon-Hartley theorem capacity of AWGN channel depends logarithmically on signal power and linearly on bandwidth). Also, Shannon, starting from Hartley's work, included noise effects and employed probabilistic methods for analyses and characterization of the communication system and for quantifying information. The probabilistic approach was also inspired by studies of cryptography, which Shannon undertook during the war and about whichhe prepared classified reports (83)-(85), for example, tables of letter frequencies for decrypting secret messages (84).

In 1946, D. Gabor (86) proposed a time-frequency uncertainty principle as a result of Heisenberg's uncertainty principles(87). The idea is similar to the one used by J. von Neumann (88) for the time-frequency shifts of a single atom. Though Gabor proposed the Gaussian functions for the signal waveform, Shannon's work gained more attention and impact on engineering.

\subsection{Shannon's pioneering work}

\subsubsection{Introduction}

Claude Shannon unified the works of his predecessors and created a unified general theory of communication in his seminal paper (1) in 1948. As Gabor noted, Shannon was also influenced by physics: he introduced entropy as a characteristic of information. This enabled him to formulate two core theorems:

1. The noiseless coding theorem, which defines the compression of information;

2. The noisy channel coding theorem, which defines the maximum reliable transmission rate over a noisy channel. 
The section will discuss both of the theorems followed by the basic notions of information theory and important results and applications of Shannon's work, which will be continued in the next chapters: the Shannon capacity of linear and nonlinear channels.

Both theorems are based on the notion of redundancy of the message, which is characterised by the entropy. The formulation of a message as Markoff process was a landmark idea. Following Hartley (82) and Wiener (89), Shannon stated that the meaning of a message is out of concern for the engineers instead: "The significant aspect is that the actual message is one selected from a set of possible messages" (1). Therefore, an information source is a physical random process, and, consequently, redundancy is a necessary part of the communication. He proposed quantifying the redundancy by the entropy. This is the key point in his theory and the two theorems address two problems:

1. redundancy of the information (information compression);

2. redundancy incorporated into a message for noise transmission.

Thus, Shannon produced a novel approach to communications: he reformulated communication systems and their components in new physical notions.

\subsubsection{Basic components of communication system}

Following Shannon, let us begin with describing a model of communication channel. Shannon distinguished the following main parts of communication system:

1. information source, creates a message - a string of symbols chosen from an alphabet. examples:

a) telegraph sequence of letters;

b) function of time in radio communications;

2. transmitter, transmitter samples, compresses, and encodes a message to produce a suitable signal suitable for transmission; examples: electric current or light

3. channel, a medium through which the signal is transmitted; 
examples: wires, optical fiber, etc.

A variety of channels and mathematical models include description of the signal distortion by noise and degradation due to nonlinearity, fading and other effects which that induce errors at at the receiver.

4. receiver,

reconstructs the message from the received signal;

5. destination,

the person or device for whom the message is intended.

Shannon represented a communication system by the basic building blocks shown in Fig. 2.1.

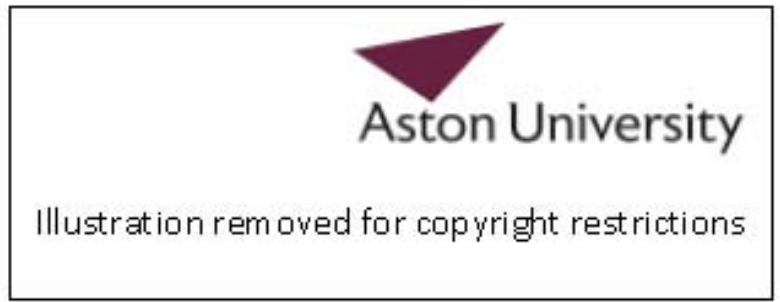

Figure 2.1: Basic components of a communication system - (taken from (1))

In this way, Shannon defined communication as a system that consists of essentially five parts listed above and designed to minimize errors during information transfer between a source and destination. The main purpose is to optimize the components to maximize efficiency. As an information source produces sequences of messages to be communicated, the distribution is subject to optimization. Then the transmitter operates on the messages to produce a signal modulated as suitable for the channel type used given the distortions that affect information. Finally, the receiver converts the signal to the form that is preferable for the destination.

To correct errors, one has to add redundancy, so the transmitted data (a set of uncoded bits) is transformed to coded bits $A_{[k]}$ by a channel encoder (see Fig. 2.2) according to the chosen coding scheme - forward error correction (FEC), e.g.for example, Reed-Solomon, or low- density parity check codes. Then a constellation mapper 


\begin{tabular}{|c|c|c|c|c|c|c|}
\hline Transmitted data & Channel & $A_{[k]}$ & Constellation & $X_{[k]}$ & Pulse & $X(t)$ \\
\hline (uncoded bits) & encoder & coded bits & mapper & symbols & shap & $\begin{array}{l}\text { signal } \\
\text { sontinus }\end{array}$ \\
\hline
\end{tabular}

\begin{tabular}{|c|c|c|c|c|c|c|c|}
\hline \multirow{3}{*}{$\begin{array}{c}\text { Fiber } \\
\text { channel }\end{array}$} & \multicolumn{4}{|c|}{ Sampling } & \multirow[b]{2}{*}{$B_{[k]}$} & \multirow{3}{*}{$\begin{array}{l}\text { Channel } \\
\text { decoder }\end{array}$} & \multirow[b]{2}{*}{ received } \\
\hline & & Filtor & & & & & \\
\hline & $Y(t)$ & & $Y_{[k]}$ & Demapper & bits & & data \\
\hline
\end{tabular}

Figure 2.2: Basic components of a communication system (expanded version) -

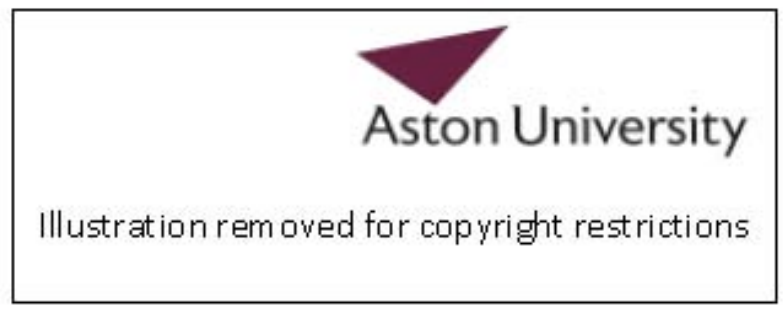

Figure 2.3: Basic modulation formats - (taken from (23)) 
assigns bits to the chosen modulation format: $A_{[k]} \rightarrow X_{[k]}$. Bit-to-symbol mapping is usually performed by channel coding techniques such as Gray coding (adjacent constellation symbols have a minimum Hamming distance of 1) or differential coding (coding in the relative phase difference between adjacent symbols). At tThe output of the mapper $X_{[k]}$ are is real or complex numbers depending on the modulation format. There are a variety of different modulation formats (see Fig. 2.2), where information is encoded in phase (phase-shift keying - PSK) or amplitude (amplitude-shift keying - ASK), a combination of amplitude and phase modulation (ASK/PSK), or quadrature amplitude modulation (QAM). In the latter symbol, points may be placed in rings (in the literature, this may be referred to as circular QAM or ASK/PSK) or on squares (in the literature, this may be referred to as rectangular or square QAM). The choice of modulation format defines an alphabet - the string of symbols, which are used for mapping. Depending on the format type, the alphabet can be real one- dimensional (e.g.for example, binary phase shift keying (BPSK) $-\{-1,1\}$, or more general amplitude shift keying $(K$-ASK) $-\{1-K, \ldots,-1,1, \ldots, K-1\})$, two-dimensional (e.g.for example, quadrature phase shift keying (QPSK) $-\{-1,1 ;-1,1\}$, which can be represented in complex numbers: $\{-1+i,-1-i, 1+i, 1-i\}$, or four-dimensional (see review in (28)). Further, the discrete-time signal $X_{[k]}$ (which can be real or complex depending on the modulation format) is transformed (by a pulse shaper) to continuoustime signals: $X(t)=\sum_{k} X_{[k]} f(t-k T)$, which is then transmitted through the media (e.g.for example, launched into the fiber channel). Nyquist pulse-shaping criterion (74) defines sinc-pulses $(\operatorname{sinc}(t-k T))$ as the optimum waveform for the bandlimited signal for no intersymbol interference (ISI). The smallest value of symbol period $T$, for which zero ISI condition holds, is defined via the signal bandwidth $T=1 / 2 / W$. However, perfect sinc-pulses are difficult to generate and in practice raised cosine pulses are usually used:

$$
f(x)=\left\{\begin{array}{lr}
T & \left(0 \leq|f| \leq \frac{1-\rho}{2 T}\right) \\
\frac{T}{2}\left(1+\cos \left[\frac{\pi T}{\rho}\left(|f|-\frac{1-\rho}{2 T}\right)\right]\right) & \left(\frac{1-\rho}{2 T} \leq|f| \leq \frac{1+\rho}{2 T}\right) \\
0 & \left(|f|>\frac{1+\rho}{2 T}\right)
\end{array}\right.
$$

This corresponds to the pulse shape:

$$
f(t)=\operatorname{sinc}(t / T) \frac{\cos (\pi \rho t / T)}{1-4 \rho^{2} t^{2} / T^{2}}, \quad t \neq 0
$$




\subsection{Shannon's pioneering work}

The parameter $\rho$ is called the roll-off factor and defines the excess bandwidth (beyond the Nyquist frequency $1 / 2 T: W=(1+\rho) / 2 T)$. For $\rho=0$ the raised cosine transforms to sinc-pulse and the excess bandwidth is 0 , for $\rho=1 / 2$ the excess bandwidth is $50 \%$, and for $\rho=1$ the excess bandwidth is $100 \%$. On the other hand, since a pulse with $\rho \neq$ 0 decays more quickly than a sinc-shaped pulse, therefore inter-symbol interference is reduced for non-perfect sampling. Apart from raised-cosine, also rectangular and Gaussian pulses are also commonly used. Recent technological advances in optical transmission have allowed creating any desirable pulse shape.

At the receiver, the transmitted continuous-time signal $Y(t)$ is converted from the optical to the electrical domain by a digital coherent receiver using analogue-to-digital conversion processes, and then the obtained digital signal is resampled at twice the symbol rate. Afterwards, digital signal processing algorithms are applied to compensate for the transmission distortions (chromatic dispersion, polarization mode dispersion, etc.) Then the signal is resampled as one sample per symbol using a clock recovery algorithm to obtain discrete-time signal representation $Y_{[k]}$ (this might also include maximum likelihood or maximum a posteriori estimation to remove degradations caused by signal transmission). Further, we denote deterministic channel input outcomes of channel input $X_{[k]}$ and output $Y_{[k]}$ by lower case $x$ and $y$ correspondingly. Afterwards, $Y_{[k]}$ is converted into bits $B_{[k]}$ by a de-mapper and then a decoder removes redundancy added due to FEC; thus, the received data is obtained. To characterize system performance, the bit error rate (BER) or symbol error rate (SER) are usually used. BER is defined as the number of bit errors (incorrectly received bits $B_{[k]}$ for given input bits $\left.A_{[k]}\right)$ per second or probability to receive incorrect bit $B_{[k]}$ for a given bit $A_{[k]}$. Similarly, SER is defined as the number of symbol errors (incorrectly received symbols $Y_{[k]}$ for given input symbols $X_{[k]}$ ) per second or probability to receive incorrect symbol $Y_{[k]}$ for a given symbol $X_{[k]}$.

Shannon classified communication systems by considering different types of sources, receivers, and channels. He included a variety of signal forms in his analysis: from simple sequences of letters used in telegraphs to more complicated forms like functions of several variables that are used in color television or radio. Thus, there are three main categories of communication systems: discrete, continuous, and mixed. The first category is related to sequences of discrete symbols: letters, dashes, dots and spaces. The most common example is Morse code that has been efficient during 


\subsection{Shannon capacity}

many decades. Another category is concerned with analog signals that are presented as continuous functions of time and other variables that characterize signal propagation.

\subsubsection{Shannon's contributions to information theory}

The framework of information and communication theory was laid by Claude Elwood Shannon in his landmark paper (1) published in 1948. In this work, he determined the basic concepts of communication systems such as the capacity and the entropy and considered different types. Above all, he included noise impact in his analysis that enabled accurate description of real systems.

By developing a new way of describing communication channels, Shannon succeeded in developing notions of the feasibility of error-free transmission, and defined entropy and mutual information (fundamental concepts applied to all communication channels). Furthermore, he defined capacity (now widely referred to as Shannon capacity) as the highest achievable error-free transmission rate and formulated channelcoding theorem, where capacity was determined as a maximum of mutual information. Thus, he laid foundations of reliable information transmission and proposed a general methodology for calculating any communication channelŠs capacity. He illustrated the concept and computed the capacity of a linear AWGN as a function of signal-tonoise ratio that is now one the most famous and important formulae in communication theory, that is, linear Shannon limit. Among his other contributions, he considered the possibility of transmitting information at a rate higher than Shannon capacity; this resulted in rate-distortion theory.

\subsection{Shannon capacity}

In this section, the Shannon capacity theorem (1) will be discussed. The theorem defines the maximum rate of reliable communication via noisy channel. It has a particular importance in information theory. 


\subsection{Shannon capacity}

\subsubsection{Entropy as a measure of information}

Representing a discrete information source as a Markoff process, Shannon used the entropy as a fundamental information measure. Shannon exploits the properties of Boltzmann entropy in statistical mechanics (90) to develop the information entropy, commonly referred to as the Shannon entropy.

Shannon defined the entropy of a discrete source with probability distribution $\left(P_{1}, \ldots, P_{K}\right)$ as

$$
H\left(\left\{x_{i}\right\}\right)=-\sum_{i=1}^{K} P_{i} \log _{2} P_{i}
$$

Thus, Shannon introduces the measure of information, choice and uncertainty. In case of two choices $(K=2$ defined by probabilities $P$ and $Q=1-P$, the entropy is equal to

$$
H\left(\left\{x_{i}\right\}\right)=-P \log _{2} P-Q \log _{2} Q
$$

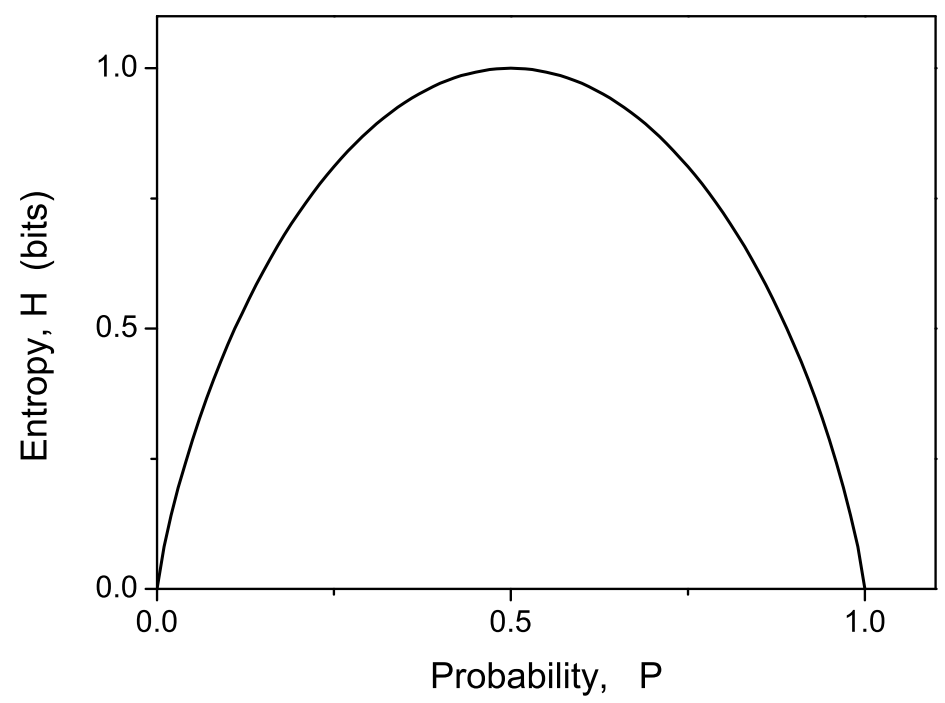

Figure 2.4: The entropy of the binary case - with probabilities $(P, 1-P)$ plotted in (1)

The entropy plotted in 2.4 demonstrates the continuous and concave dependence on the probability $P$. 


\subsection{Shannon capacity}

In comparison with Boltzmann entropy, one can list the following properties of the entropy:

1. $H(x) \geq 0$

2. For a given size of the alphabet $K$, the entropy $H$ has a maximum, equal to $\log _{2} K$, when all the selections are equiprobable, i.e. $P_{i}=1 / K$. It means that the entropy of a discrete source has maximum when the alphabet elements are equally probable.

3. The entropy of a joint event is less than or equal to the sum of the entropy of each event. The equality holds when and only when the events are independent.

4. The equalization of the probabilities increases the entropy.

In other words, the entropy increases when the probabilities are averaged:

$$
P_{i}^{\prime}=\sum_{j} a_{i j} P_{j}
$$

with coefficients positively defined $a_{i j} \geq 0$ and $\sum_{i} a_{i, j}=\sum_{j} a_{i j}=1$

5. The joint entropy is equal to the sum of one event and the conditional entropy of the other.

6. The uncertainty of one event, $y$, is never decreased by the knowledge of the other, $x$. In particular, if the events are independent, the entropy is unaffected.

$$
H(y) \geq H(y \mid x)
$$

Thus, exploiting the properties of information, Shannon defined the basic notion of information theory - the entropy. Shannon was a pioneer in borrowing the statistical methods and notions for the analysis of information. Before Shannon, Wiener introduced differential entropy (89), which was related to Fisher information of 1934 (91). The new approach enabled Shannon to develop two fundamental theorems in information theory, by which he defined the compression and transmission limits.

Shannon (1) defined the relative entropy as the ratio of the source entropy to the maximum value it could have being restricted to the same symbols, whereas the maximum compression occurs as one encodes into the same alphabet. Then, the redundancy is defined as one minus the relative entropy. Shannon demonstrated the notion of redundancy by using English. In particular, using different methods he showed that the redundancy of English is $50 \%$. 


\subsection{Shannon capacity}

\subsubsection{Mutual information}

Shannon defined the information measure - the Shannon entropy - and proved that it quantifies the number of bits required for the optimal encoding with zero losses of information. However, this is only relevant for the noiseless case, whereas if one transmits information, one can expect noise distortions, which induces errors at the receiver. Since noise is a stochastic process, the corresponding impairments, being non-deterministic effects, cannot be compensated for and information losses will be inevitable. Therefore, one has to increase redundancy to ensure successive information transmission. Consequently, to quantify information transmitted via noisy channel, where two statistical processes (the source and noise) affect the channel output, one can use mutual information $I(x, y)$.

\subsubsection{Discrete input - discrete output}

Let us consider a channel, where the constellation mapper output (see Fig. 2.2) $X_{[k]}$ is discrete symbols from a discrete alphabet $X=\left\{x_{i}\right\}, i=1 . . K$ and the output of the demapper $Y_{[k]}$ are discrete symbols: $y=\left\{y_{j}\right\}, j=1 . . K^{\prime}$. If the channel is memory-less (the output symbol $Y_{[k]}$ sampled at time $k$ is defined only by the input $X_{[k]}$ at time $k$ ) then the channel is described by the set of conditional probabilities: $P\left(y=y_{j} \mid x=x_{l}\right)$ (further $P(y \mid x)$ ).

The statistical properties of the channel are given by the conditional probability: $p(y \mid x)$, the using of which can define the output probability:

$$
P(y)=\sum_{x \in X} P(y \mid x) P(x)
$$

Using Bayes' rule, one can calculate probability distribution for the input $x$ given the received output message:

$$
P(x \mid y)=\frac{P(y \mid x) P(x)}{P(y)}
$$

Therefore, one can efficiently decode an original message knowing the received noise-corrupted $Y$ information by sending additional:

$$
H(x \mid y)=\left\langle-\log _{2} P(x \mid y)\right\rangle
$$


bits per symbol. This quantity is referred to as conditional entropy and is connected with mutual entropy as follows:

$$
H(x \mid y)=\left\langle-\log _{2} P(x, y)+\log _{2} P(y)\right\rangle=H(x, y)-H(y)
$$

and similarly

$$
\begin{gathered}
H(y \mid x)=\left\langle-\log _{2} P(y \mid x)\right\rangle= \\
=\left\langle-\log _{2} P(x, y)+\log _{2} P(x)\right\rangle=H(x, y)-H(x)
\end{gathered}
$$

These equations define the property of the conditional entropy referred to as the chain rule:

$$
H(x, y)=H(x)+H(y \mid x)
$$

Consequently,

$$
H(x)-H(x \mid y)=H(y)-H(y \mid x)
$$

The chain rule can be generalized for a number of variables: for example,

$$
H(x, y \mid z)=H(x \mid z)+H(y \mid x, z)
$$

The mutual information is measured by the information conveyed in the original message reduced by the number of additional bits due to channel impairments, therefore:

$$
I(x, y)=H(x)-H(x \mid y)
$$

or

$$
=H(x)+H(y)-H(x, y)
$$

or, similarly,

$$
=H(y)-H(y \mid x)
$$

The mutual information is symmetric under the change of variables $x$ and $y$.

In particular, if two messages are uncorrelated, then

$$
P(x, y)=P(x) P(y)
$$

which results in

$$
I(x, y)=\left\langle\log _{2} \frac{P(x, y)}{P(x) P(y)}\right\rangle=0
$$


Thus, mutual information is a measure of the amount of information that one random variable contains about another; in other words, it is the reduction in the uncertainty of one random variable due to the knowledge of the other (92). Mutual information represents the achievable rate for a given input distribution (for a given modulation format and input probabilities $P(x)$ ).

\subsubsection{Discrete input - continuous output}

Similarly, let us proceed with a channel, where the constellation mapper output (see Fig. 2.2) $X_{[k]}$ is discrete symbols from a discrete alphabet $X=\left\{x_{i}\right\}, i=1 . . K$ and the output of the demapper is unquantized (continuous variable $Y$ ). If the channel is memory-less (that is, in the absence of intersymbol interference), then the channel is described by the set of conditional pdf: $p\left(y \mid x=x_{i}\right)$. Then the conditional entropy is given as:

$$
H(y \mid x)=-\sum_{k=1}^{K} P\left(x_{k}\right) \int_{-\infty}^{\infty} d y p\left(y \mid x_{k}\right) \log _{2} p\left(y \mid x_{k}\right)
$$

and the output entropy is given as:

$$
H(y)=-\int_{-\infty}^{\infty} d y p(y) \log _{2} p(y)
$$

with

$$
p(y)=\sum_{k=1}^{K} P\left(x_{k}\right) p\left(y \mid x_{k}\right)
$$

This defines mutual information as:

$$
I(x, y)=H(y)-H(y \mid x)
$$

\subsubsection{Continuous input - continuous output}

Finally, let us proceed with a channel, where both the output and the input are continuous variables $y$. Then, the conditional entropy is given as:

$$
H(y \mid x)=-\int_{-\infty}^{\infty} \int_{-\infty}^{\infty} d x d y p(x) p(y \mid x) \log _{2} p(y \mid x)
$$

and the output entropy is given as:

$$
H(y)=-\int_{-\infty}^{\infty} d y p(y) \log _{2} p(y)
$$


with

$$
p(y)=\int_{-\infty}^{\infty} d x p(x) p(y \mid x)
$$

Mutual information is defined as:

$$
I(x, y)=H(y)-H(y \mid x)
$$

\subsubsection{Shannon capacity theorem}

Since mutual information represents the achievable rate for a specific input distribution, one can vary the latter to achieve the maximum of mutual information, which is defined as Shannon capacity - maximum achievable error-free transmission rate. As was discussed above, for efficient communication over a noisy channel, one has to increase redundancy, which is defined by the correlation between the input and output messages of the channel. Shannon showed that any stochastic channel can be used for reliable communication at non-zero rate (when the input and output are correlated) and defined the analytical expression for the optimal transmission rate. Shannon introduced the concept of the channel, as a mathematical model where noise corruption to the signal is described, and showed that the transmitter has to add redundancy.

Shannon further defined the information capacity (1) for a discrete memory-less channel as:

$$
C=\max _{p(x)} I(x, y)
$$

where optimization is over all input probability distributions $p(x)$. Capacity here is measured in bits/symbol.

Channel capacity theorem $(1,92)$ Shannon stated that "It is possible to send information at the rate $C$ through the channel with as small a frequency of errors or equivocation as desired by proper encoding. This statement is not true for any rate greater than $C^{\prime \prime}(1)$.

Thus, the theorem defines the maximum transmission rate with arbitrarily low error 


\subsection{Channel capacity and coding}

probability. It is important that the Shannon capacity is the maximum achievable reliable transmission rate. So that for all rates $R \leq C$ there are codes with maximum error probability $P_{e} \rightarrow 0$. The converse theorem states that any code with $P_{e} \rightarrow 0$ has $R \leq C$ (92).

The channel capacity theorem states that even in the presence of noise by introducing redundancy one can recover the original signal from the corrupted channel output. The proof of the theorem can be found in $(92,93)$. Note, that the Shannon capacity can be defined for continuous-time channels with specific choices of pulse shaping and filtering or discrete-time channels, whereas mutual information can be defined only for discrete-time. Further,throughout the thesis, we consider discrete-time channel models.

\subsection{Channel capacity and coding}

If the encoder maps each $k$-bit sequence into a unique $n$-bit code word (code rate $R_{c}=k / n$ ), then for a binary AWGN channel, it can be shown that the average error probability $P_{e}$ (averaged over all $k$-bit sequences) is

$$
P_{e}<2^{-n\left(R_{0}-R_{c}\right)}
$$

where $R_{0}$ is the cut-off rate:

$$
R_{0}=\log _{2}\left(\frac{2}{1+e^{-S N R}}\right)
$$

Consequently, if $R_{c}<R_{0}$, the transmission is reliable as one can achieve average error probability to be arbitrarily small when increasing the code block length $n$, since $P_{e} \rightarrow 0$ when $n \rightarrow \infty$. Thus, there are codes with probability of error less than $P_{e}$. If a code selection is random, then a probability to select a code with $P_{e}>\alpha P_{e}$ is less than $\alpha^{-1}$. On the other hand, one can reduce error probability of a selected code by increasing its dimensionality $n$.

Different cut-off rates for various equiprobable equidistant $q$-level amplitude modulation formats for an AWGN channel with average power constraint are plotted in Fig. 2.5a) alongside the upper-bound $R_{0}^{*}$, which is within $3 \mathrm{~dB}$ of the AWGN channel 
Shannon capacity (74) (see Fig. 2.5b)). There are a variety of codes that allow approaching the Shannon capacity closely, such as block codes (for example, Hamming, Hadamard, or Golay codes) or convolutional codes, which use linear finite-state shift register for encoding (see (74) for more details).

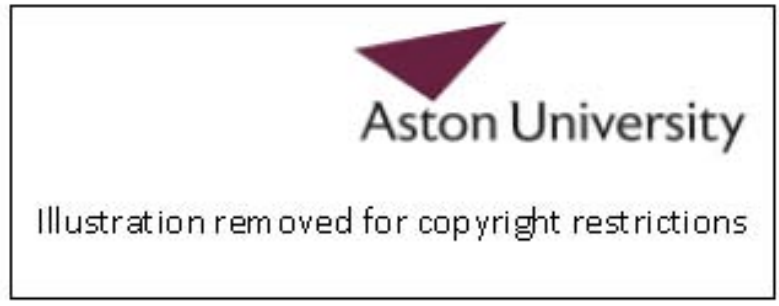

Figure 2.5: Cutoff rate (for equiprobable $q$-ASK) and capacity - for an AWGN channel with average power constraint (taken from (74))

\subsection{Communication above the Shannon capacity}

Later, in 1959 (94), Shannon continued his work by considering the problem of lossy transmission with a rate higher than the Shannon capacity. In particular, for the binary channel, Shannon calculated that with a given tolerance, bit error-rate $\varepsilon$, the maximum transmission rate is higher than the corresponding Shannon capacity by the factor (94):

$$
\frac{1}{1+\varepsilon \log _{2} \varepsilon+(1-\varepsilon) \log _{2}(1-\varepsilon)}
$$




\subsection{Communication above the Shannon capacity}

\subsubsection{Numerical calculations of Shannon capacity}

One can calculate Shannon capacity numerically for a discrete memory-less channel with fixed input and output alphabets by using the properties of Shannon capacity:

1. $C \geq 0$;

2. $C \leq \log _{2}|X|$, where $|X|$ is the size of the input alphabet;

3. $C \leq \log _{2}|y|$, where $|y|$ is the size of the output alphabet;

4. $I(X, Y)$ is a continuous function of $p(x)$;

5. $I(X, Y)$ is a concave function of $p(x)$.

Hence from the last property follows that the capacity has a unique local maximum, whereas from the properties 2 and 3 it follows that the maximum is finite for the finite alphabet; therefore, the capacity can be calculated (at least numerically). For these, one can use different methods, such as:

- Constrained maximization using the Kuhn-Tucker conditions (95), (96), generalizes the method of Lagrange multipliers for inequality constraints

- The gradient search algorithm of Frank-Wolfe (97) is an iterative first-order optimization algorithm for constrained convex optimization, also known as the conditional gradient method, reduced gradient algorithm and the convex combination algorithm. It is based on a linear approximation of the objective function in each iteration and moves slightly towards a minimum of this linear function (taken over the same domain).

- The Blahut-Arimoto algorithm (98), (99) is an iterative method that maximizes the capacity of arbitrary finite input/output alphabet sources. The optimization is performed recursively, so at each iteration:

$$
P_{k}^{(m)}=\frac{1}{Z^{(m)}} P_{k}^{(m-1)} \exp \left[H\left(P(j \mid k) \| Q_{j}^{(m-1)}\right)-S x_{k}^{2}\right]
$$

where $H\left(P(j \mid k) \| Q_{j}^{(m-1)}\right)$ denotes conditional entropy and the normalization factor is given by:

$$
Z_{k}^{(m)}=\sum_{l} P_{l}^{(m-1)} \exp \left[H\left(P(j \mid l) \| Q_{j}^{(m-1)}\right)-S x_{l}^{2}\right]
$$


The output probability distribution is:

$$
Q_{j}^{(m)}=\sum_{k} P_{k}^{(m)} P(j \mid k)
$$

Here we assumed power constraint $\sum_{k} P_{k} x_{k}^{2} \leq S$. The algorithm was modified for faster convergence using a natural-gradient-based or accelerated Blahut-Arimoto algorithm (100).

A number of algorithms were proposed for discrete alphabet (101) or continuous: using computation of a sequence of finite sums (102) or particle-based BlahutArimoto algorithm (103). In the latter, the a particles $x_{k}$ are moved to increase the relative entropy while keeping the output probability fixed.

- For discrete-input and discrete-output channels with memory an efficient technique for calculation of transmission rate was proposed (104)-(106), later generalized for continuous channels in (107).

Further, we adapted gradient search algorithm and applied Blahut-Arimoto algorithm for capacity calculations.

\subsection{Conclusion}

This chapter reviewed the fundamental theorems of information theory. The key work is Shannon's seminal paper the limits of information processing (transmission and compression) were derived. This work is described with connections to its predecessors, which led to the formulation of the Shannon-Nyquist and Shannon-Hartley theorems, as well as Shannon's successors with advances in coding and lossy compression. This is further continued in the next chapter, where various applications of Shannon capacity for different models of communication channels are discussed. 


\section{3}

\section{Shannon capacity of different communication channels}

This chapter gives an overview of different channel models and their efficiency characterized by the Shannon capacity or its associated derived lower bounds. As was demonstrated, there are a variety of linear channels with different noise distributions or band or time limitations. For most of them, the Shannon capacity was calculated. The most celebrated is the Shannon capacity of a linear AWGN channel $C=1 / 2 \log _{2}(1+S / N)$, which is commonly referred to as the linear Shannon limit. Indeed, it has become a useful reference for comparing the efficiency of different channel models or coding techniques. Nowadays, a linear AWGN channel is textbook material and wholly understood. Current progress in channel coding and processing enables approaching the linear Shannon limit (Shannon capacity of linear AWGN channel) in practical communication channels. In contrast to linear models, the Shannon capacity for nonlinear channels is an open problem. As in the linear case, there are a variety of nonlinear models; however, the Shannon capacity for most of them is not calculated. Fiber-optic channels, which are inherently nonlinear due to the Kerr effect, are responsible for over 90\% of data transmission. Though the Shannon capacity for these channels in cases of nonlinear compensation was not calculated, the achievable rate for uncompensated transmission was estimated. This result, verified experimentally and numerically, is commonly referred to as the nonlinear limit and is widely used for the prediction of the "capacity crunch". Though various methods were used to increase the channel capacity, the proposed techniques succeeded only in pushing the crunch later. However, 
existing methods enabled calculating the lower bounds, but not the Shannon capacity.

\subsection{Types of channels}

System capacity is defined by a communication channel used for information transmission. Different degradation and noise effect types, as well as equipment and practical constraints, affect channel capacity.

Among the common channel types, one can distinguish among the following (74):

1. wireline channels

examples: telephone network;

signal distortions: additive noise affects both amplitude and phase, crosstalk interference;

2. fiber-optic channels

examples: telecom and trans-oceanic communications;

signal distortions (108): the Kerr nonlinear effects (intensity-dependent refractive index), attenuation, Rayleigh and Brillouin scattering, dispersion broadening, polarization mode dispersion, modulation instability, and noise caused by amplifiers periodically placed along the transmission path to compensate attenuation during fiber transmission;

3. wireless channels

examples: sky-wave or ground-wave propagation;

distortion: noise effects, frequency absorption due to atmosphere heating, signal multipath, which causes interference fading due to destructive interference of signals arriving from different propagation paths, and multi-user interference;

4. underwater acoustic channels

distortions: attenuation of waves in water, multipath propagation (due to signal reflections from the surface and the bottom of the sea), signal fading, and noise;

5. storage channels

examples: optical and USB discs;

equivalent to above communication channels where information transmission was discussed;

distortions: noise; 
To characterize the aforementioned channels and to estimate their efficiency, mathematical models are required.

\subsection{Capacity of linear additive noise channel}

\subsubsection{Capacity of linear additive white Gaussian noise channel}

The linear Gaussian noise channel is a basic communication model that has a number of applications. In particular, it is used in radio and satellite wired and wireless communications; also fiber-optic channels can be approximated as a linear Gaussian channels in a low signal power regime.

A discrete-time linear Gaussian channel can be represented as:

$$
Y_{[i]}=X_{[i]}+\eta_{[i]}, \quad \eta_{[i]}=\mathcal{N}(0, N)
$$

where real variables are the channel output and input at the time $i$ denoted by $Y_{[i]}$ and $X_{[i]}$ (see Fig. 2.2). The input $X_{[i]}$ and the output $Y_{[i]}$ can take values $x$ and $y$ chosen from the alphabets $X$ and $y$ correspondingly. The channel is disturbed by a zero-mean additive white Gaussian noise (AWGN) $\eta_{[i]}$ with a variance $N(74)$. The additive noise is independent of the signal. The cumulative effect of a large number of small random effects, each with a well-defined expected value and well-defined variance, can be approximated by normal distribution (109).

If the noise variance is zero or there are no input constraints, the capacity is infinite. However, in practice, one has input constraints, in particular on the average input power: $\left\langle|x|^{2}\right\rangle \leq S$. The power-constrained system information capacity (1) is given by:

$$
C=\max _{\langle|x|\rangle^{2} \leq S} I(x, y)
$$

Thus, expressing the mutual information through the entropies $I(x, y)=H(y)-H(y \mid x)$ and using independence of $X$ and $\eta$, one derives the Shannon capacity per dimension of power-constrained linear AWGN channel (1):

$$
C=\max _{p(x)} I(x, y)=\frac{1}{2} \log _{2}\left(1+\frac{S}{N}\right)
$$




\subsubsection{Capacity of band-limited linear AWGN channel}

In practice, a communication channel has a limited bandwidth, which can be described by a bandpass filter. A band-limited, continuous-time channel can be represented by:

$$
Y(t)=(X(t)+\eta(t)) \star h(t)
$$

where $h(t)$ is the impulse response of an ideal bandpass filter, which limits frequencies to the bandwidth range. We assume here a white Gaussian noise denoted by $\eta(t)$.

To characterize such channels, Shannon used the results of Nyquist (75) and Gabor (86); this enabled formulating and proving the following theorem:

\section{Shannon-Nyquist sampling theorem}

Any function $f(t)$ limited in frequency to $W \mathrm{~Hz}$ (that is, the support of its Fourier spectrum is limited by $W$ ) can be completely determined by samples $f\left(\frac{n}{2 W}\right)$ taken at the Nyquist rate of $2 W$ samples per second.Taking the sum of the samples reconstructs the original time-continuous signal:

$$
f(t)=\sum_{n=-\infty}^{\infty} f\left(\frac{n}{2 W}\right) \frac{\sin [\pi(2 W t-n)]}{\pi(2 W t-n)}
$$

It is easy to check that the reconstructed function spectrum lies in the band $W$ and is zero outside. The Shannon-Nyquist theorem $(1,79,80,81)$ states that a band-limited function has $2 \mathrm{~W}$ degrees of freedom per second. The band-limited function cannot be limited in time; however, if the signal energy is concentrated in the time interval $T$, one can use expansion over prolate spheroidal functions, in particular, a number of $2 T W$; such orthogonal functions completely and uniquely define a signal (92). In a band-limited Gaussian noise channel, each sample can be considered as an independent, identically distributed Gaussian random variable. If the noise has power spectral density $N_{0} / 2$ and occupies the same bandwidth as the signal, the noise power is $N_{0} W$. Using the expression for Shannon capacity of discrete-time Gaussian channels:

$$
C_{\text {[bits per symbol] }}=\frac{1}{2} \log _{2}\left(1+\frac{S}{N}\right)
$$

Given that there are $2 W$ samples per second, one can define the following:

$$
C_{\text {[bits per second }]}=W \log _{2}\left(1+\frac{S}{N_{0} W}\right)
$$


To prove the statement, Shannon defined continuous signal entropy (1) and considered a geometrical representation of the signals (110). Thus, the formula shows the capacity as a function of signal-to-noise ratio $S N R=S / N$ and bandwidth $W$. By dividing the capacity over the band for which noise power is equal to the signal power $\left(W_{0}=S / N_{0}\right)$, Shannon (110) analyzed the bandwidth dependence of the dimensionless channel capacity: $C / W_{0}=W / W_{0} \log _{2}\left(1+S /\left(N_{0} W\right)\right)=W / W_{0} \log _{2}\left(1+W_{0} / W\right)$ (see Fig. 3.1), which in the limit $W \rightarrow \infty$ tends to $C / W_{0} \rightarrow \log _{2}(e)$. If one will consider $W \rightarrow \infty$, the capacity will tend to $C \rightarrow S / N$. Hence, for infinite bandwidth, capacity increases linearly to infinity as a function of signal power. There are a number of channel codes that enable approaching the Shannon linear limit; see (84).

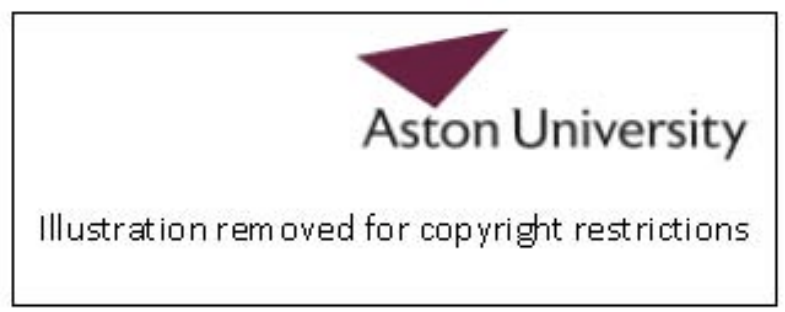

Figure 3.1: Dependence of the Shannon capacity of the linear AWGN channel on channel bandwidth - (here, signal power was denoted by $P$ ) (taken from (110))

\subsection{Nonlinear fiber-optic channel}

\subsubsection{Model}

The communication channel in which a fiber-optic cable is used as a transmission medium is significantly nonlinear due to the refractive index dependence on the signal power or due to scattering phenomena (108). As the scattering phenomena associated with Brillouin and Raman effects can be reduced by controlling the signal power, 
whereas refractive index dependence on the signal power, referred to as the Kerr effect (observed in 1875 by John Kerr (111)) causes severe degradation to the signal and limits the channel capacity.

The propagation of an electric field $\mathbf{E}$ is governed by (112):

$$
\nabla^{2} \mathbf{E}-\frac{1}{c^{2}} \frac{\partial^{2} \mathbf{E}}{\partial t^{2}}=-\mu_{0} \frac{\partial^{2} \mathbf{P}(\mathbf{E})}{\partial t^{2}}
$$

where $c$ is the speed of light and $\mu_{0}$ is the vacuum permeability.

In case of weak nonlinearity, one can expand the polarization vector $\mathbf{P}$ as follows:

$$
\mathbf{P} \approx \varepsilon_{0}\left(\chi^{(1)} \mathbf{E}+\chi^{(2)}: \mathbf{E E}+\chi^{(3)}: \mathbf{E E E}\right)
$$

here $\varepsilon_{0}$ is the electric permittivity of free space, : denotes the inner tensor product, whereas $\chi^{(1)}$ is the linear susceptibility, and $\chi^{(2)}$ governs the second harmonic generation, whereas $\chi^{(3)}$ defines the third harmonic generation and Kerr effect. Further, one can omit $\chi^{(2)}$ for silica fibers (as $\mathrm{SiO}_{2}$ is a symmetric molecule). The Kerr nonlinearity is responsible for: phase shift acquired by signal propagation - self-phase modulation (SPM), phase shift due to the influence of signals having different carrier frequencies cross-phase modulation (XPM), four-wave mixing (FWM) - interaction between signals whose frequencies satisfy the phase matching conditions, the interplay between chromatic dispersion and Kerr effect - modulation instability.

The scalar evolution of linearly polarized along the fiber-optic field in the presence of Kerr effect in a single-mode fiber with slowly varying envelope and rotating wave approximations (after separation of rapidly varied part around the carrier frequency $\omega_{0}: \mathbf{E}=2^{-1} \mathbf{e}_{x} E(z, t) \exp \left(-i \omega_{0} t\right)+$ c.c, where $\mathbf{e}_{x}$ is a unit vector in $x$-axis $)$, is given by the nonlinear Schrödinger equation (NLSE) (113) :

$$
\frac{\partial E}{\partial z}+\beta_{1} \frac{\partial E}{\partial t}+i \frac{\beta_{2}}{2} \frac{\partial^{2} E}{\partial t^{2}}+\frac{\alpha}{2} E=i \gamma E|E|^{2}+\frac{\beta_{3}}{6} \frac{\partial^{3} E}{\partial t^{3}}-a_{1} \frac{\partial\left(E|E|^{2}\right)}{\partial t}-a_{2} E \frac{\partial|E|^{2}}{\partial t}+\eta(t, z),
$$

here dispersion coefficients are given in a Taylor series of the propagation constant $\beta(\omega)$ around the carrier frequency $\omega_{0}$ :

$$
\beta_{j}=\left.\frac{d^{j} \beta(\omega)}{d \omega^{j}}\right|_{\omega_{0}}
$$


Hence, the cubic and higher order terms can be neglected under the condition that the spectral width of the pulse satisfies the condition $\Delta \omega \ll \omega_{0}$. The nonlinear parameter is defined as:

$$
\gamma\left(\omega_{0}\right)=\frac{\omega_{0} n_{2}}{c A_{e f f}}
$$

where

$$
n_{2}=\frac{3}{4} \frac{\operatorname{Re}\left(\chi_{x x x x}^{(3)}\right)}{\varepsilon_{0} n^{2} c}
$$

is the fiber nonlinear refractive index ( $n$ is a linear part of a fiber refractive index $\tilde{n}=n+\tilde{n}_{2}|E|^{2}$ ) and $A_{e f f}$ is the fiber effective area (108). The parameter $\alpha$ denotes the linear part of a fiber attenuation coefficient. The Langevin-noise source term $\eta$ incorporates the stochastic perturbations of the signal. The NLSE is valid if the signal polarization does not vary along the fiber length (it was experimentally demonstrated that the optical field remains co-polarized in long-haul transmission over thousands of $\mathrm{km}(113,114))$ and the optical field is quasi-monochromatic: the spectral width of the spectrum satisfies $\Delta \omega / \omega_{0} \ll 1$. In particular, since $\omega_{0} \approx 10^{15} s^{-1}$, then pulse width is of the order of $0.1 \mathrm{ps}(108)$.

The term $a_{1}$, which describes the self-steepening effect of the pulse edge, can be neglected in optical communications. Also, the term $a_{2}$, which characterizes the Raman effect, can be reduced by limiting the signal power and bandwidth $(108,113)$. Therefore, the NLSE can be simplified as

$$
\frac{\partial E}{\partial z}+\beta_{1} \frac{\partial E}{\partial t}+i \frac{\beta_{2}}{2} \frac{\partial^{2} E}{\partial t^{2}}+\frac{\alpha}{2} E=i \gamma E|E|^{2}+\eta(t, z)
$$

or after a change of variables: $\tau \rightarrow t-\beta_{1} z$ :

$$
\frac{\partial E}{\partial z}+i \frac{\beta_{2}}{2} \frac{\partial^{2} E}{\partial \tau^{2}}+\frac{\alpha}{2} E=i \gamma E|E|^{2}+\eta(\tau, z)
$$

Thus, the resulting equation includes deterministic, as well as stochastic, linear (dispersion and attenuation) and nonlinear Kerr effects.

In standard, single-mode fiber, the attenuation coefficient is $0.2 \mathrm{~dB} / \mathrm{km}$ in the 1550 $\mathrm{nm}$ wavelength region (23); consequently, optical amplification is required (in the absence of opto-electronic regeneration). This induces noise, depending on amplification scheme: amplified spontaneous emission (ASE) or double Rayleigh scattering (23). As spontaneous emission, the result of a photon due to the transition from an excited 
to the ground state $(115,116)$ is a random process; the associated quantum-mechanical fluctuations are referred as ASE. In 1963, Gordon $(117,118)$ showed that ASE can be represented as a circularly symmetric additive Gaussian noise, which is fully defined by the autocorrelation function $(23,108)$ :

$$
\left\langle\eta(\tau, z), \eta^{*}\left(\tau^{\prime}, z^{\prime}\right)\right\rangle=N_{A S E} / L \delta\left(z-z^{\prime}\right) \delta\left(\tau-\tau^{\prime}\right)
$$

Here, $\delta$ functions ensure that the noise realizations in time and space are independent of each other. Here $N_{A S E}$ is the power spectral density of the ASE noise, accumulated during the signal propagation over the distance $L$.

The vector NLSE with account of polarization effects and with assumption of low values of polarization-mode dispersion (PMD) and slow varied birefringence with averaging the nonlinear terms over birefringence fluctuations was derived by Manakov (119) and subsequently verified in $(120,121)$ :

$$
\begin{aligned}
& \frac{\partial E_{1}}{\partial z}=-\frac{\alpha}{2} E_{1}-i \frac{\beta_{2}}{2} \frac{\partial^{2} E_{1}}{\partial t^{2}}+i \frac{8 \gamma}{9}\left(\left|E_{1}\right|^{2}+\left|E_{2}\right|^{2}\right) E_{1}+\eta_{1}, \\
& \frac{\partial E_{2}}{\partial z}=-\frac{\alpha}{2} E_{2}-i \frac{\beta_{2}}{2} \frac{\partial^{2} E_{2}}{\partial t^{2}}+i \frac{8 \gamma}{9}\left(\left|E_{1}\right|^{2}+\left|E_{2}\right|^{2}\right) E_{2}+\eta_{2}
\end{aligned}
$$

here indexes denote two polarizations for electric field $E_{1,2}$ and noise $\eta_{1,2}$.

The basic equation 3.15 apart from being the principal master model, can also be directly derived in two practically important periodic amplification schemes: (i) periodically spaced discrete point amplifiers (for example, Erbium-doped fiber amplifiers), and (ii) quasi-lossless distributed Raman amplification where the Raman gain continuously compensates for the fiber loss and signal average power remains constant along the entire transmission span

In particular, if losses are ideally compensated (that is, $\alpha=0$ in Eq. 3.15) by periodically inserted amplifiers (for example, Erbium-doped fiber amplifiers (EDFAs) $(108,122)$, or if the amplifier spacing is much smaller compared with the length scale of nonlinear effects $(\gamma S)^{-1}$ or is continuous quasi-lossless distributed over the transmission path Raman amplification $(15,123,124)$, the path-averaged model can be applied leading to the following equation:

$$
\frac{\partial E}{\partial z}+i \frac{\beta_{2}}{2} \frac{\partial^{2} E}{\partial t^{2}}=i \gamma E|E|^{2}+\eta(t, z),
$$


The NLSE and Manakov equations characterize the signal propagation along the fiber and include all aforementioned degradation phenomena. In particular, if SPM and XPM are of equal strength and losses are ideally compensated over entire transmission path, the nonlinear equations are integrable by the inverse scattering method (125, 126).

\subsubsection{Simplified channel models}

The above-presented propagation equations (NLSE and Manakov system) describe complex nonlinear continuous-time communication channels. Corresponding conditional discrete-time probabilities are not known in the general case for these channels. Now we consider simplified models with limited applicability, in which capacity can be estimated or computed. The continuous-time model can be transformed into a discretetime model by expanding the continuous-time signal (complex input $X(t)$ and output $Y(t)$ ) over a complete set of orthogonal functions (74). This results in discrete-time complex variables $X_{[k]}$ and $Y_{[k]}$ (see Fig. 2.2). Further, we omit indexes for memoryless channels, except MGM channel in Sec. 3.3.2.6. Input $X$ and output $Y$ can take values $x=r_{\text {in }} e^{i \varphi_{\text {in }}}$ and $y=r_{\text {out }} e^{i \varphi_{\text {out }}}$ chosen from the alphabets $\mathrm{X}$ and $\mathrm{Y}$ correspondingly.

\subsubsection{Constant-intensity modulation with coherent detection}

Channel using constant-intensity modulation formats with coherent detection is a linear AWGN channel $(127,128,129)$ :

$$
Y=X+\eta
$$

with complex $x$ and $y$ and additional constraint $|x|^{2}=S$

$$
p\left(y \mid x=\sqrt{S} e^{i \varphi_{i n}}\right)=\frac{1}{\pi N} \exp \left[-\frac{\left|y-\sqrt{S} e^{i \varphi_{i n}}\right|^{2}}{N}\right]
$$

In (127) Shannon, capacity was calculated numerically and the asymptotic expression was found for high SNR:

$$
C \rightarrow \frac{1}{2} \log _{2}\left(\frac{4 \pi}{e} S N R\right)
$$




\subsubsection{Intensity-modulated direct-detection (IMDD) channel}

The simplest communication systems are intensity-modulated direct detection (IMDD) $(2,3)$, where signal power is modulated and detected. A photo-detector responds to the square root of the incident electric field; thus, the output and input are connected as follows:

$$
Y=|X+\eta|
$$

The conditional pdf for this model was found to be the Rice distribution (2):

$$
p(y \mid x)=\frac{|y|}{\pi N} \exp \left(-\frac{|y|^{2}+|x|^{2}}{N}\right)
$$

where $I_{0}$ is a zero-order modified Bessel function of the first kind. It was shown in $(2,3)$ that the capacity-achieving distribution has a number of infinite mass points; also, lower and upper bounds on capacity were presented, including the asymptotically tight lower bound (130, 131):

$$
C_{u b}^{I M D D}=\frac{1}{2} \log _{2}(1+S N R)-0.5
$$

Thus, the Shannon capacity of the IMDD channel is a monotonically increasing function of SNR and the capacity-achieving distribution has an infinite number of mass points and does not contain an infinite set of mass points on any bounded interval (3).

\subsubsection{Partially coherent AWGN}

The discrete-time partially coherent (phase detection is applied) AWGN channel (132, 133) can be introduced as:

$$
Y=X e^{i \Theta}+\eta
$$

Here, the additive noise $\eta$ is above a complex Gaussian random variable with zero mean. Variable $\Theta$ mimics the phase noise and can be modelled as real-valued wrapped AWGN with variance $\sigma_{\Phi}^{2}$ as in $(132,133)$.

The conditional pdf for such channels can also be found:

$$
p(y \mid x)=\int_{-\pi}^{\pi} \frac{d \theta}{\pi N} \exp \left[-\frac{\left|y-x e^{i \theta}\right|^{2}}{N}\right] \frac{1}{\sqrt{2 \pi \sigma_{\Phi}^{2}}} \sum_{k=-\infty}^{\infty} \exp \left[\frac{(\theta-2 \pi k)^{2}}{2 \sigma_{\Phi}^{2}}\right]
$$


If a phase tracking device (such as phase-locked loop) is employed, than $\Theta$ might be modelled by Thikhonov distribution (3) with positive parameter $\rho$ :

$$
p(\theta)=\frac{e^{\rho \cos (\theta)}}{2 \pi \mathrm{I}_{0}(\rho)} 1
$$

The conditional pdf for such channels was derived as:

$$
\begin{gathered}
p(y \mid x)=\frac{r_{\text {out }}}{\pi N} \exp \left[-\frac{r_{\text {out }}^{2}+r_{\text {in }}^{2}}{N}\right] \frac{\mathrm{I}_{0}(\mathrm{v})}{\mathrm{I}_{0}(\rho)} \\
v=\sqrt{4 \frac{r_{\text {out }}^{2} r_{\text {in }}^{2}}{N^{2}}+4 \rho \frac{r_{\text {out }} r_{\text {in }}}{N} \cos \left(\varphi_{\text {in }}-\varphi_{\text {out }}\right)+\rho^{2}}
\end{gathered}
$$

The capacity-achieving distribution was shown to be circularly symmetric (4) and (similar to the result in the previous subsection) does not contain an infinite set of mass points on any bounded interval (3).

In the case when $\Theta(x)$ is not a random variable (134), the conditional pdf is simplified to:

$$
p(y \mid x)=\frac{1}{\pi N} \exp \left[-\frac{\left|y-x e^{i \theta(x)}\right|^{2}}{N}\right]
$$

In (134), it was demonstrated that estimation based on Gaussian approximation deviates from the exact Shannon capacity of the channel given by $W \log _{2}(1+S N R)$ with capacity-achieving distribution:

$$
p(x)=\frac{1}{\pi S}\left[1+i \gamma\left(x^{*} \frac{\partial \theta(x)}{\partial x^{*}}-x \frac{\partial \theta(x)}{\partial x}\right)\right] \exp \left[-\frac{|x|^{2}}{S}\right]
$$

\subsubsection{Nonlinear phase noise channel}

In a phase noise channel $(17,135,136,137,138)$, the output is given via the input as:

$$
Y=(X+\eta) e^{-i \Phi_{N L}}
$$

here, $\eta$ is the total AWGN accumulated on the transmission path. The equation models NLSE Eq. 3.15 with zero dispersion $\beta_{2}=0$ and periodically inserted ASE and describes the phase noise caused by the SPM effect:

$$
\frac{d E(z)}{d z}=-i|E(z)|^{2} E(z)+\eta,\left\langle\eta(z), \eta^{*}\left(z^{\prime}\right)\right\rangle=\sigma^{2}\left(z-z^{\prime}\right)
$$


The nonlinear phase noise is given as (135):

$$
\Phi_{N L}=\frac{\gamma L}{N_{s}} \sum_{i=1}^{N_{s}}\left|X+\eta_{i}\right|^{2}
$$

Here, summation is performed over the ASE noise accumulated at each of $i=1 . . N_{s}$ fiber segments: $\eta_{i}=\zeta_{1}+\ldots+\zeta_{i}\left(\zeta_{i}\right.$ denotes ASE noise aroused at $i$-th span). The conditional pdf for such channel (after proper normalization described in $(17,135$, 138)) reads:

$$
p\left(y=r_{\text {out }} e^{i \varphi_{\text {out }}} \mid x=r_{\text {in }} e^{i \varphi_{\text {in }}}\right)=\frac{p\left(r_{\text {out }} \mid r_{\text {in }}\right)}{2 \pi}+\frac{1}{\pi} \sum_{m=1}^{\infty} \operatorname{Re}\left(e^{i m\left(\varphi_{\text {out }}-\varphi_{\text {out }}\right)} K_{m}\left(r_{\text {out }}, r_{\text {in }}\right)\right)
$$

where $p\left(r_{\text {out }} \mid r_{\text {in }}\right)$ is the Ricean pdf of the received power:

$$
p\left(r_{\text {out }} \mid r_{\text {in }}\right)=\frac{2 r_{\text {out }}}{N} \exp \left(-\frac{r_{\text {out }}^{2}+r_{\text {in }}^{2}}{N}\right) \mathrm{I}_{0}\left(\frac{2 r_{\text {out }} r_{\text {in }}}{N}\right)
$$

and the analytical expressions for the coefficients $C_{m}\left(r_{\text {out }}, r_{\text {in }}\right)$ :

$$
\begin{gathered}
K_{m}(r)=r_{\text {out }} b_{m} \exp \left[-a_{m}\left(r_{\text {out }}^{2}+r_{\text {in }}^{2}\right)\right] I_{m}\left(2 b_{m} r_{\text {out }} r_{\text {in }}\right) \\
a_{m}=\frac{\sqrt{i m \gamma}}{\sigma} \operatorname{coth} \sqrt{i m \gamma \sigma^{2}} z \\
b_{m}=\frac{\sqrt{i m \gamma}}{\sigma} \frac{1}{\sinh \sqrt{i m \gamma \sigma^{2}} z}
\end{gathered}
$$

here $\mathrm{I}_{m}$ is the modified Bessel function.

\subsubsection{Optical fiber channels with zero average dispersion}

The NLSE can be rewritten in case of zero average dispersion for a complex function $Y(z)=E(z, 0)(17)$ :

$$
\frac{d Y}{d z}=-i|Y|^{2} Y+\eta
$$

with zero-mean AWGN noise having variance $\left\langle\eta^{*}\left(z^{\prime}\right) \eta(z)\right\rangle=D \delta\left(z-z^{\prime}\right)$, where $D$ is the regularized noise intensity. The channel conditional probability:

$$
p(y \mid x)=\sum_{m=-\infty}^{\infty} \frac{e^{i m\left(\varphi_{\text {out }}-\varphi_{\text {out }}\right)}}{\pi D} r e^{-\frac{r_{\text {in }}^{2}+r_{\text {out }}^{2}}{D} k_{m} \operatorname{coth} k_{m} z} \frac{k_{m}}{\sinh k_{m} z} I_{|m|}\left(\frac{2 k_{m} r_{\text {out }} r_{\text {in }}}{D \sinh k_{m} z}\right),
$$


where $k_{m}=\sqrt{i m D}$ and $I_{m}$ is the modified Bessel function. The impact of optimization of symbol error probability over amplitude phase-shift keying constellations was studied in $(135,136,137)$. The lower bound on channel capacity was derived in (17):

$$
C \geq \frac{1}{2} \log _{2}(S N R)
$$

\subsubsection{Infinite-memory Gaussian noise channel model}

The general channel model NLSE can be rewritten in the case of wavelength-division multiplexing (WDM) in the form that shows evolution of signal in each WDM channel (the optical field of $k$-th channel) with account of SPM and XPM effects (139):

$$
\frac{\partial E_{k}}{\partial z}=-i \frac{\beta_{2}}{2} \frac{\partial^{2} E_{k}}{\partial t^{2}}+i \gamma E_{k}\left(\left|E_{k}\right|^{2}+\sum_{j \neq k} 2\left|E_{j}\right|^{2}\right)+\eta(t, z),
$$

Here, $\beta_{2}$ and $\gamma$ denote dispersion and nonlinearity coefficients and ideal compensation of fiber losses is assumed, with $\eta(t, z)$ being aspontaneous emission noise, which results from the signal amplification during propagation. This presentation allows introducing another simplified channel model by omitting intra-channel effects. This may be justified in the regime when the inter-channel effects are dominant nonlinear effects or when self-phase modulation is compensated (140)-(142)). In this case, the resulting model equation has the form:

$$
\frac{\partial E_{k}}{\partial z}=-i \frac{\beta_{2}}{2} \frac{\partial^{2} E_{k}}{\partial^{2} t}+i \gamma E_{k} V_{k}+\eta(t, z),
$$

where the effective potential $V_{k}$ is

$$
V_{k}=\sum_{j \neq k} 2\left|E_{j}\right|^{2}
$$

In dispersion uncompensated transmission, the potential $V_{k}$ can be modelled (approximately) as a Gaussian stochastic process short-range correlated in space and time. This leads to the following simplified channel model with multiplicative Gaussian noise:

$$
Y_{i}=A X_{i}+B H_{i j} X_{j}+\eta
$$

where the output spectrum is denoted as $y_{i}=\tilde{E}_{S}\left(\omega_{i}, z\right)$, whereas the input signal is $x_{i}=\tilde{E}_{s}\left(\omega_{i}, 0\right)$. The inter-channel interference is given by the matrix $H_{i j}$, which is 
assumed to be a zero-mean, Gaussian-independent and identically distributed complex random matrix with variance $<H_{i k} H_{j l}>=m^{-1} \delta_{i j} \delta_{k l}$, here $m$ is dimensionality of the signal. Factor $B$ defines the strength of signal-signal interactions. The conditional pdf of such a model is given in (13):

$$
p(y \mid x)=\frac{1}{\left(2 \pi \sigma^{2}\right)^{m}} e^{-|y-A x|^{2} / \sigma^{2}}
$$

where variance is given by:

$$
\sigma=m^{-1}\left(\left\langle|\eta|^{2}\right\rangle+B^{2}|x|^{2}\right)
$$

Various authors have followed a similar generic approach, which shares the same assumptions, but includes a variety of different physical effects. These produce slightly different lower bounds on capacity (including impairments from SPM, XPM, and FWM effects), which can be generalized by the following formula:

$$
C_{L B}=W \log _{2}\left(1+\frac{S}{N+\mu S^{3}+v S^{2} N}\right) \quad \text { bits } / \mathrm{s}
$$

This summarizes the results for a variety of communication channels, such as OFDM or WDM, with arbitrary dispersion, distributed or lumped amplification, for each of which the coefficients are found considering different nonlinearities: $\mu$ reflects signalsignal interactions, such as FWM and XPM $((7,11,14,18,23,47))$ and $v$ arises from signal-noise interactions $((143,144))$.

\subsection{Conclusion}

This chapter gives an overview of analytical modelling of different communication channels in the context of the Shannon channel capacity. The most important and challenging limit on fiber channel spectral efficiency is nonlinearity. The increase in the signal power leads to power-dependent nonlinear transmission distortions. There is a clear challenge to develop radically new approaches to coding, transmission, and processing of information in fiber communication channels. Next the Shannon capacity of regenerative nonlinear channels is presented. 


\section{4 \\ Shannon capacity of nonlinear regenerative channels}

\subsection{Introduction}

There is a growing interest in studies of capacity for regenerative nonlinear communication channels, which are principally different from decode-and-forward channels, as the term "regenerator" is used here in the context of a device (noise squeezer) that has a smooth nonlinear regenerative transfer function without any decision making. For such channels, the capacity limits have yet to be defined. The progress in experimental demonstration of all-optical signal regeneration (145)-(148), including phase regeneration, paves the way to new possibilities in the constructive use of nonlinearity.

Regeneration may take many forms, ranging from decode-and-forward (allowing the use of FEC in each stage), hard decision (without FEC) (149), transformation with smooth nonlinear transfer functions (TF), which results in noise squeezing (61), and distributed pulse and noise shaping (150). System capacities for the decode-andforward model can be readily calculated from the capacity of a single link channel (151), while another approach is required for hard decision and smooth TF-based regenerators, which can be realized all-optically.

Recent studies have proved (72) that regenerative channels have Shannon capacity higher than systems without regenerators operating with the same signal launch power and with the same noise per amplifier. Recent advances opened regeneration for coherent communication, for example, multilevel phase regeneration $(63,152)$. 
Nevertheless, all-optical regeneration faces a number of challenges as it still requires full dispersion compensation and has a limited capability for multi-wavelength performance. Although a number of works were proposed for multichannel regeneration (for the case of coherentcommunication, see (65)), these are only for a limited number of channels and the issue remains open. Furthermore, new schemes are required to suppress phase and amplitude noise for various multilevel modulation formats and for different pulse shapes.

In this chapter, we estimate the benefits of regeneration implementation in the bestcase scenario - Shannon capacity for fully optimized 3R regenerators, the best transmission performance due to removal of timing jitter effects, and optimized nonlinear response by regenerative mapping. In the next chapter, we propose new models for simultaneous suppression of phase and amplitude noise for different circular and rectangular multilevel modulation formats.

Here, we present the first calculation of the regenerative capacity benefit with system optimization, and we propose the optimization technique for the regenerative element - regenerative mapping and demonstrate it on the new model - regenerative Fourier transform. The proposed transformation represents the first terms in the Fourier expansion and, therefore, is a close approximation of the ideal regenerator. By calculating an analytic result for the Shannon capacity gain due to regeneration with simultaneous optimization of signal modulation and regenerative characteristics, we prove the Shannon capacity improvement above the corresponding linear additive white Gaussian noise channel without requiring decoder/encoder pairs to be used with in-line elements. Finally, we quantify the Shannon capacity of the ideal regenerator, which defines the highest efficiency - regeneration limit.

\subsection{Regenerative channel}

Consider a nonlinear regenerative channel with $R$ identical nonlinear regenerators placed along the transmission line. The signal transmission is distorted by a stochastic process that is modelled as AWGN uniformly distributed along the line. The noise term incorporates the stochastic effects from different sources. Depending on the model application, the stochastic process can be considered analogous to the random force in the time-continuous case of noise mixing with the signal during transmission. This 
term also includes an additive noise that originates from the nonlinear device itself. We place regenerative elements equidistantly along the transmission line.

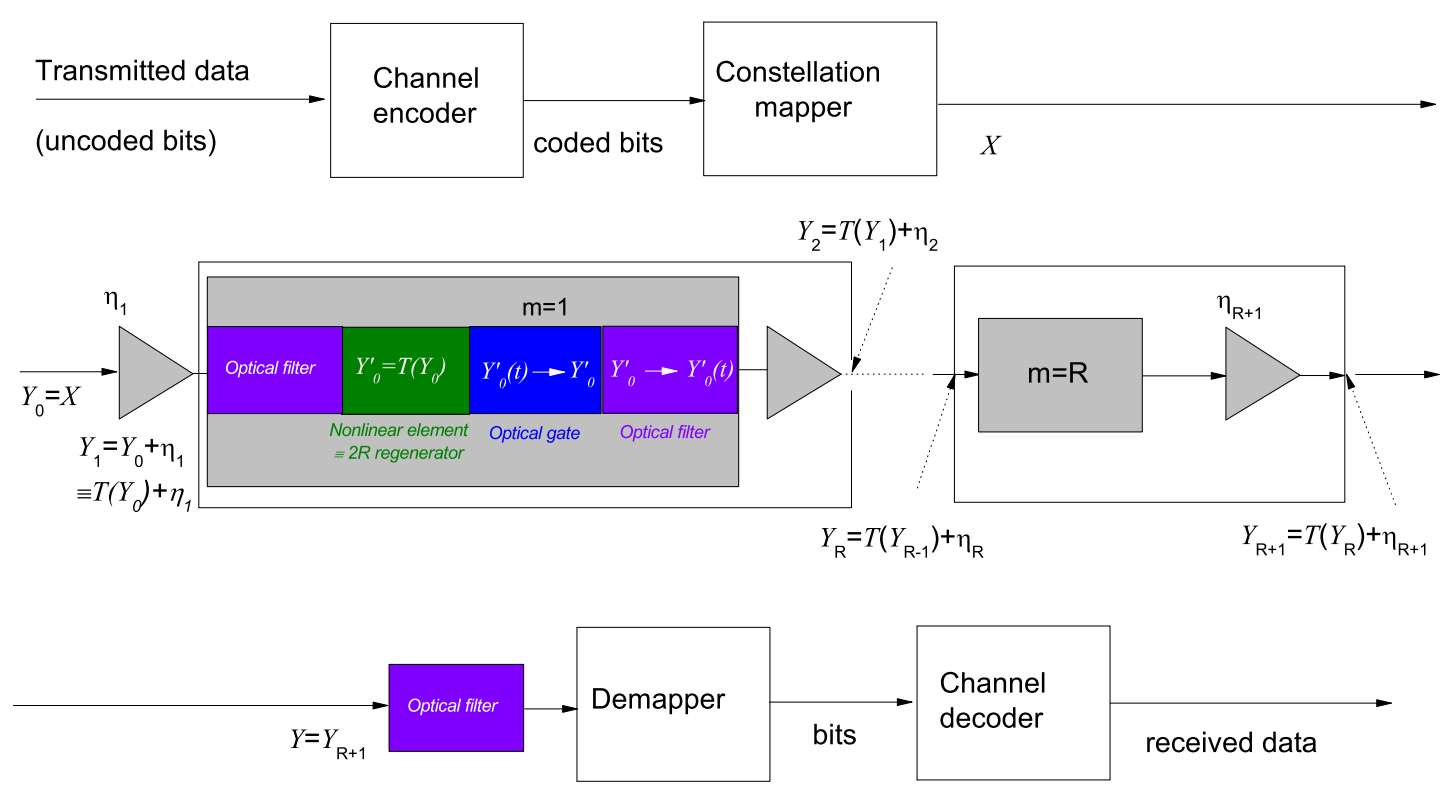

Figure 4.1: The regenerative channel model -

To maximize the Shannon capacity of regenerative channels, one needs $3 R$ regenerators, which allows suppressing timing jitter phenomena. The architecture of such elements is depicted in Fig. 4.1 and consists of three stages. At the first stage, an optical filter (here and further, a matched filter to rectangular pulses) is used to maximize the output signal-to-noise ratio; then, a nonlinear regenerative transformation is applied to a noise- distorted signal: $Y_{m+1}(t)=T\left[Y_{m}(t)\right]+\eta_{m+1}(t)$. This is followed by an optical gate, which samples the point of a minimum amplitude noise so that a continuoustime signal at the link $m, Y_{m}(t)$, is transformed to its discrete-time form $Y_{m}$, which is then shaped by an optical filter. Thus, the discrete-time signal is converted again to its continuous-time form, to receive a regenerated signal with squeezed amplitude and timing distortions. Here we assume that a regenerator handles both signal quadratures of complex $Y_{m}$ independently: $\operatorname{Re}\left(Y_{m}^{\prime}\right)=T\left[\operatorname{Re}\left(Y_{m}\right)\right]$ and $\operatorname{Im}\left(Y_{m}^{\prime}\right)=T\left[\operatorname{Im}\left(Y_{m}\right)\right]$. This ensures best regenerative efficiency as there is no signal-signal interference. Thus, the two-dimensional problem (with complex $Y_{m}$ ) can be reduced to the 2 independent one-dimensional lattices. Further, for simplicity, we omit Re, Im notations and use $Y_{m}$ 
for one-dimensional real discrete-time signal at $m$-th link. After addition of noise, the signal is: $Y_{m+1}(t)=Y_{m}^{\prime}(t)+\eta_{m+1}(t)$. Expanding the noise over the appropriate set of orthonormal functions, we can consider the continuous-time problem in its discretetime form: $Y_{m+1}=Y_{m}^{\prime}+\eta_{m}=T\left(Y_{m}\right)+\eta_{m+1}$. Further, we use $Y_{m}$ for one-dimensional real discrete-time signal output at $m$-th link and lower case $y_{m}$ for its deterministic outcome. Similarly, $x_{k}$ notates real $k$-th alphabet point (that is, in the QPSK case, the modulation can be considered as two one-dimensional lattices with $x_{k}=\{-1,1\}$ ).

\subsubsection{Numerical verification}

It is important that the validity of the model depends on the time window of an optical gate. For continuous-time modelling, we simulated numerically $5 \cdot 10^{5}$ of equiprobable symbols modulated as 16-QAM format shaped as ideal rectangular pulses with a baud rate of $B=28$ GBaud. A nonlinear transformation was applied to an optical continuous-time signal: $T(y)=y+\pi^{-1} \sin (\pi y)$. The optical filter used was matched for rectangular pulses. The duration of the optical gate time window was varied. An operating point of the regenerator has been optimized, so that there is no spectral broadening along the line; in other words, pulses maintain fixed duration during transmission. An eye-diagram, waveform, and spectrum of the output of the 20th regenerator are plotted in Fig. 4.2 for noiseless transmission and Fig. 4.3 noisy (the corresponding $O S N R=10 \mathrm{~dB}$ ) transmission. The signals were normalized by the input signal power. It is seen that the $3 \mathrm{R}$ regenerator does not affect the undistorted signal (see Fig. 4.2) and the waveform is conserved. Figure 4.3 demonstrates that noise is effectively suppressed and signal transmission is significantly improved. Figure 4.4 shows noise suppression for $R=5 \mathrm{in}$-line regenerators with the same level of $O S N R=10 \mathrm{~dB}$. Note that the regenerator acts as a noise squeezer, so that it reduces distortion effects without signal amplification - signal power is not increased. The power ratio for the output signal of the $R$-th regenerator to theinput signal is plotted in panel d of Figs.4.2-4.4 for $R=20$ and $R=5$ for noiseless and noisy transmission.

Also, we calculated the symbol error rate (SER) by direct error counting using Monte Carlo simulations as a function of optical SNR (OSNR) $O S N R=S N R \frac{B}{2 B_{r e f}}$, where $B_{r e f}=12.5 \mathrm{GHz}$. SNR is defined here as the ratio of the input signal power $S$ to the noise (zero mean AWGN with variance $N_{m}$ ) added linearly to the signal during 


\subsection{Regenerative channel}

transmission at each node $m=1, \ldots, R+1: S N R=S / N$, where accumulated noise is given by $N=\sum N_{m}$, where $N_{m}$ is noise power added after transmission via $m-t h$ node. Note, in the nonlinear communication system, due to the mixing of signal with noise during propagation, the definition of in-line SNR is a non-trivial issue. The introduced SNR occurs in the respective linear system in the absence of nonlinear in-line elements. This allows us to compare the considered system with the corresponding linear AWGN channel having the same noise level. Evidently, the effect of noise squeezing is enhanced with the number of regenerators. Therefore, to evaluate the system performance improvement, it is necessary toconsider the cumulative effect of nonlinear signal and noise transformation along the line. To quantify the overall effect, we studied the capacity of the source-destination transmission as a function of SNR that incorporates the resulting power of all added noise at the source-destination link. We stress that the proposed channel model is fundamentally different from decode-and-forward (since regenerators are memory-less) and does not assume any decoder/encoder pair to be used with in-line elements. Note that regenerators reduce noise and do not amplify the signal. An additional noise induced from a device itself $N_{\mathrm{R}}$ can be incorporated by substituting: $N \rightarrow N+R N_{\mathrm{R}}$.

In the discrete-time case, we simulated $2^{25}$ equi-probable symbols. One can see that for small switching windows discrete-time-effective noise source approximation allows capturing the behavior of the system (see 4.5). Increasing the switching time window leads to SER degradation due to accumulation of timing jitter caused by nonlinear transformation. The investigation of a timing jitter will be studied elsewhere. Here, we focus on characterizing the maximum transmission rate that can be achieved by regeneration with suppression of both time jitter and noise distortions; therefore, we are interested in optimizing the regenerative element to achieve the highest performance. Under this condition, a discrete-time approximation enables correct description of the system. Further, throughout the thesis, we have focused on this approach.

Further, we developed the general mathematical method of constructing and optimizing the set of transfer functions $y^{\prime}=T(y)$ (see, for example, Fig. 4.7) that can increase nonlinear transmission system capacity beyond the capacity of the linear AWGN channel. 

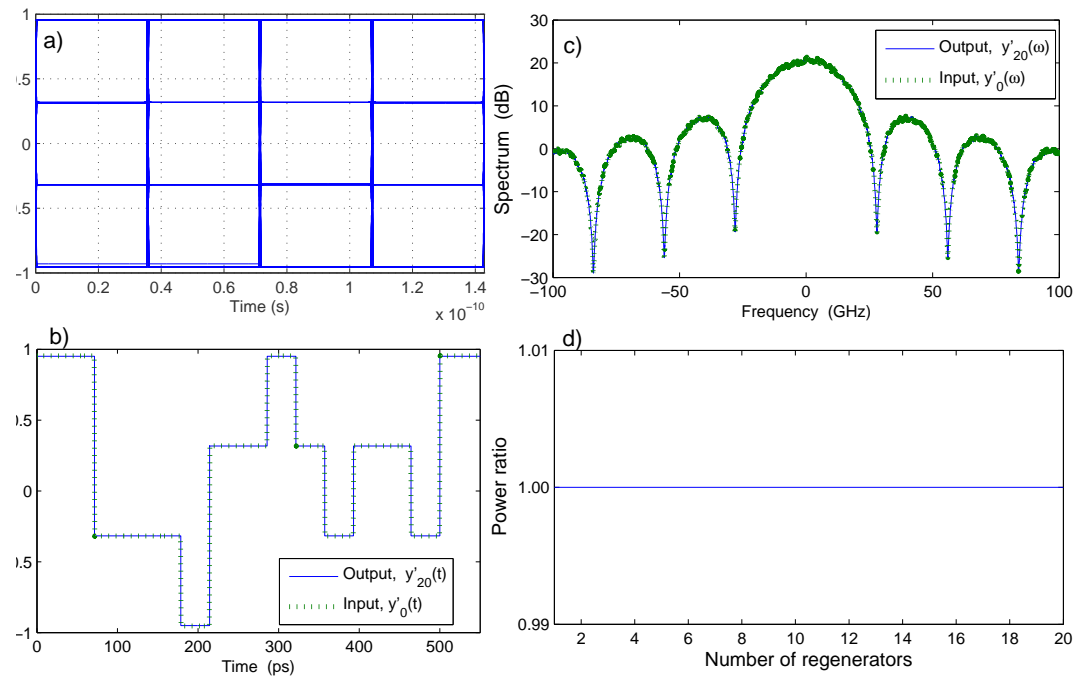

Figure 4.2: Waveforms for noiseless transmission via $R=20$ regenerators - a) Eyediagram, b) waveform, and c) spectrum of the signal at the output of the last regenerator (blue solid line) plotted alongside the input signal (green dashed line); d) power ratio of the output signal power at the last regenerator to the power of input signal
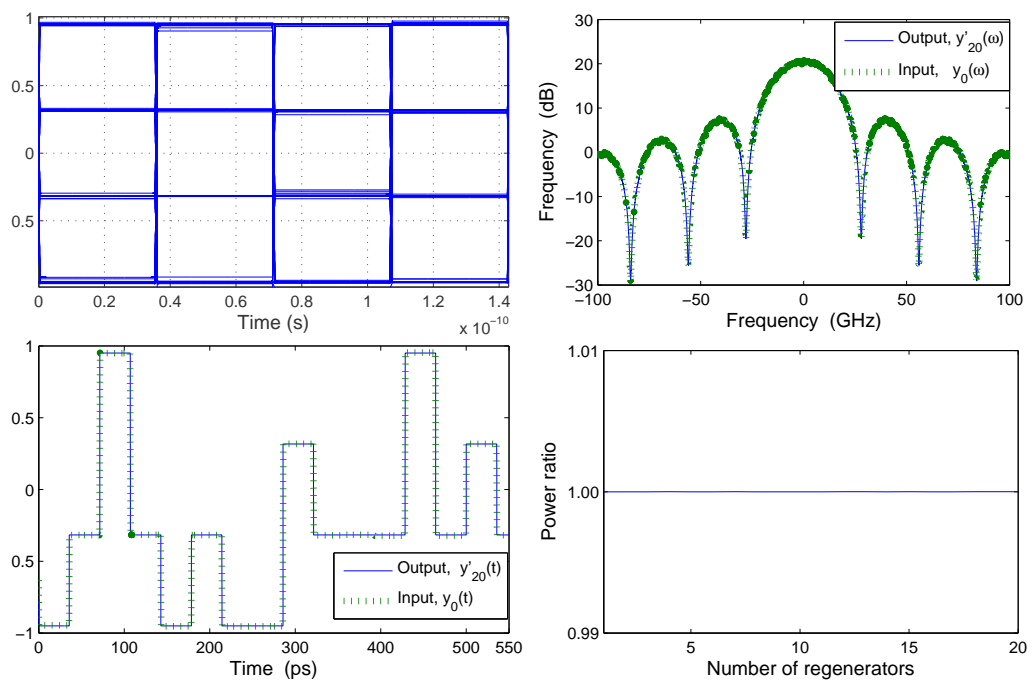

Figure 4.3: Waveforms for noisy $(O S N R=10 \mathrm{~dB})$ transmission via $R=20$ regenerators - a) Eye-diagram, b) waveform, and c) spectrum of the signal at the output of the last regenerator (blue solid line) plotted alongside the input signal (green dashed line); d) power ratio of the output signal power at the last regenerator to the power of input signal 

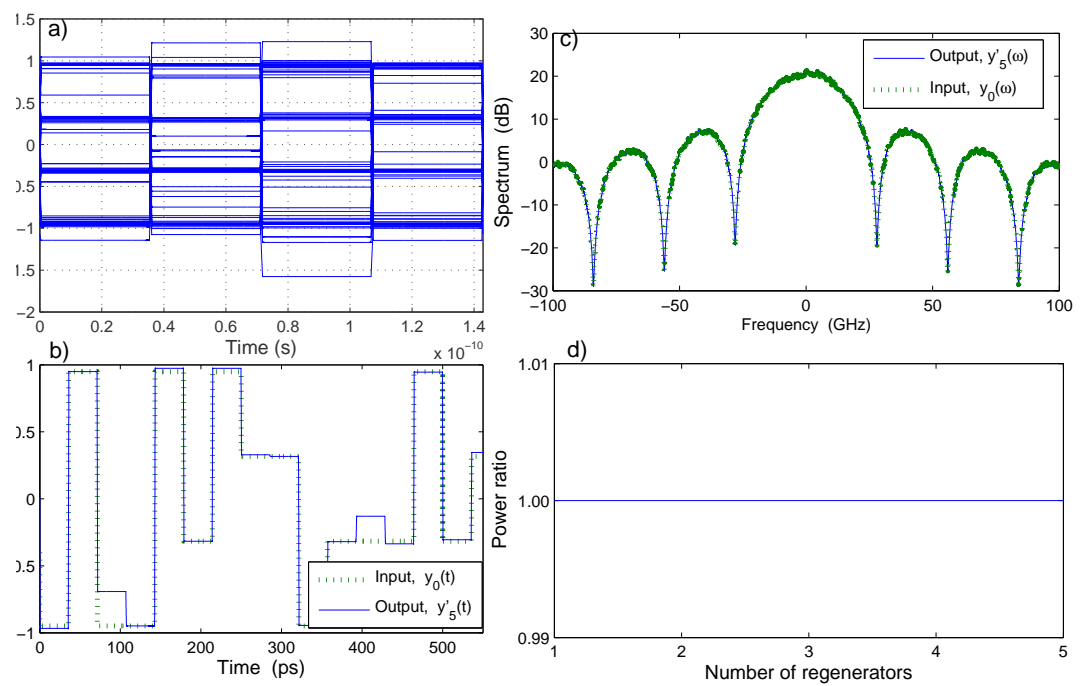

Figure 4.4: Waveforms for noisy $(O S N R=10 \mathrm{~dB})$ transmission via $R=5$ regenerators - a) Eye-diagram, b) waveform, and c) spectrum of the signal at the output of the last regenerator (blue solid line) plotted alongside the input signal (green dashed line); d) power ratio of the output signal power at the last regenerator to the power of input signal

\subsection{Regenerative mapping}

Here we propose a general model - regenerative mapping (see Fig. 4.6a,b) for designing the classes of nonlinear communication channels with capacity exceeding Shannon capacity of the linear AWGN channel. An important new feature introduced by the considered nonlinear mapping is the possibility of signal regeneration without requiring ideal regenerators (72) characterized by step-like piecewise transfer functions. Regenerative mapping incorporates experimental regenerative models and enables analytical optimization. Whenever the nonlinear transformation has multiple fixed points (Fig. 4.6c), the consequent interleaving of the accumulating noise with the nonlinear regenerator will produce effective noise suppression (see Fig. 4.6d, Fig. 4.8). In other words, the nonlinear regenerator will attract a signal to the closest fixed points and suppress the effective diffusion. The created washboard potential (Fig. 4.6c) quantizes the signal and improves transmission with consequent capacity increase. Similar ideas have been discussed in multiple contexts from physical systems (for superconductivity (153)-(154)) to the interpretation of biological memory effects in terms of potentials 


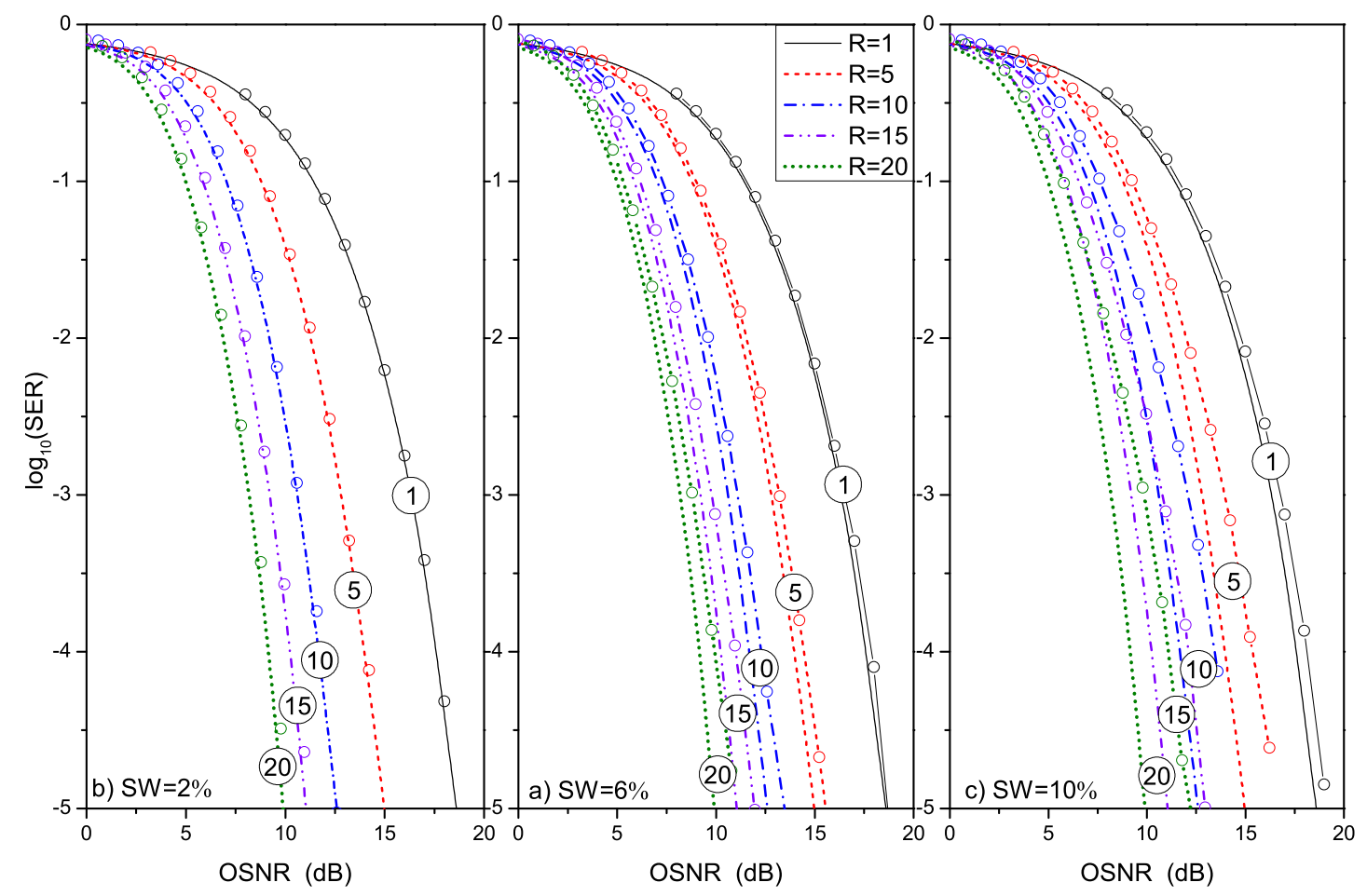

Figure 4.5: Comparison of discrete-time and continuous-time modelling - SER as a function of OSNR for different values of $S W$ - percentage of the switching window duration with respect to the symbol period. Discrete-time simulations (solid lines) show the upper limit of regenerative continuous-time transmission (shown by lines with circles), which can be achieved with small switching windows. The increase of $S W$ leads to performance degradation. The number of regenerative elements $R$ (with nonlinear TF: $\left.T(y)=y+\pi^{-1} \sin (\pi y)\right)$ was varied. 
with multiple minima (155).

\subsubsection{Regenerative functions}

We distinguish between ideal regenerator characterized by step-wise TF and regenerator with smooth TF.

In case of the ideal regenerator, if signal distortion $\delta$ is smaller than the corresponding regenerative area (width of the $k$-th step of the step-wise TF): $\delta<\left\|S_{k}\right\|$, then distortion is completely suppressed. Therefore, the width of the step should be chosen as the Voronoi region. Thus, the ideal regenerator, having the optimum width of regenerative area and flat plateau (removes noise completely if $\delta<\left\|S_{k}\right\|$ ), represents the highest efficiency regenerator. Ideal regenerator can be achieved opto-electronically or by placing regenerators with smooth TF in cascades: $y^{\prime}=T(T(T(. . T(y))) \ldots$ A number of transformations depends on the particular TF type, in particular for $T(y)=$ $y+1 / 3 \sin (3 y)$ one can approximate ideal step-wise TF closely by varying the number of applied transformations $N_{T}$ (see Fig. 4.9). Note, that one needs to place a nonlinear element in cascade, whereas only a single optical gate (after nonlinear signal transformations) is required (see Fig. 4.1).

Regenerators with smooth TF can be achieved by all-optical regenerative elements, which create a nonlinear response that needs to be optimized and designed in such a way that noise-induced distortions are reduced after transformations (see optimization technique further).

Regenerator reduces signal distortions which do not exceed the certain level - the size of the corresponding regenerative area: $|\delta|<|| S_{k}||$. So that under such conditions distortion after transformation $\delta^{\prime}$ is smaller than before: $\left|\delta^{\prime}\right|<|\delta|$ or $\left|\delta^{\prime} / \delta\right|<1$. If the nonlinear TF has a plateau level then within it the distortion is completely suppressed: $\delta^{\prime} / \delta=0$. However, plateau, though being an optimal case is not necessary, because for effective noise squeezing, the condition $\left|\delta^{\prime} / \delta\right|<1$ is sufficient. Consequently, to achieve noise reduction in the vicinity of an alphabet point $x$ one needs: $\left|T^{\prime}(x)\right|<1$ to be fulfilled - this is a suboptimal or stable case. If $T^{\prime}(x)=0$ then there is a plateau around the alphabet point - this is the best case and it is further referred as optimal or superstable. 


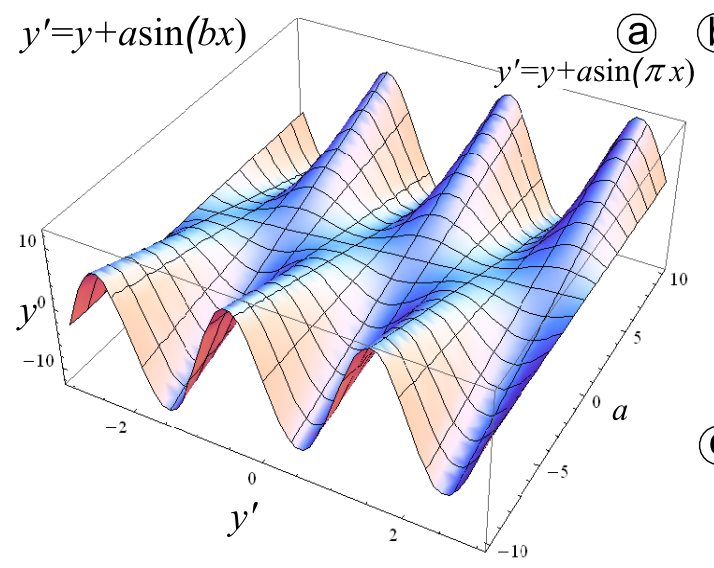

(b)

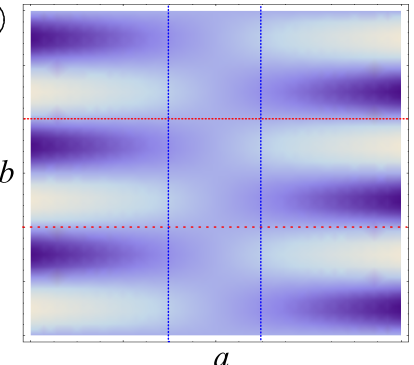

(C)
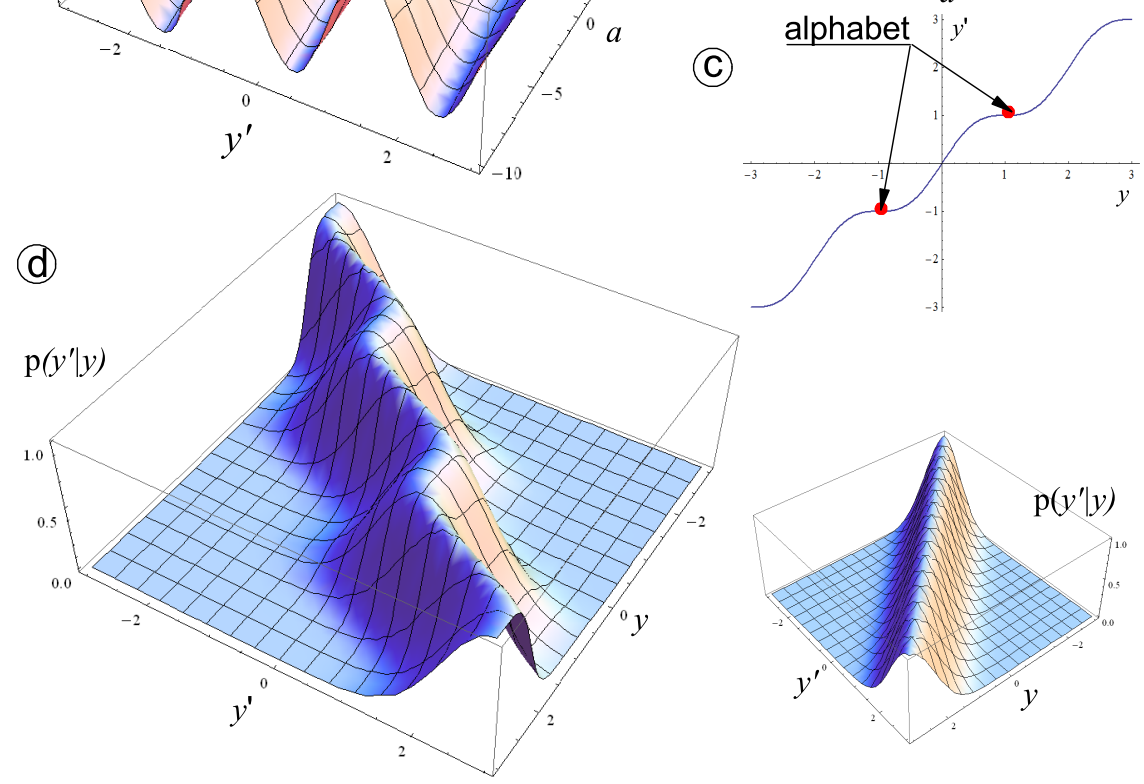

Figure 4.6: The regenerative sine transfer function (TF) - $y^{\prime}=T(x)=y+a \sin (b y)$. (a) plotted with varying parameter $a$ and fixed $b=\pi$ shows the periodic washboard potential, where the slope around fixed points (alphabet) is defined by the parameters of the transformation; (b) TF as a function of the parameters $a$ and $b$ given $y=\pi$ demonstrates the periodic dependence on $b$ and intensity defined by $a$ (higher intensity is shown by lighter color, whereas optimal parameter values are shown by dashed lines), (c) $y^{\prime}=T(y)$ for the parameters $b=\pi$ and $a=1 / b$ - the optimal set of parameters defines the zero-slope at the stationary point and maximum ability of noise suppression; (d) Gaussian pdf $p\left(y^{\prime} \mid y\right)$ associated with the linear channel (right panel) and the regenerative channel (left panel) having one sine-regenerator with the TF $y^{\prime}=y+\pi^{-1} \sin (\pi y)$ illustrates the effect of the $\mathrm{TF}$ on the stochastic properties of the channel. 


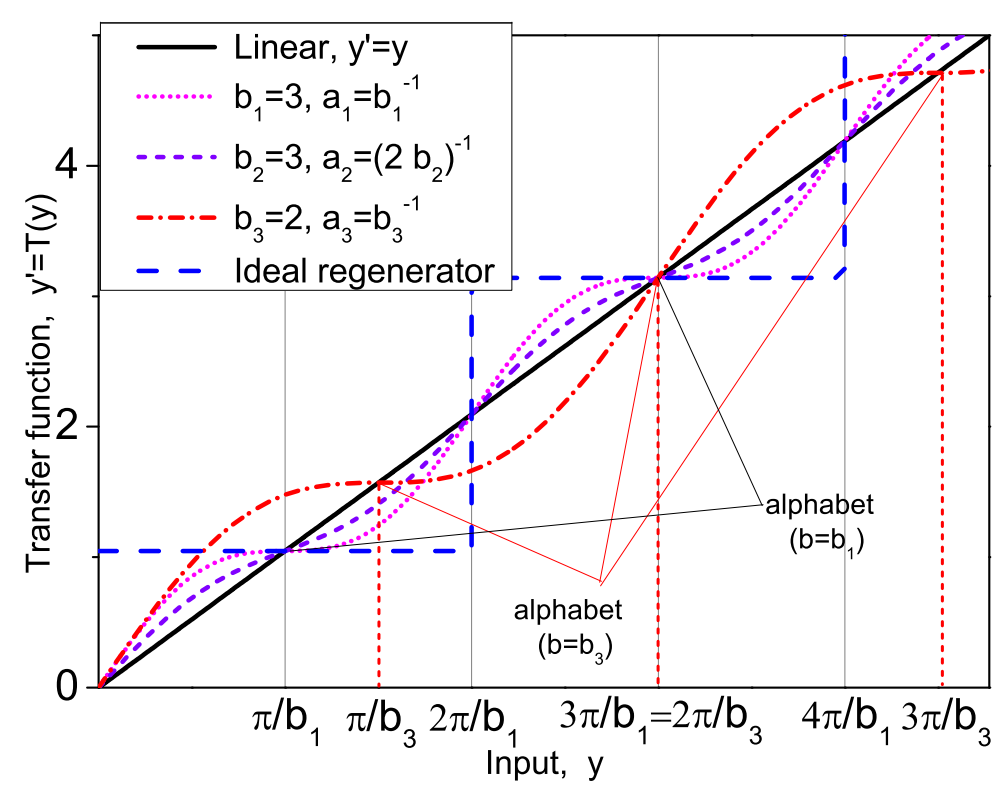

Figure 4.7: Regenerative transfer functions - plotted for the ideal regenerator and sinemapping: $y^{\prime}=y+a \sin (b y)$

Of course, a regenerative transformation should not affect alphabet points, that is, $T(x)=x$, so one can place a number of regenerators in-line in cascade. Finally, it is optimum to place an alphabet point in the center of the corresponding regenerative region, theorem, where an alphabet point is an inflection point of the transformation: $T^{\prime \prime}(x)=0$.

The regenerative transformations result in the effective periodic potential which creates attraction regions in the signal mapping without making the hard decision. Where the points are "attracted" to the alphabet, it should remain stable. This results in the following set of conditions imposed on the transfer function:

$$
T[x]=x, \quad T^{\prime \prime}[x]=0, \quad\left|T^{\prime}[x]\right|<1
$$

The first expression implies that the alphabet is defined by the stationary points $x^{*}$ of the mapping. Next, the transfer function should change curvature at the alphabet, that is, the alphabet point is the center of the attraction region. The third expression reflects stability, so that the signal point distortion is effectively suppressed. When the first derivative is equal to zero, the alphabet is superstable. 

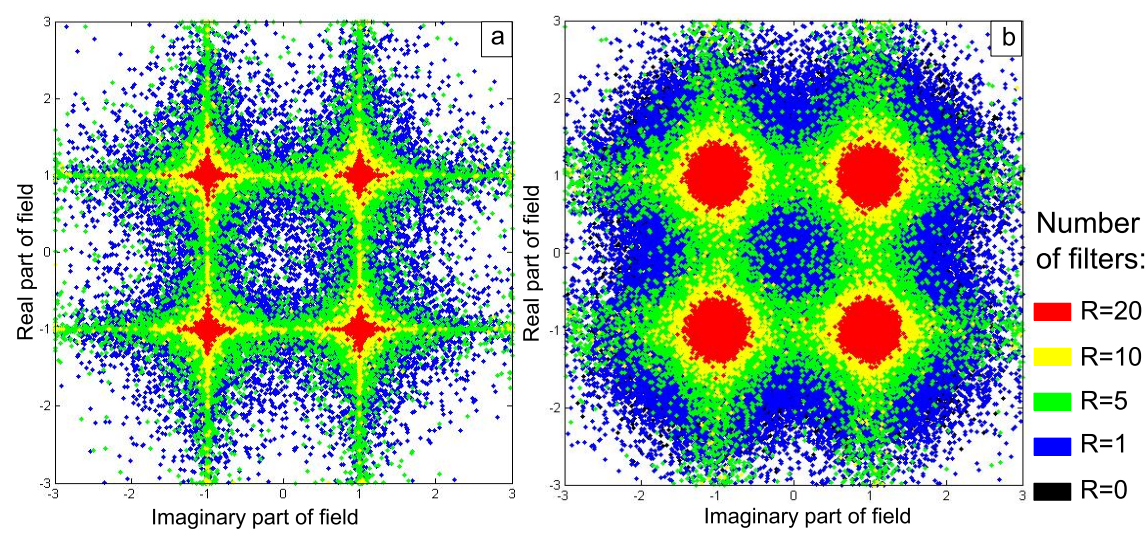

Figure 4.8: Transmission improvement - Square $K=4$ constellation a) at the output of the $R$-th regenerator $Y_{R}^{\prime}=T\left(Y_{R}\right)$ and b) at the receiving end $Y_{R+1}=T\left(Y_{R}\right)+\eta_{R+1}$ (see Fig. 4.1 for the transmission lines having AWGN distortion of $S N R=3 \mathrm{~dB}$ with the different number of sine-regenerators characterized by TF: $y^{\prime}=T(y)=y+\pi^{-1} \sin (\pi y)$. Linear case $R=0$ is shown for reference. One can see that, though the distortion is caused by AWGN with normal Gaussian distribution, the statistical properties after transformation are changed (compare signal at the output of the regenerator [panel a] when noise statistics are no longer Gaussian with signal when AWGN is added again [panel b]). This demonstrates that the higher capacity is due to the induced changes in noise distribution, which are caused by nonlinear signal transformation (without signal amplification). Note, transformation is applied independently to both signal quadratures and the problem can viewed as transformation induced upon two one-dimensional lattices independently. 


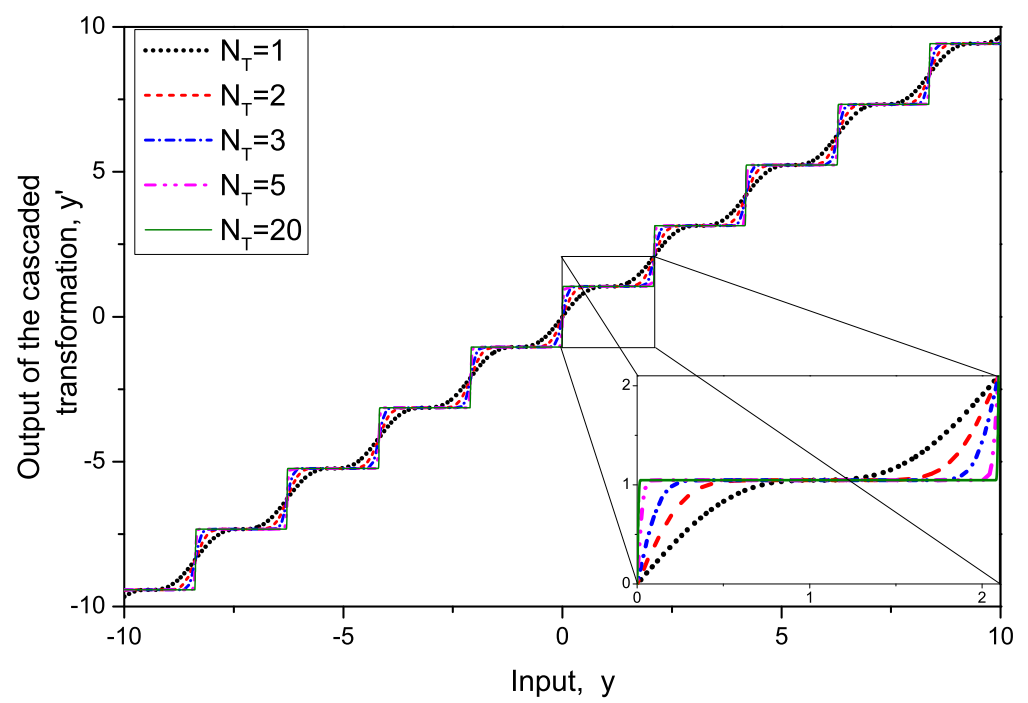

Figure 4.9: The regenerative functions - approximation of ideal regenerator - using smooth transfer functions (here $T(y)=y+1 / 3 \sin (3 y)$ ) and applying them in consequence $N_{T}$ times: $y^{\prime}=T(T(T(. . T(y)))$.. one can closely approach an ideal step-wise TF.

\subsubsection{Regenerative Fourier transform model}

To demonstrate the constructive role of nonlinearity without necessarily "hard decision", we propose the nonlinear regeneratoring element that transforms the input $x$ into the output $y$ thusly: $y^{\prime}=T(y)=y+a \sin (b y)$. The model is commonly referred to in theoretical physics as a sine-mapping $(156,157)$. The transfer function represents the first order expansion in the Fourier series of the step-wise ideal regenerative TF; thus, it is a close, smooth approximation of the ideal regenerator - regenerative Fourier transform (RFT). The step-wise TF of an ideal regenerator with step $x_{0}$ is given by: $\left[y / x_{0}\right]$ where [ ] represents the integer part of the variable. One can represent it as

$$
y_{\text {ideal }}^{\prime}=\left[\frac{y}{x_{0}}\right]=y-\bmod \left(y, 2 x_{0}\right)+x_{0}
$$

or expanding mod operator in the Fourier series expansion one gets:

$$
y_{\text {ideal }}^{\prime}=\left[\frac{y}{x_{0}}\right]=y-\left(x_{0}-\frac{2 x_{0}}{\pi} \sum_{k=1}^{\infty} \frac{\sin \left(\frac{\pi k y}{x_{0}}\right)}{k}\right)+x_{0}
$$

after leaving only first-order term in the series:

$$
y_{\text {sine }}^{\prime}=T(y)=y+\frac{2 x_{0}}{\pi} \sin \left(\frac{\pi y}{x_{0}}\right)
$$


and notating $\pi / x_{0}$ as $b$ one obtains:

$$
y_{\text {sine }}^{\prime}=T(y)=y+a \sin (b y)
$$

where alphabet is given by $x_{k}=(2 k+1) x_{0}=(2 k+1) \pi / b$ with $k \in \mathrm{Z}$.

Note, the transformation is applied to each signal quadrature independently (here $y$ is a real variable, which describes both real or imaginary parts of a complex signal):

$$
y^{\prime}=T(y)=y+a \sin (b y)
$$

The mapping RFT is shown in Fig. 4.7. According to the previously described optimization procedure, in the considered example, the alphabet is placed at the points $\pi(2 k+1) / b$ where $k \in \mathrm{Z}$ that are stable if and only if $0<a b<2$ (in particular, if $a=0$, the channel transforms to a linear AWGN channel). The last expression asserts that noise after the transformation is reduced. Note that, in particular, the system is superstable when $a b=1$; this gives the optimum parameter values, whereas inequality defines the suboptimal parameter range. The proposed model enables regeneration of multilevel modulation formats.

\subsection{Upper bound of regeneration efficiency - capacity of the channel with ideal regenerators}

Shannon capacity of the considered systems is a function of SNR, the number of regenerators $R$ and the parameters of nonlinear mapping. We start the capacity analysis with the case of ideal regenerators (characterized by step-wise transfer functions) and then proceed to regenerators with smooth transfer function, which allow approximating the ideal case by implementing all-optical regeneration (145)-(148).

Ideal regenerators assign each transmitted symbol to the closest element of the given alphabet, so that the diffused point $x^{\prime}$ (originated from the input point $x_{l}$ ) will be assigned to the closest neighbor $x_{k}$ in the regenerative area $S_{k}$. The ideal regenerator has the largest area $S_{k}$ - Voronoi region given by $\left(x_{k-1}+\frac{x_{k}-x_{k-1}}{2}, x_{k}+\frac{x_{k+1}-x_{k}}{2}\right)$. Capacity analysis of the system with the ideal regenerators defines the upper bound of 
regeneration efficiency. The conditional probability of such a system is defined through the matrix elements (72):

$$
P\left(y=x_{k} \mid x_{l}\right)=\int_{S_{k}} d x^{\prime} p_{G}\left(x^{\prime} \mid x_{l}\right)=W_{k l}, \quad p_{G}\left(x^{\prime} \mid x_{l}\right)=\frac{1}{\sqrt{2 \pi N / R}} \exp \left(-\frac{\left(x^{\prime}-x_{l}\right)^{2}}{2 N / R}\right)
$$

The transition matrix is defined as follows

$$
W_{k l}=\int_{x_{k-1}+\frac{x_{k}-x_{k-1}}{2}}^{x_{k}+\frac{x_{k+1}-x_{k}}{2 \pi N / R}} d x^{\prime} \frac{1}{\sqrt{2 \pi N / R}} \exp \left(-\frac{\left(x^{\prime}-x_{l}\right)^{2}}{2 N / R}\right)=\frac{1}{2}\left(\operatorname{erf}\left[\Delta_{k l}^{+}\right]-\operatorname{erf}\left[\Delta_{k l}^{-}\right]\right)
$$

with

$$
\Delta_{k l}^{ \pm}=\left(x_{k}+x_{k \pm 1}-2 x_{l}\right) \sqrt{\frac{R}{8 N}},
$$

We assume in this section that the last $R$-th regenerator is placed at the receiver (Fig. 4.10); therefore, compared to the scheme in Fig. 4.1, there is not $R+1$, but $R$ effective noise sources. An effective noise source $\eta_{m}$ represents the noise added to the signal during propagation via the $m$-th span (it can be considered as noise resulting from multiple EDFAs or from Raman amplification). Since in both cases the noise power $N$ added to the signal during transmission is the same, therefore, the noise power at each node is $N_{m}=N / R$ (whereas in the case plotted in Fig. 4.1, further used for modelling regenerators with smooth nonlinear TF, there is noise added after $R$-th regenerator; therefore, at each node, the noise power is $\left.N_{m}=N / R\right)$. Note, both channels have the same power of input signal $S$ and power of noise added during transmission $N$, as well as the same number of regenerators $R$; however, the positioning of the regenerators and models are different.

Note, that the essential difference between linear and regenerative channels is that the latter is fundamentally discrete. An ideal regenerator attracts a signal point $x^{\prime}$ to the closest alphabet point $x_{k}$, so it means that an effective attractive region is created $S_{k}$. In capacity calculations, the alphabet is subject to optimization, whereas the choice of alphabet defines $S_{k}$. In adapted here consideration of one-dimensional lattices, $S_{k}$ is a region given by a starting point $x_{k-1}+\frac{x_{k}-x_{k-1}}{2}$ and ending point $x_{k}+\frac{x_{k+1}-x_{k}}{2}$.

The size of the attractive region $S_{k}$ has a direct impact on the regenerative properties; that is, if the noise-induced distortion is large, so that the distorted point (originated from an alphabet point $\left.x_{k}\right)$ is shifted out of its original attraction region $\left(S_{k}\right)$, then it will be attracted to the wrong alphabet point; consequently, this eventually will cause 

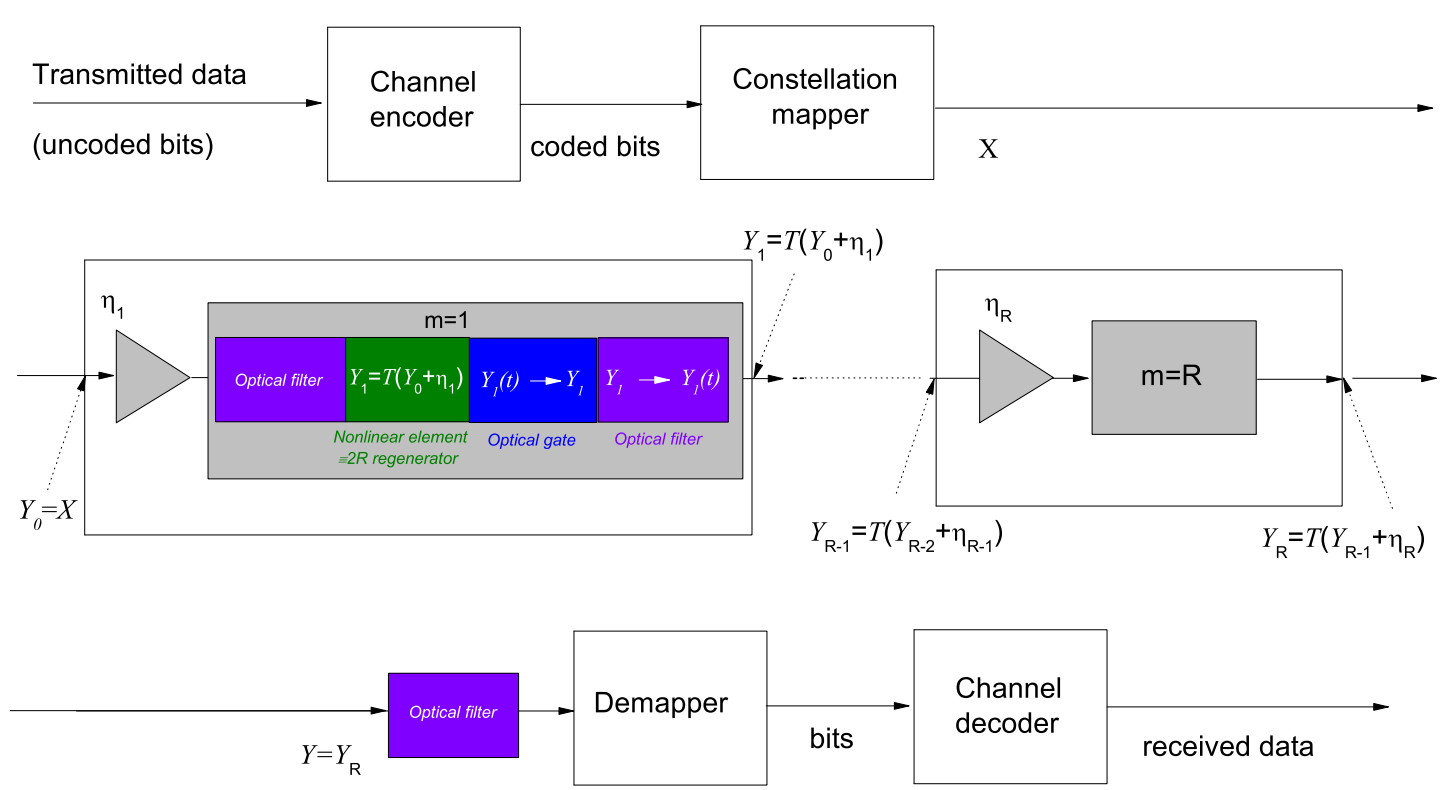

Figure 4.10: The regenerative channel model -

an error at the receiver. In other words, if $S_{k}$ is too small, then the regenerator will only increase distortion and regeneration will have a negative impact. In particular, if $S_{k}$ is put to zero, the regenerative effect vanishes. As $S_{k}$ has a meaning of the cell size (distance between the neighboring alphabet points) in the case of an ideal regenerator (which ensures maximum regeneration efficiency), the optimal amplitude distribution is discrete. Thus, the size of $S_{k}$ depends on the noise variance added at each span. Noise with the same variance is added to all transmitted points, independently on their values; therefore, the size of $S_{k}$ is independent of the value of $x_{k}$. Consequently, the optimum alphabet consists of equidistant points with spacing $d=x_{k+1}-x_{k}$ for any $k$ and the value of $d$ depends only on the variance of the noise added at each span. This reasoning was supported by numerical simulations, when a value of $d$ was subject to optimization for given values of signal $S$, total accumulated noise power $N$, and the number of regenerators in-line $R$. It was shown that the optimum value of $d$ is a function $N$ and $R$ (see next section for more details).

As we place a number of identical regenerators in cascade along the line, due to the Markovian nature of the stochastic system, the overall transition matrix after $R$ regenerative segments reads as $\mathbf{M}=\mathbf{W}_{1}\left(N_{1}\right) \ldots \mathbf{W}_{m}\left(N_{m}\right) \ldots \mathbf{W}_{R}\left(N_{R}\right)$ for a fixed total noise 
variance $N=\sum_{m=1}^{R} N_{m}$. From a symmetry (that is, replacing regenerators will not affect the product and since $\mathbf{W}_{m}\left(N_{m}\right)$ is a monotonically decreasing function of $N_{m}$, it is reasonable to consider regenerators to be placed equidistantly along the line with the same noise variance added at each span: $N_{m}=N / R=$ const (since in this case the noise variance at each span is minimized).

Under these assumptions, the overall transition matrix after $R$ regenerative segments is given by

$$
\mathbf{M}=\mathbf{W}^{R}
$$

Using conditional pdf, one can calculate the Shannon capacity of the channel.

\subsubsection{Analytical calculations of the capacity of the regenerative channel}

Applying the definition of Eq. 4.7 to the binary case (that is, points are given as $x_{+}=-x_{-}=\sqrt{S}$, where $S$ notates the signal power), we obtain the transition matrix of one span for binary channel:

$$
\underline{\mathbf{W}}=\left(\begin{array}{ll}
w_{+} & w_{ \pm} \\
w_{ \pm} & w_{-}
\end{array}\right)
$$

with matrix elements given by:

$$
\begin{gathered}
w_{+}=\int_{S_{+}} d x^{\prime} p_{G}\left(x^{\prime} \mid \sqrt{S}\right)=\int_{0}^{\infty} d x^{\prime} \frac{1}{\sqrt{2 \pi N / R}} \exp \left(-\frac{\left(x^{\prime}-\sqrt{S}\right)^{2}}{2 N / R}\right)=\frac{1}{2}(1+\operatorname{erf}[\sqrt{R \cdot S N R / 2}]) \\
w_{ \pm}=\int_{S_{ \pm}} d x^{\prime} p_{G}\left(x^{\prime} \mid \sqrt{S}\right)=\int_{-\infty}^{0} d x^{\prime} \frac{1}{\sqrt{2 \pi N / R}} \exp \left(-\frac{\left(x^{\prime}-\sqrt{S}\right)^{2}}{2 N / R}\right)
\end{gathered}
$$

Similarly, for the second point $x_{-}=-\sqrt{S}$. Obviously, from a symmetry of the channel: $w_{+}=w_{-}=1-w_{ \pm}$. After transmission via $R$ regenerative segments the overall transition matrix reads as $\underline{\mathbf{M}}=\underline{\mathbf{W}}^{R}$ :

$$
\underline{\mathbf{M}}=\left(\begin{array}{ll}
m_{+} & m_{ \pm} \\
m_{ \pm} & m_{-}
\end{array}\right)
$$

with matrix elements: diagonal $m_{+}=m_{-}=([1+\operatorname{erf}[\sqrt{R \cdot S N R / 2}]] / 2)^{R}$ and nondiagonal $m_{ \pm}=1-m_{+}$. 
At low SNR range, the channel is binary in each of $n$ dimensions. Using the definition of capacity per dimension for the discrete-input, discrete-output channels:

$$
\underline{C_{R}}=H_{y}-H_{y \mid x}=-\sum_{k=1}^{K} Q_{k} \log _{2} Q_{k}+\sum_{k=1}^{K} P_{k} \sum_{l=1}^{K} W_{k l} \log _{2} W_{k l}
$$

Therefore, capacity per dimension is well approximated by the following expression:

$$
\begin{gathered}
\underline{C_{R}}=-\left(\frac{1}{2} \log _{2} \frac{1}{2}+\frac{1}{2} \log _{2} \frac{1}{2}\right)+\frac{2}{2}\left(m_{+} \log _{2} m_{+}+m_{ \pm} \log _{2} m_{ \pm}\right) \\
=\left(1+m_{+} \log _{2}\left(m_{+}\right)+m_{ \pm} \log _{2}\left(m_{ \pm}\right)\right)
\end{gathered}
$$

with the transition matrix elements: diagonal $m_{+}=m_{-}$and non-diagonal $m_{ \pm}=1-$ $m_{+}$.

As SNR rises, the closest neighbors distance $\left(d=x_{k+1}-x_{k}\right)$ reaches the optimal cell size $d=d_{\text {opt }}$, which defines the optimum size of the decision boundary determined by the noise variance and the number of in-line regenerators. So, as SNR rises, alphabet points are distributed equidistantly with the constant closest neighbor distance $d_{\text {opt }}$. Thus, at high SNR, the system is characterized by the optimal decision boundaries that are sufficiently large compared with the variance for effective noise suppression. Therefore, with the growing signal power, the amplitude distribution of $K$-points alphabet $x_{k=1 . . K}$ remains constant (that is, equidistant with the closest neighbors distance $d_{o p t}$ ), whereas the maximum entropy principle defines Gaussian distribution as the optimal probability for a fixed average energy constraint. Thereafter, the output probability can be well approximated as $Q_{k}=\mu e^{-v x_{k}^{2}}$, here constants are chosen to satisfy conditions $\sum_{k=1}^{K} Q_{k}=1$ and $\sum_{k=1}^{K} Q_{k} x_{k}^{2}=S+N / R$. In the limit of high SNR and/or large number of regenerators, so that the dimension-less parameter $\Delta=d \sqrt{R / 8 N} \gg 1$, the noise is sufficiently squeezed and the faulty decision occurs only between the nearest neighbors. Further, we consider the problem in the approximation of the closest neighbors, so the transition matrix of the span has the form:

$$
\overline{\mathbf{W}}=\left(\begin{array}{ccccccccc}
\ldots & \ldots & \ldots & \ldots & \ldots & \ldots & \ldots & \ldots & \ldots \\
\ldots & 0 & \bar{w} & \widetilde{w} & \bar{w} & 0 & \ldots & \ldots & \ldots \\
\ldots & \ldots & 0 & \bar{w} & \widetilde{w} & \bar{w} & 0 & \ldots & \ldots \\
\ldots & \ldots & \ldots & 0 & \bar{w} & \widetilde{w} & \bar{w} & 0 & \ldots \\
\ldots & \ldots & \ldots & \ldots & \ldots & \ldots & \ldots & \ldots & \ldots
\end{array}\right)
$$


with the elements given by

$$
\widetilde{w}=\int_{S_{+}} d x^{\prime} p_{G}\left(x^{\prime} \mid \sqrt{S}\right)=\int_{x_{k}-d / 2}^{x_{k}+d / 2} d x^{\prime} \frac{1}{\sqrt{2 \pi N / R}} \exp \left(-\frac{\left(x^{\prime}-x_{k}\right)^{2}}{2 N / R}\right)=\operatorname{erf}(\Delta)
$$

and $\bar{w}=(1-\widetilde{w}) / 2$. In this approximation, the overall transition matrix $\overline{\mathbf{M}}$

$$
\overline{\mathbf{M}}=\left(\begin{array}{ccccccccc}
\ldots & \ldots & \ldots & \ldots & \ldots & \ldots & \ldots & \ldots & \ldots \\
\ldots & 0 & \bar{m} & \widetilde{m} & \bar{m} & 0 & \ldots & \ldots & \ldots \\
\ldots & \ldots & 0 & \bar{m} & \widetilde{m} & \bar{m} & 0 & \ldots & \ldots \\
\ldots & \ldots & \ldots & 0 & \bar{m} & \widetilde{m} & \bar{m} & 0 & \ldots \\
\ldots & \ldots & \ldots & \ldots & \ldots & \ldots & \ldots & \ldots & \ldots
\end{array}\right)
$$

with $\widetilde{m} \simeq \widetilde{w}^{R} \simeq \lim _{\Delta \rightarrow \infty} \widetilde{w}^{R} \simeq 1-R e^{-\Delta^{2}} / \Delta / \sqrt{\pi}$ and $\bar{m}=(1-\widetilde{m}) / 2$. The conditional entropy is given by definition:

$$
H_{y \mid x}=-\sum_{k=1}^{K} P_{k} \sum_{l=1}^{K} M_{l k} \log _{2} M_{l k}
$$

Under the assumption of the closest neighbors, the formula is simplified to:

$$
\lim _{S N R \cdot R \rightarrow \infty} H_{y \mid x}=-\sum_{k=1}^{K} P_{k}\left(\widetilde{m} \log _{2}(\widetilde{m})+2 \bar{m} \log _{2}(\bar{m})\right) \simeq-\left(\widetilde{m} \log _{2}(\widetilde{m})+2 \bar{m} \log _{2}(\bar{m})\right)
$$

In the operating limit $\Delta \gg 1$, consequently, $\operatorname{Re}^{-\Delta^{2}} / \Delta / \sqrt{\pi}$ is a small parameter. In this approximation, the conditional entropy equates to:

$$
\lim _{S N R \cdot R \rightarrow \infty} H_{y \mid x}=-R \frac{e^{-\Delta^{2}}}{\Delta \sqrt{\pi}} \log _{2}\left(R \frac{e^{-\Delta^{2}}}{4 \Delta \sqrt{\pi}}\right)
$$

By definition, the output entropy is given by:

$$
H_{y}=-\sum_{k=1}^{K} Q_{k} \log _{2}\left(Q_{k}\right)
$$

Based on the results of numerical simulations (see next subsection), we assume the output probabilities to be Gaussian: $Q_{k}=\mu e^{-v x_{k}^{2}}$, here, constants are chosen to satisfy conditions $\sum_{k=1}^{K} Q_{k}=1$ and $\sum_{k=1}^{K} Q_{k} x_{k}^{2}=S+N / R$. These reflect on the output entropy:

$$
\lim _{S N R \cdot R \rightarrow \infty} H_{y}=-\sum_{k=1}^{K} Q_{k} \log _{2}\left(Q_{k}\right)=-\sum_{k=1}^{K} Q_{k}\left(\log _{2}(\mu)-v x_{k}^{2}\right)
$$


using the conditions $\sum_{k=1}^{K} Q_{k}=1$ and $\sum_{k=1}^{K} Q_{k} x_{k}^{2}=S+N / R$ :

$$
\lim _{S N R \cdot R \rightarrow \infty} H_{y}=-\log _{2}(\mu)+v(S+N / R) \log _{2}(e)=\log _{2}\left(\mu e^{-v(S+N / R)}\right)
$$

One can find constants $\mu$ and $v$ using quasi-continuous approximation (that is, in the continuum limit), assuming that there are large numbers of points situated such that the maximal distance between them is much larger than the minimal distance. Then the sums can be considered as integrals with a proper weight - the step size, that is, distance between the adjacent points (similar to the Riemann integral):

$$
\sum_{k=1}^{K} Q_{k} \rightarrow \frac{1}{d} \int_{-\infty}^{\infty} q(y)=\frac{1}{d} \int_{-\infty}^{\infty} d y \mu e^{-v y^{2}}=\frac{\mu}{d} \sqrt{\frac{\pi}{v}}=1
$$

and

$$
\sum_{k=1}^{K} Q_{k} x_{k}^{2} \rightarrow \frac{1}{d} \int_{-\infty}^{\infty} d y q(y) y^{2}=\frac{1}{d} \int_{-\infty}^{\infty} \mu e^{-v y^{2}} y^{2}=\frac{\mu}{2 \mu d} \sqrt{\frac{\pi}{v}}=\sum_{k=1}^{K} Q_{k} x_{k}^{2}=S+N / R
$$

Thus, we derive that $\mu=d \sqrt{v / \pi}$ and $v=(2(S+N / R))^{-1}$. Substituting the obtained parameters in Eq. 4.26, we derive:

$$
\lim _{S N R \cdot R \rightarrow \infty} H_{y}=\frac{1}{2} \log _{2}\left(\frac{2 \pi e(S+N / R)}{d_{o p t}^{2}}\right)
$$

The capacity is given by maximizing the difference of the aforementioned entropies over the optimum cell size

$$
C=\max _{d_{o p t}}\left(H_{y}-H_{y \mid x}\right)_{S N R \cdot R \rightarrow \infty}=\frac{1}{2} \log _{2}\left(\frac{2 \pi e(S+N / R)}{d_{o p t}^{2}}\right)+R \frac{e^{-\Delta^{2}}}{\Delta \sqrt{\pi}} \log _{2}\left(R \frac{e^{-\Delta^{2}}}{4 \Delta \sqrt{\pi}}\right)
$$

The optimum cell size can be found as follows:

$$
\left(\frac{1}{2} \log _{2}\left(\frac{2 \pi e(S+N / R)}{d_{o p t}^{2}}\right)+R \frac{e^{-\Delta^{2}}}{\Delta \sqrt{\pi}} \log _{2}\left(R \frac{e^{-\Delta^{2}}}{4 \Delta \sqrt{\pi}}\right)\right)_{d_{o p t}}^{\prime}
$$

which can be solved numerically or approximated analytically as follows:

$$
\left(H_{y \mid x}\right)_{d_{o p t}}^{\prime}=0
$$


Solving

$$
\left(R \frac{e^{-\Delta^{2}}}{\Delta \sqrt{\pi}} \log _{2}\left(R \frac{e^{-\Delta^{2}}}{4 \Delta \sqrt{\pi}}\right)\right)_{d_{o p t}}^{\prime}=0
$$

we can approximate the solution of 4.32 by using an approximation function $\kappa$ :

$$
d_{o p t}^{2}=\frac{8 N \kappa}{R} \Omega\left(\frac{e^{2} R^{2}}{8 \pi \kappa}\right), \quad \kappa=1+10 R^{-1}
$$

here $\Omega$ is the so-called Omega function or Lambert function, also referred to as product logarithm. Note, that the considered channel is significantly discrete and the maximum closest neighbors distance depends on the noise properties and regeneration parameters.

Finally, the channel capacity in the limit $S N R \cdot R \rightarrow \infty$ is found as

$$
\lim _{S N R \cdot R \rightarrow \infty} C_{R}=\overline{C_{R}}=\frac{1}{2} \log _{2}\left(S N R+\frac{1}{R}\right)+\frac{1}{2} \log _{2}\left(\frac{2 \pi e N}{d_{o p t}^{2}}\right)+R \frac{e^{-\Delta^{2}}}{\Delta \sqrt{\pi}} \log _{2}\left(R \frac{e^{-\Delta^{2}}}{4 \Delta \sqrt{\pi}}\right)
$$

Thus, with the growing SNR, one can observe a constant gap that quantifies improvement between capacities of the regenerative and linear AWGN channels having the same power of input signal $S$ and the same power of AWGN added to the signal during transmission $N$. This is useful for benchmarking. The capacity improvement is defined by the noise variance and the number of regenerators:

$$
\lim _{S N R \cdot R \rightarrow \infty} \Delta C_{R}=\overline{C_{R}}-\frac{1}{2} \log _{2}(S N R+1)=\frac{1}{2} \log _{2}\left(\frac{2 \pi e N}{d_{o p t}^{2}}\right)+R \frac{e^{-\Delta^{2}}}{\Delta \sqrt{\pi}} \log _{2}\left(R \frac{e^{-\Delta^{2}}}{4 \Delta \sqrt{\pi}}\right)
$$

The minimum SNR value, when $d_{o p t}$ is achieved, defines the maximum capacity ratio to its linear analogue, that is, $S N R_{o p t}=d_{o p t}^{2} / 4 N$. At this $S N R$ value, both analytic formulae Eqs. 4.16 and 4.35 can be interpolated to describe capacity at the full range.

\subsubsection{Numerical simulations of the capacity of the regenerative chan- nel}

Using the conditional probability for ideal regenerators, one can optimize numerically mutual information as a function of input distribution: $\left\{x_{k}, P_{k}\right\}, k=1 . . K$, where $K$ is the constellation size. 


\subsection{Upper bound of regeneration efficiency - capacity of the channel with ideal}

regenerators

Since noise (AWGN) is added linearly to the signal, the resulting distortion will have the same stochastic properties for each signal point $k=1 . . K$. Thus, the regenerative region $S_{k}$ will be the same for all signal points. Consequently, we simulate points to be equidistant. Thus, theproblem is reduced to find the optimal set of $\left\{d, P_{k}, K\right\}$. Note, as we vary simultaneously $d$ and probability distribution $P_{k}$, this allows us to perform optimization thoroughly, since for small values of $d$ one can approximate continuous distribution. Also, if optimization returns $P_{k}=0$ for particular number $k$, it will result in non-equidistant distribution (since the distance between non-zero probability signal points will be non-equidistant): $x_{k+1}-x_{k-1} \neq d$. Thus, an approach to vary $d, P_{k}, K$ enables optimizing the problem thoroughly for any general case and, therefore, calculating the capacity as the maximum of mutual information functional for a given conditional probability Eq. 4.10.

The program for numerical simulations was written in Matlab using a built-in sequential quadratic programming (SQP) algorithm (written as part of fmincon Matlab function). SQP is based on the works of Biggs, Han, and Powell ((158) and references therein) and allows for constrained optimization using Newton's method. The parameters of the function were set to standard and termination tolerance on the function value was set to $10^{-6}$ (other parameters are: the relative first-order optimality measure is less than $10^{-6}$ and the relative maximum constraint violation is less than $10^{-6}$ ). The initial search point was chosen to be equi-probable probability distribution (it was verified that the change of initial values does not affect optimization result), the constraints were $\sum P_{k}\left|x_{k}\right|^{2} \leq S$ and $\sum P_{k}=1$. The input parameters were: $S N R$ and $R$. The number of signal points (constellation size $K$ ) was varied.

The numerical simulations showed that at low SNR the optimum constellation is binary with the distance between the alphabet points: $d=\sqrt{4 S}=\sqrt{4 S N R \cdot N}$. As SNR increases, the value of $d$ grows until $d_{\text {opt }}$ (see Fig. 4.11); a further increase of SNR beyond $S N R_{o p t}=d_{o p t}^{2} / 4 N$ leads to the expansion of the constellation, so that more points have non-zero probabilities; however, the distance between the points $d$ does not change. The explanation of the effect of the constant amplitude distribution at high SNR was given in the previous section. Any deviation from $d_{o p t}\left(d>d_{o p t}\right.$ or $\left.d<d_{o p t}\right)$ leads to MI degradation. In particular, if $d \rightarrow 0$, the regeneration effect is negative. The comparison of thenumerical simulation results with analytical formula Eq. 4.34 is shown in the inset if Fig. 4.11. 


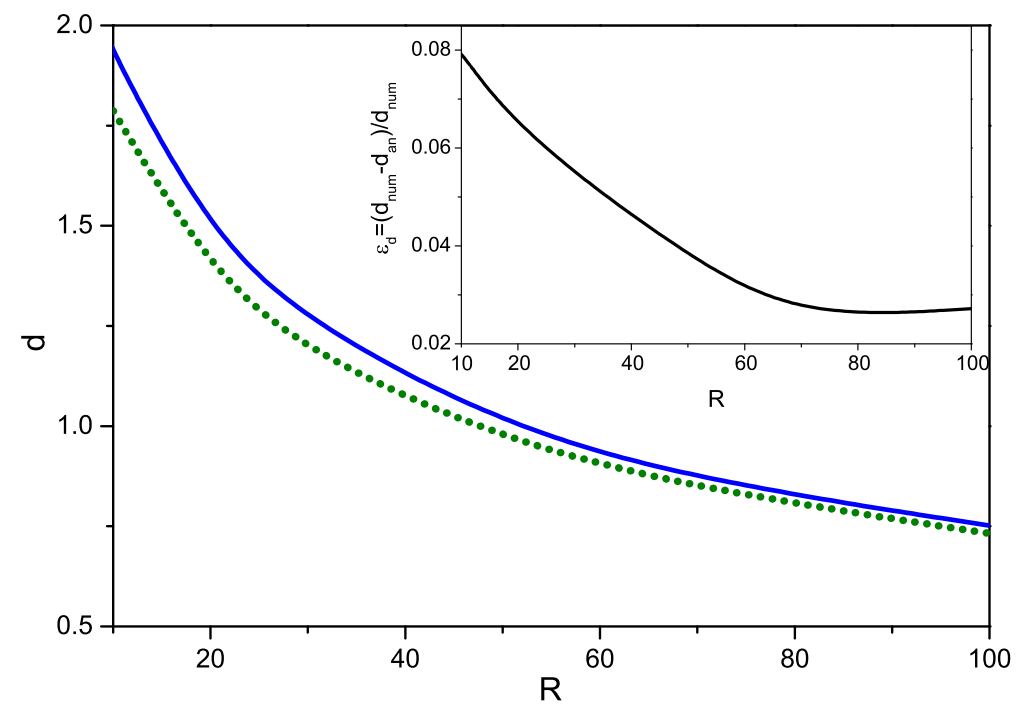

Figure 4.11: Optimum cell size $-d_{\text {opt }}$ as a result of numerical simulations $d_{\text {num }}$ (shown by solid blue line) is plotted alongside analytical approximation given by Eq. $4.34 d_{a n}$ (shown by dotted green line). The inset shows approximation error defined as $\varepsilon_{d}=\left(d_{\text {num }}-\right.$ $\left.d_{a n}\right) / d_{\text {num }}$
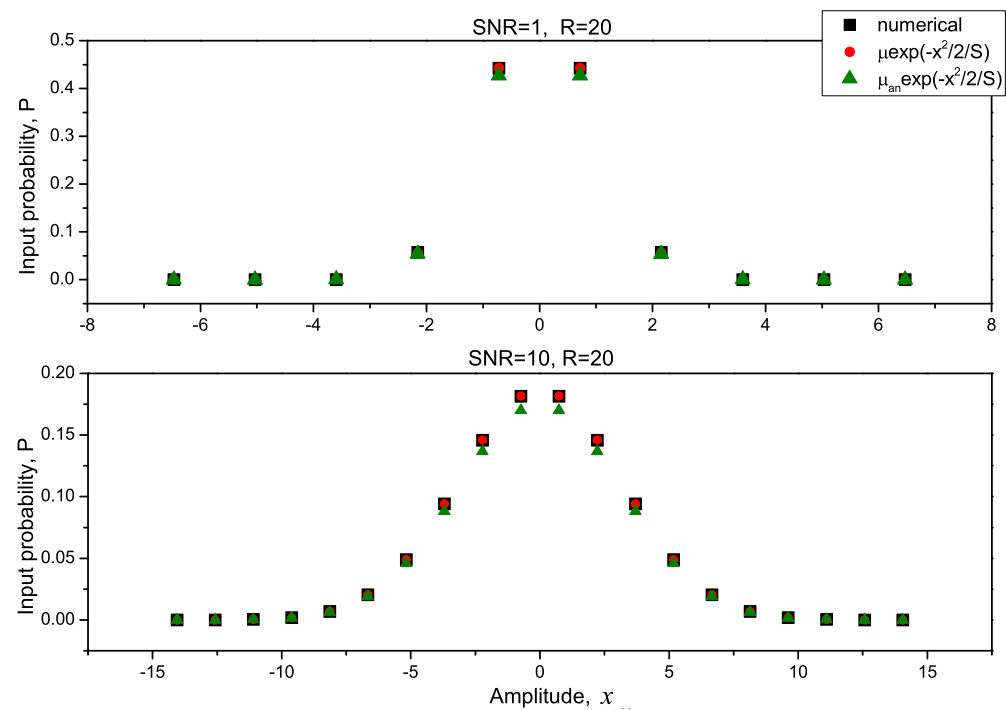

Figure 4.12: Optimum input probability $-P_{k}$ as a result of numerical simulations (shown by black squares) is plotted alongside Gaussian approximation given by $P_{k}=$ $\mu \exp \left[-x_{k}^{2} /(2 S)\right]$ (shown by red circles), where $\mu$ is normalization constant chosen to satisfy condition $\sum_{k=1 . . K} P_{k}=1$. The analytical approximation $P_{k}=d \sqrt{2 \pi S}^{-1} \exp \left[-x_{k}^{2} /(2 S)\right]$ (with $\mu_{a n}=d \sqrt{2 \pi S}^{-1}$ ) is depicted by green triangles. 

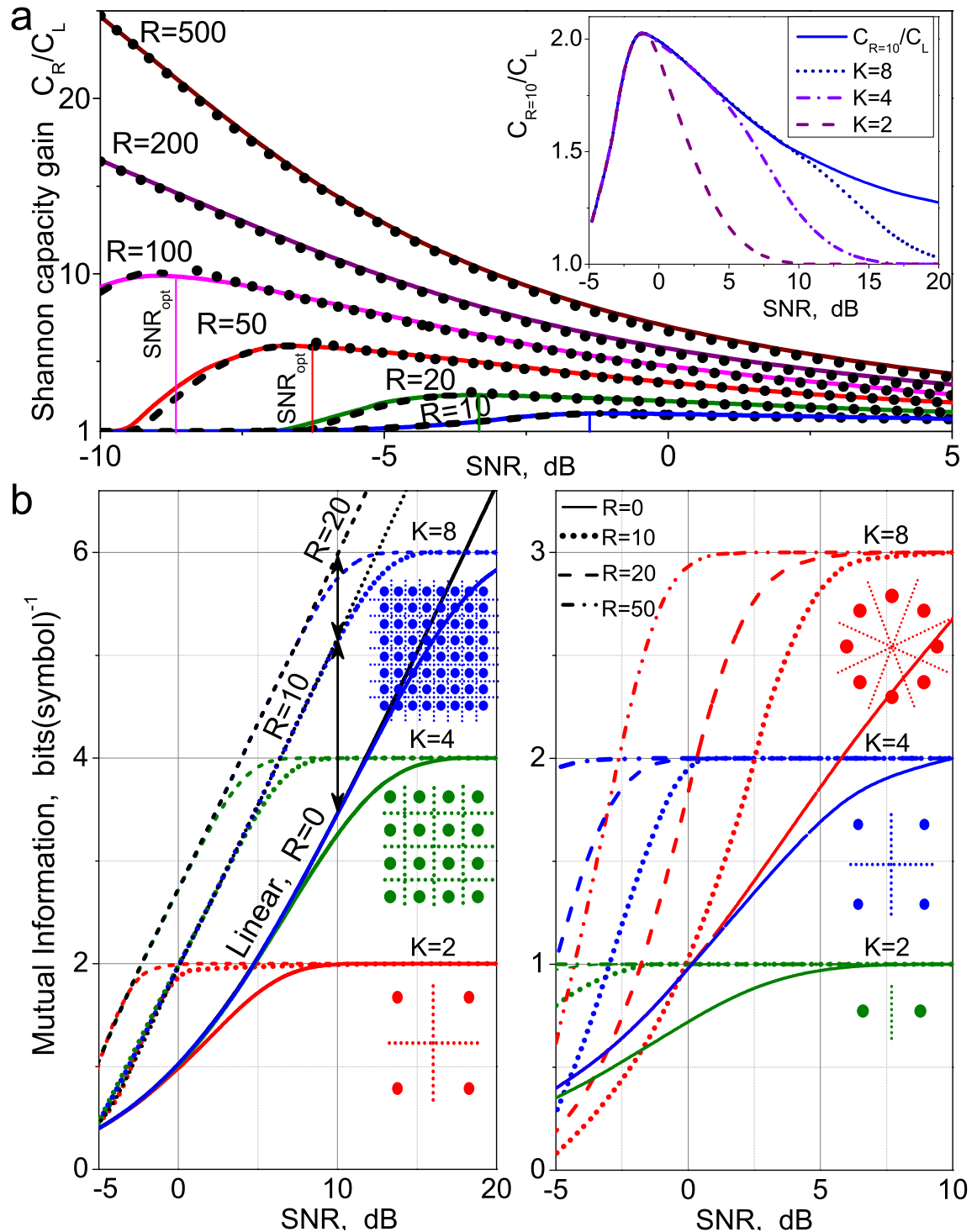

Figure 4.13: Regeneration limit. - a) Gain, the regenerative capacity ratio to the Shannon formula $C_{L}=\log _{2}(1+S N R)$, for the different number of regenerators. The analytical results demonstrating an excellent agreement with numerics are shown by black (dashdotted Eq.4.16 for $S N R \cdot R \ll 1$ and dotted using Eq.4.35 for $S N R \cdot R \gg 1$ ) lines. The inset shows mutual information gain for two-dimensional $(n=2) K^{2}$ - rectangular constellations. b) Capacity and mutual information for discrete two-dimensional $K^{2}$ - rectangular QAM (left panel) and $K$ - points in the ring $K$-PSK (right panel) alphabets. The arrows show the constant capacity increase. Constellations and associated attraction regions are shown. 


\subsection{Upper bound of regeneration efficiency - capacity of the channel with ideal} regenerators

As a result of numerical optimization, the input probability was found to be a discrete analogue of Gaussian probability (see Fig. 4.12). Thus, the maximum capacity gain due to regeneration (that is, the maximum regeneration efficiency) is observed for the binary channel. This emphasizes simple binary channel efficiency. The capacity gain reflects the trade-off between the system complexity and improvement. As the number of regenerators increases, the capacity gain peak shifts to smaller SNR. Therefore, employing a low SNR regime and using regeneration, one can achieve high transmission performance. Moreover, at this SNR interval, destructive nonlinearity impact is negligible, which might be important for practical design consideration. The minimum SNR value, when $d_{o p t}$ is achieved, defines the maximum capacity ratio to its linear analogue, that is, $S N R_{o p t}=d_{o p t}^{2} / 4 N$. At this SNR value, both analytic formula Eqs. 4.16 and 4.35 can be interpolated to describe capacity at the full range of SNR.

The analytical approximations Eqs. 4.16 (for $S N R \cdot R \ll 1$, shown by dashed lines) and 4.35 (for $S N R \cdot R \gg 1$, shown by dotted lines) depicted in Fig. 4.13 by black lines demonstrate an excellent agreement with the result of numerical computations of the capacity for different number of regenerators (colored solid lines). The figure demonstrates that regeneration allows substantially reducing noise impairments and, consequently, achieving capacity above the linear Shannon capacity (Shannon capacity of linear AWGN channel). The maximum system improvement is observed at low SNR values $-S N R_{\text {opt }}$ shown by vertical lines.

Similar to the rectangular case, the analysis was extended to ring formats with conditional probabilities defined through the matrix elements:

$$
P\left(y=x_{k} \mid x_{l}\right)=\int_{S_{k}} d x^{\prime} p_{G}\left(x^{\prime} \mid x_{l}\right)=W_{k l}, \quad p_{G}\left(x^{\prime} \mid x_{l}\right)=\frac{1}{\pi N / R} \exp \left(-\frac{\left(x^{\prime}-x_{l}\right)^{2}}{N / R}\right)
$$

For $K$-points placed in a single ring of radius $\sqrt{S}$ (phase-shift-keying format) the transition matrix is defined as follows:

$$
W_{k l}=\int_{0}^{\infty} d r \int_{2 \pi k / K}^{2 \pi k / K+\pi / K} d \varphi \frac{r}{\pi N / R} \exp \left[-\frac{r^{2}}{N / R}-\frac{S}{N / R}+\frac{2 r \sqrt{S}}{N / R} \cos \left(\varphi-\frac{2 \pi l}{K}\right)\right]
$$

Figure $4.13 \mathrm{~b}$ shows mutual information for rectangular quadrature amplitude modulation (QAM) in the left panel and ring phase-shift-keying (PSK) in the right panel. Here, an optimization role is demonstrated: the constellation choice (defined by the 
SNR value and the number of the in-line regenerators) results in a capacity dramatic gain, whereas the non-optimal format results in nonlinear capacity lower than the respective linear one.

Note, the channel capacity depends on two parameters only: SNR and the number of ideal regenerators $R$. Since the result of the nonlinear transformation is the noise squeezing defined by the value of $R$, the system behavior is governed by SNR, in contrast to conventional Kerr-nonlinear problem, where the system performance depends non-trivially on $S$ and $N$ and cannot be captured by single parameter $S N R=S / N$. It was verified numerically that fixing the value of $N$ and varying signal power $S$ or fixing $S$ and varying noise power $N$ gives the same capacity results for the considered channel.

An important feature of the channel is that capacity gain is achieved by regenerative transformations without signal amplification. An ideal regenerator assigns distorted points to the closest alphabet point. Therefore, if a signal, distorted by noise with variance $N_{m}$, had power $S+N_{m}$, the regenerated signal will have power equal to the undistorted signal $S$.

The ideal regenerator fully suppresses distortion within the regenerative region $S_{k}$ and assigns the distorted point $x^{\prime}$ to the original alphabet point $x_{k}$. So, an error will occur only if the distorted point $x^{\prime}$ is diffused out of the regenerative region, when distortion is larger than $\| S_{k}|| / 2$. Therefore, the ideal regenerator (given by the step-wise transfer function) has the highest efficiency regeneration and the Shannon capacity of such a channel is the highest that can be achieved - this we refer to as the regenerative Shannon limit.

In practice, there are a variety of all-optical regenerators characterized by smooth transfer functions, which enable approaching an ideal step-wise function (see Fig.4.9). In the next section, we demonstrate capacity calculations for such systems on the example of the regenerative Fourier transform model. 


\subsection{Capacity calculation for regenerative mapping}

The signal evolution in such systems (see Fig. 4.1) can be presented by the stochastic map - a discrete version of the Langevin equation for stochastic processes:

$$
Y_{m}=T\left(Y_{m-1}\right)+\eta_{m}, \quad m=1, \ldots, R+1, \quad Y_{0}=x
$$

As in previous sections, notations $x_{k}$ and $Y_{m}$ (and its deterministic outcome $y_{m}$ ) are used for $k$-th alphabet point and signal at $m$-th span correspondingly. Again, twodimensional problems can be considered as two independent lattices with real variables $x$ and $y$. Here $m$ is the discrete spatial index and $T$ is the transfer function of the regenerative regenerator. The term $\eta_{m}$ models the Gaussian noise with zero mean and the variance given by $N_{m}$ added at $m$-th node; further, we assume regenerators to be placed equidistantly, so for all $m: N_{m}=N_{m+1}=N /(R+1)$, where $N$ is total power of noise added to the signal during transmission.

The conditional pdf for the output at $m$-th node for each quadrature $y_{m}$ given the input $y_{m-1}$ is found as

$$
p\left(y_{m} \mid y_{m-1}\right)=\frac{1}{\sqrt{2 \pi N_{m}}} \exp \left[-\frac{\left(y_{m}-T\left(y_{m-1}\right)\right)^{2}}{2 N_{m}}\right]
$$

Because of the Markovian property of the process, the conditional pdf of the received signal after propagation through $R$ links, $y_{R}$, given the input, $x$, is expressed by a product of single-step conditional probabilities

$$
p\left(y_{R+1} \mid y_{0}=x_{k}\right)=\int d y_{R} . . d y_{1} p\left(y_{R} \mid y_{R-1}\right) \ldots p\left(y_{1} \mid y_{0}=x_{k}\right)
$$

Consequently, when for any $m: N_{m}=N_{m+1}=N_{1}=$ const, the conditional pdf can be expressed through an Onsager-Machlup function or action of the path given by

$$
\mathcal{S}=\Sigma_{m=1}^{R+1} \frac{1}{\sqrt{2 \pi N_{m}}} \frac{\left(y_{m}-T\left(y_{m-1}\right)\right)^{2}}{2 N_{m}}
$$

as follows

$$
p\left(y_{R+1} \mid y_{0}\right)=\int d y_{R} . d y_{1} e^{-\mathcal{S}}
$$




\subsubsection{Numerical calculation of the Shannon capacity of RFT chan- nel}

Substituting transfer function of RFT: $T(y)=y+a \sin (b y)$ we obtain the conditional pdf for the channel output $y_{R+1}$ given input - alphabet point $x_{k}$ :

$$
p\left(y_{R+1} \mid y_{0}=x_{k}\right)=\int \prod_{m=1}^{R} d y_{m} \frac{1}{\sqrt{2 \pi N_{m}}} e^{-\left(y_{m+1}-y_{m}-a \sin \left(b y_{m}\right)\right)^{2} / 2 N_{m}}
$$

This expression calculated numerically (using trapezoidal integration with step 0.005 ) is plotted in Fig. 4.14 for the same power of added noise $N=\sum_{m=1}^{R+1} N_{m}$. The conditional pdf demonstrates that the regenerator acts as a quantizer by attracting signal points to the alphabet - one can observe peaks formed around the alphabet points. The alphabet is constructed in accordance with the proposed optimization procedure of Eq. 4.1, which for the RFT case defines $x_{k}=\pi(-K+1+2 k) / b$, with $\left.k=1 . . K\right)$. Also, one can see that for the optimal parameters choice, the noise squeezing effect is more pronounced (compare suboptimal case plotted in Fig. 4.14a with optimal in Fig. 4.14b), whereas, since suboptimal case peaks are wider, the interference between them is more clear (compare Fig. 4.14a with inset in Fig. 4.14b). This illustrates the difference between the linear and considered regenerative nonlinear channels: if the distortion is larger than the cell size, then the diffused point will be attracted to the wrong alphabet point (not the same as the original [before distortion] alphabet point). This stresses the discreteness of the problem: the cell size cannot be arbitrarily small (this is in contrast to the linear channel) and the optimum distribution is discrete. While the noise power $N$ was fixed (here $N=1$ ), placing alphabet points closer to each other (that is, decreasing cell size) resulted in higher interference between the peaks and reduction of the regenerative effect (compare Fig. 4.14b with tighter packing in Fig. $4.14 \mathrm{c}$ ). This demonstrates the importance of optimization over the cell size and the necessity to adapting it to the noise power and the number of regenerators. Further decreasing the cell size results in the smoothing of peaks and at $d \rightarrow 0$, the regenerative effect vanishes. Obviously, increasing the number of regenerators leads to higher regeneration efficiency (Fig. 4.14). 


\subsection{Capacity calculation for regenerative mapping}
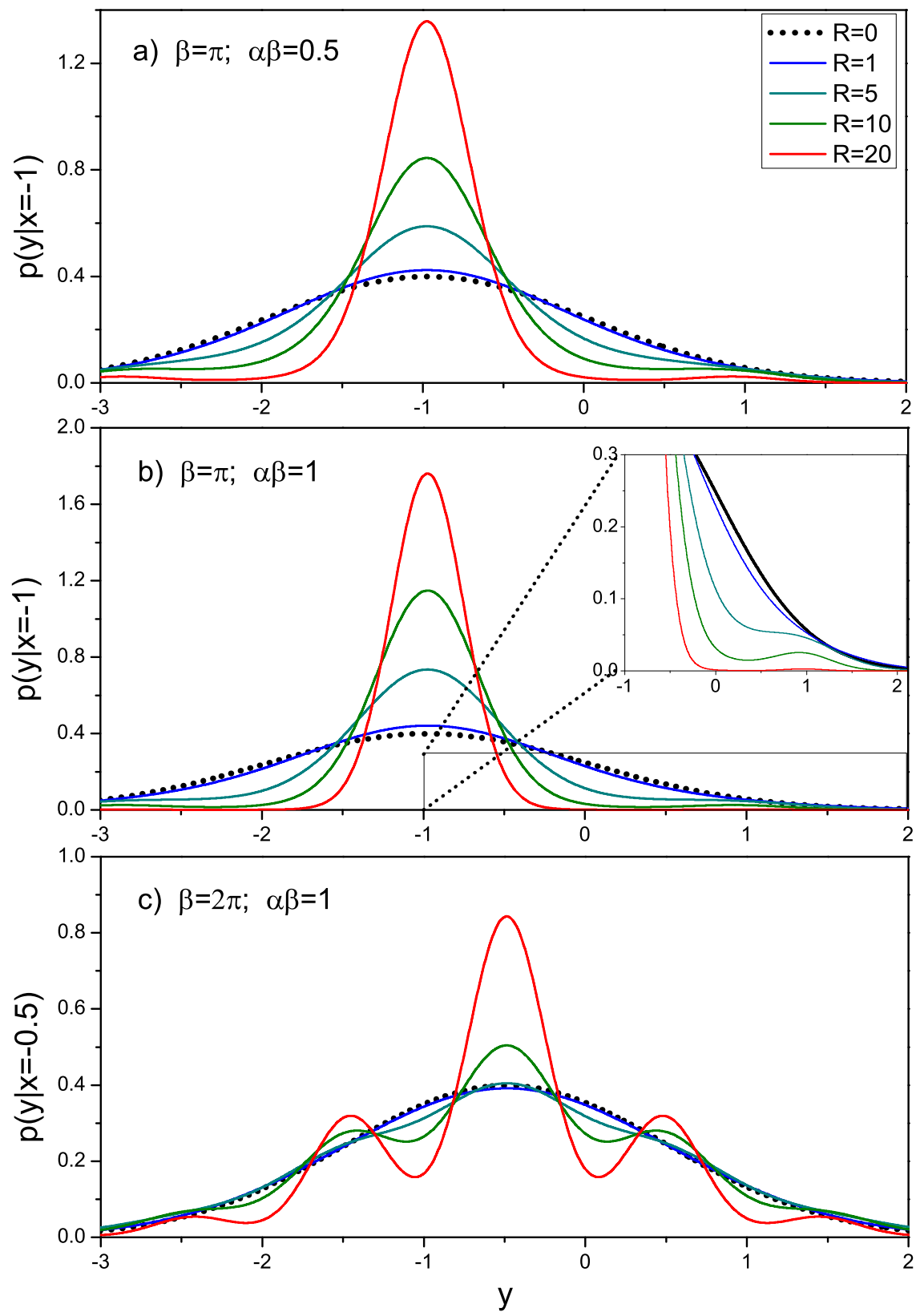

Figure 4.14: Conditional pdf. - $p\left(y_{R+1} \mid x\right)$ for a different number of regenerators a) for suboptimal $a b=0.5$ and b) optimal $a b=1$ parameters choice and for the same power of total noise $N=1$. Regenerative transformation results in the formation of peaks around alphabet points given by the regenerative mapping procedure. Increasing the number of regenerators results in more narrow peaks and higher noise squeezing, whereas suboptimal parameters choice, though it results in significant noise reduction, has worse performance than the optimal parameters choice $a b=1$. Decreasing the cell size from $d=2$ (panels $\mathrm{a}-\mathrm{b}$ ) to $d=1$ (panel c) results in worse system performance. It shows that the channel is essentially discrete and the cell size needs to be optimized. Increasing the number of regenerators improves regeneration efficiency. 


\subsection{Capacity calculation for regenerative mapping}

\subsubsection{Impact of parameter $b$}

Using conditional pdf, one can calculate the Shannon capacity of the channel. Regenerative mapping techniques enable finding amplitude distribution optimal for a given regenerative nonlinearity. In particular case of RFT, the alphabet is given as $x_{k}=\pi(-K+1+2 k) / b$, where $k=1 . . K$ is an index of an alphabet point. We choose the alphabet according to regenerative mapping technique, and as distortion around these points is suppressed due to regeneration, using a different set of $x_{k}$ will result in increase of distortion due to applied nonlinear transformation. Effectively, regenerative mapping technique optimizes modulation according to the given nonlinearity. So, the optimal amplitude distribution is equidistant with the cell size defined by parameter $b$.

The program for numerical simulations was written in Matlab using a built-in SQP algorithm with parameters of the function set to standard termination tolerance on the function value was set to be $10^{-6}$ (other parameters are: the relative first-order optimality measure is less than $10^{-6}$ and the relative maximum constraint violation is less than $10^{-6}$ ). The initial search point was chosen to be equiprobable distribution (it was verified that the change of initial values does not affect optimization result). The constraints were $\sum P_{k}\left|x_{k}\right|^{2}=S$ and $\sum P_{k}=1$. The input parameters were: $S N R=S / N$ and $R$. The number of signal points (constellation size $K$ ) was varied until its increase does not affect the capacity calculations by more than $10^{-4}$. The conditional pdf Eq. 4.44 was calculated numerically using trapezoidal integration with step 0.005 (it was checked that the step size 0.001 did not affect the capacity calculations by more than $\left.10^{-4}\right)$.

The optimal input pmf (see Fig. 4.15) is found to be a discrete analogue of Gaussian distribution: $P_{k} \sim e^{-x_{k}^{2} / 2 S}$. The value of parameter $b$ was varied and in Fig. 4.15 results for two cases $b=\pi$ and $b=2 \pi$ are plotted. As the cell size is reduced $x_{k+1}-x_{k}=$ $2 \pi / b$, the constellation size $K$ is increased (compare panels a and b in Fig. 4.15). The corresponding constrained capacity (MI in Eq. 2.32 optimized over input probability distribution for a given modulation format) for fixed values $b=\pi, 2 \pi, 3 \pi$. One can see the impact of the parameter $b$ on the constrained capacity. So, to achieve Shannon capacity of the channel - maximum of constrained capacity, parameter $b$ needs to be optimized. 


\subsection{Capacity calculation for regenerative mapping}

The Fig. 4.16 demonstrates how the choice of parameter $b$ affects the constrained capacity, and how the other parameter $a$ was related to the parameter $b$ as $a b=1$. If $b=\pi$, the constrained capacity is higher than the linear Shannon limit (and the capacity gain is higher than 1$)$. If the cell size is reduced $(=2 \pi / b)$, though the constellation size is increased (see pmf in Fig. 4.15), the constrained capacity becomes smaller, for $b=2 \pi$ the constrained capacity is approximately equal to the linear Shannon limit and the gain, though still above unity, is roughly equal to one. So, decreasing the cell size below the optimal value, reduces the regenerative effect. Further, decrease of the cell size $b=3 \pi$ degrades the constrained capacity below the linear Shannon limit - regeneration has negative impact: the cell size is so small (compared to the noise variance), that there is high probability that the distorted point will be attracted to the wrong alphabet point and a regenerator will only increase distortion. This demonstrates that the optimal distribution is discrete and the cell size (and, consequently, parameter b) needs to be optimized.

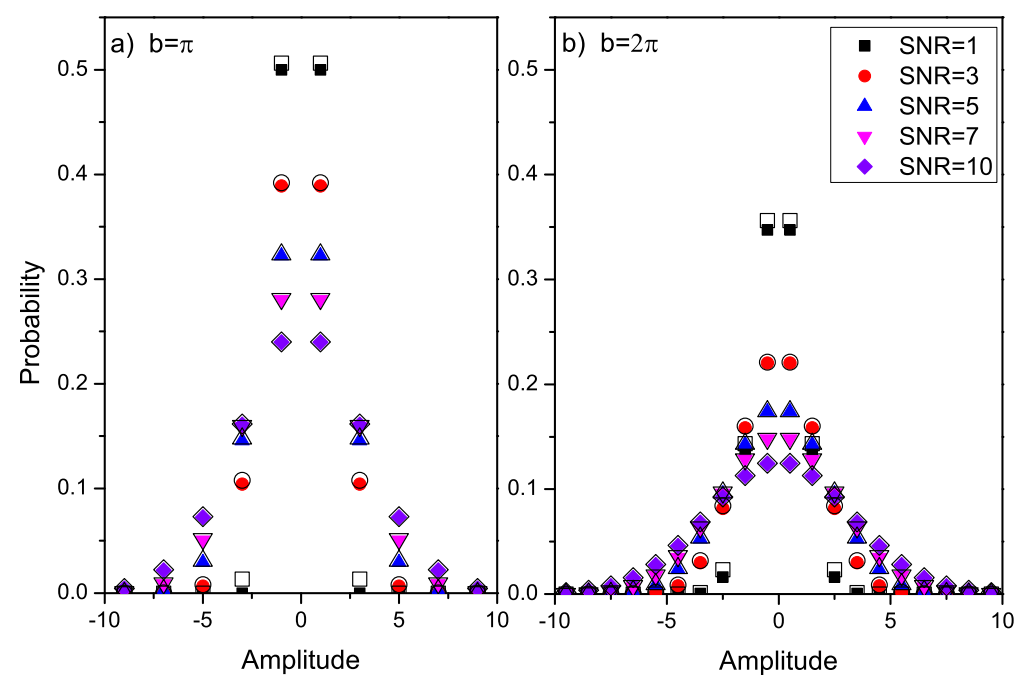

Figure 4.15: Optimum input pmf - $P_{k}$ as a result of numerical simulations as a function of SNR for $R=10$ (shown by colored filled symbols) coincides with a discrete analogue of Gaussian distribution: $\sim e^{-x_{k}^{2} / 2 S}$ (shown by black unfilled symbols) (here $N=1$ was assumed). We plot here results for two cases $b=\pi$ and $b=2 \pi$. 


\subsection{Capacity calculation for regenerative mapping}

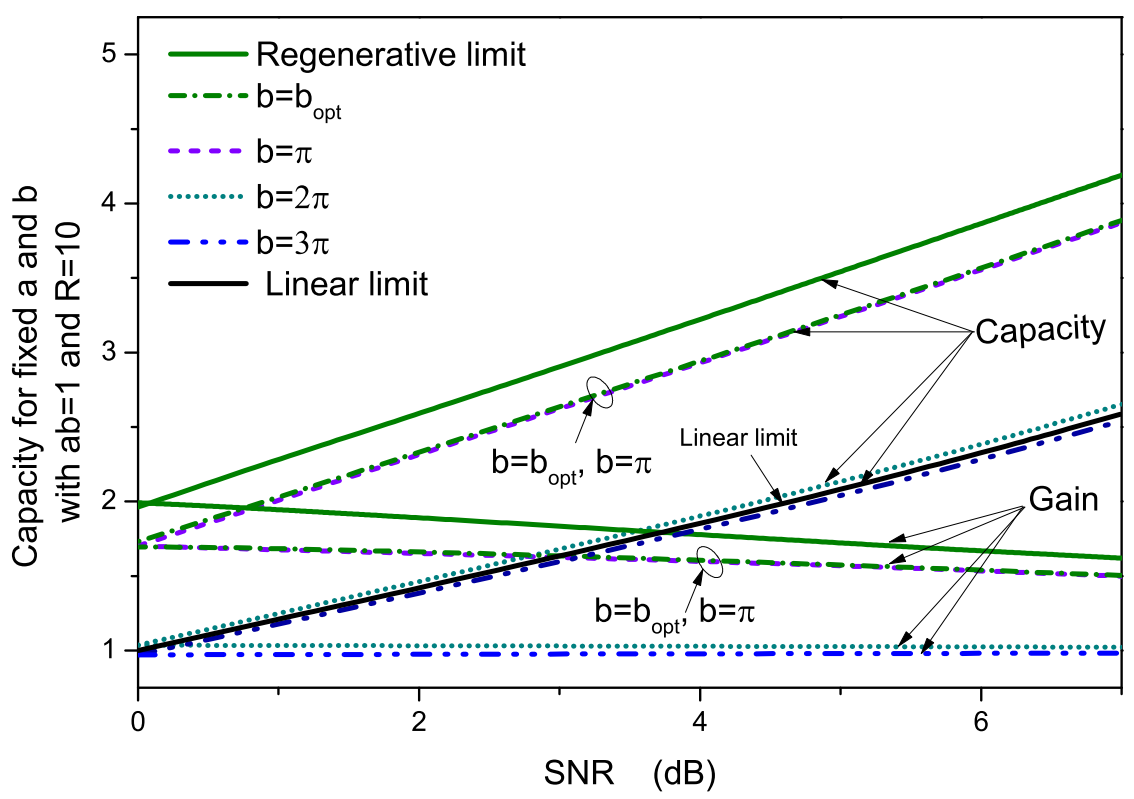

Figure 4.16: Constrained capacity of the two-dimensional RFT channel and the corresponding gain (ratio to the linear Shannon limit $\log _{2}(1+S N R)$ ) - optimized over probability distributions for a given modulation format: equidistant modulation with the cell size $2 \pi / b$ for $b=\pi, 2 \pi, 3 \pi$ for a fixed number of RFT regenerators $R=10$. Shannon capacity of the RFT channel (optimized over modulation and probability distribution $b=b_{\text {opt }}$ ) and Shannon capacity of a channel with ideal regenerators - regenerative limit are plotted for comparison. The value $b=\pi$ gives constrained capacity in the vicinity of the Shannon capacity, whereas $b=2 \pi$ and $b=3 \pi$ (modulations with smaller cell size) give significantly smaller constrained capacity, close to the linear case, with the Shannon capacity of linear AWGN channel having the same SNR. This illustrates that the optimal distribution is discrete. This contrasts with the linear channel, where optimal distribution is continuous. 


\subsection{Capacity calculation for regenerative mapping}

\subsubsection{Optimization of parameter $b$}

It was shown (from the analysis of conditional pdf) that the cell size needs to be optimized as a function of SNR and a number of regenerators $R$. Therefore, we optimize the problem over parameter $b$, which defines amplitude distribution. Optimization over parameter $b$ was conducted by calculating constrained capacity (optimization over input pmfs) for different values of $b$, the maximum was found with function tolerance, that is, optimization was stopped when the difference between the calculated values of the constrained capacities was less than $10^{-4}$.

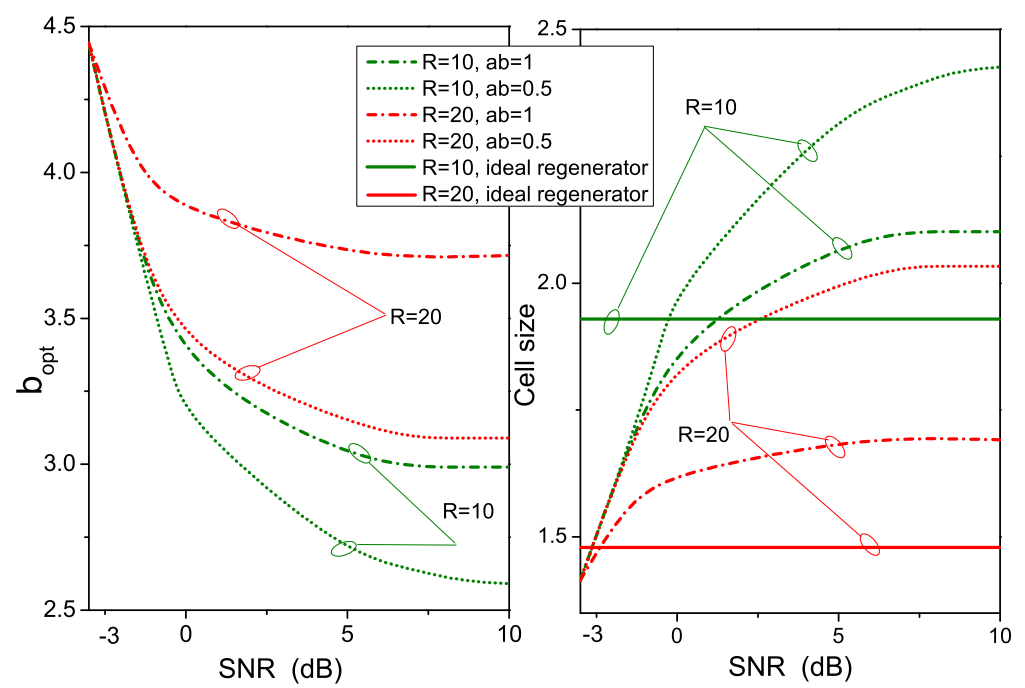

Figure 4.17: Optimum value of a) parameter $b$ and b) cell size versus SNR - calculated as a result of numerical optimization for different number of regenerators in-line $R=10$ and 20 (green and red curves) and different parameters relation: suboptimal $a b=0.5$ (dotted) and optimal $a b=1$ (dash-dotted). Optimum cell size in case of the channel with ideal regenerators (see Fig. 4.11) is plotted alongside by solid lines.

The numerically calculated optimal values of parameter $b$ and the corresponding cell size $=2 \pi / b$ are plotted in Fig. 4.17. One can see that the optimum cell size decreases when regeneration is more pronounced, that is, when the number of regenerators is increased or when the parameters are closer to the optimum $\left(T^{\prime}(x)=0\right.$ when $a b=1)$. In other words, when regeneration is stronger, noise is suppressed more and one can operate with smaller cell size - tighter packing. This directly reflects in capacity. 


\subsection{Capacity calculation for regenerative mapping}

\subsubsection{Impact of parameter $a$}

Additional parameter $a$ governs the strength of regeneration. In accordance with stability requirement of regenerative mapping technique: $\left|T^{\prime}(x)\right|<1$, it follows that if $a=2 / b$ one can observe plateau formed around the alphabet points; in this case, noise is completely suppressed within the plateaus (this is the best choice of parameter values). Thus, the parameter $b$ also defines the value of parameter $a$.

As was noted before, though being the best choice, plateau is not necessary. If $a<1 / b$, one can still observe a regenerative effect. We demonstrate this by calculating capacity for the suboptimal choice $a=1 / 2 b$. When $a=0$ there is no regeneration linear AWGN channel.

\subsubsection{Shannon capacity of the RFT channel}

Optimizing over parameter $b$ and input pmfs we found maximum of MI over the set of $\left\{P_{k}, b\right\}-$ Shannon capacity of the RFT channel for two cases $a=1 / b$ and $a=1 / 2 b$ Fig.4.18.

The proposed RFT channel demonstrates visible capacity gain over the Shannon capacity of the linear AWGN channel. Here we demonstrate that though TF with plateau is the most efficient, nevertheless, it is not necessary for regeneration. One can see that suboptimal parameter values also provide a capacity increase. The set of conditions given by Eq.4.1 defines optimization and design rules for implementation of such nonlinear regenerative channels. Optimal input distribution is plotted in Fig.4.19. The optimal distribution is discrete with equidistantly placed alphabet points and the pmf is a discrete analogue of Gaussian distribution: $\exp \left(-x_{k}^{2} / 2 S\right)$.

The results illustrate that by suppression of signal distortions, one can achieve Shannon capacity higher than a system without regenerators operating with the same signal launch power and with the same power of noise added to the signal during transmission. In the high SNR limit, the Shannon capacity of the considered channel tends asymptotically to the regenerative Shannon limit, which was calculated analytically in Eq. 4.35. 


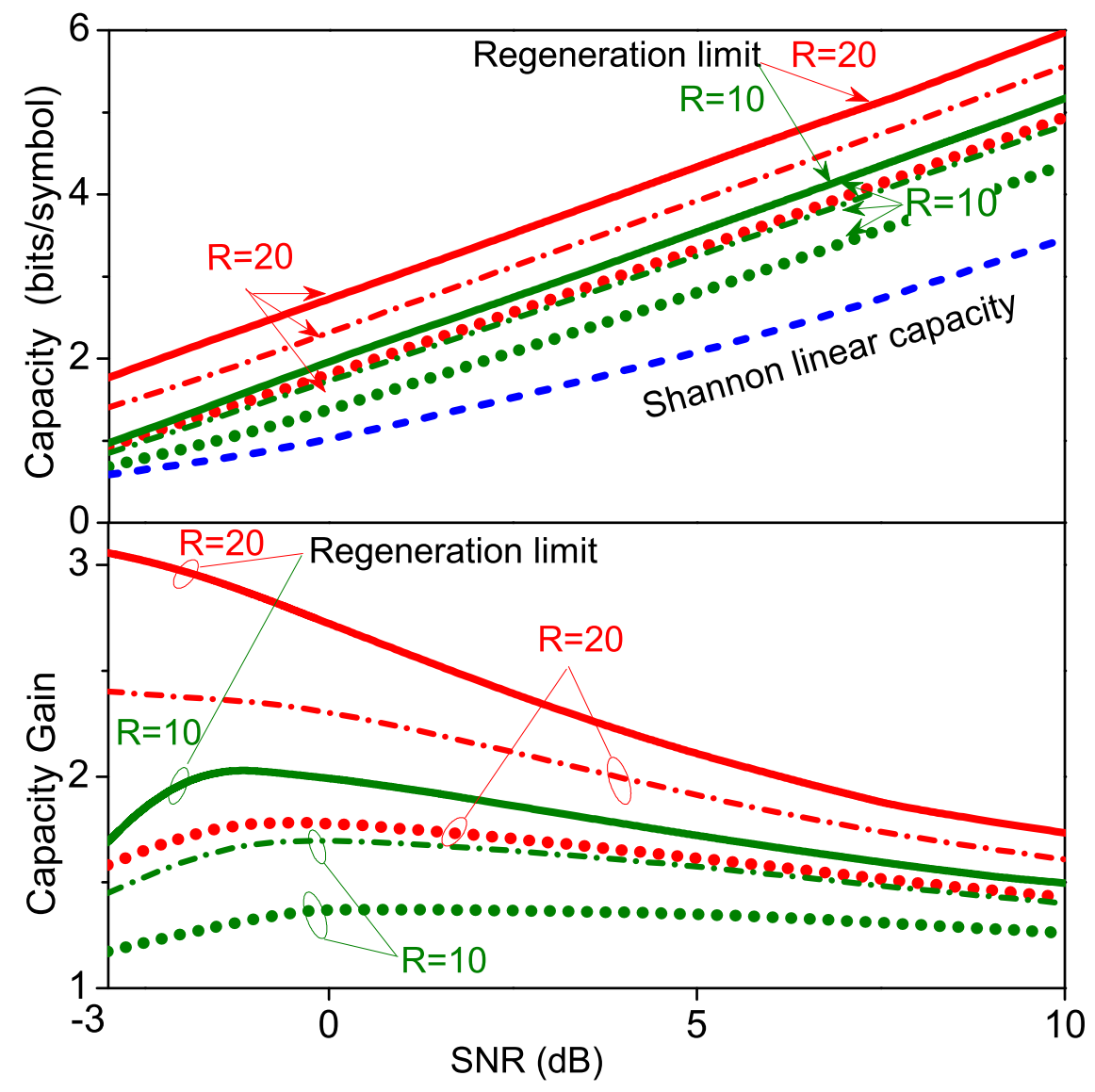

Figure 4.18: Shannon capacity of the RFT channel and the corresponding capacity gain - calculated as a result of numerical optimization for different number of regenerators in-line $R=10,20$ (green and red curves) and different parameters relation: suboptimal $a b=0.5$ (dotted) and optimal $a b=1$ (dash-dotted). Shannon capacity of the channel with ideal regenerators - regenerative Shannon limit plotted alongside by solid curves. 


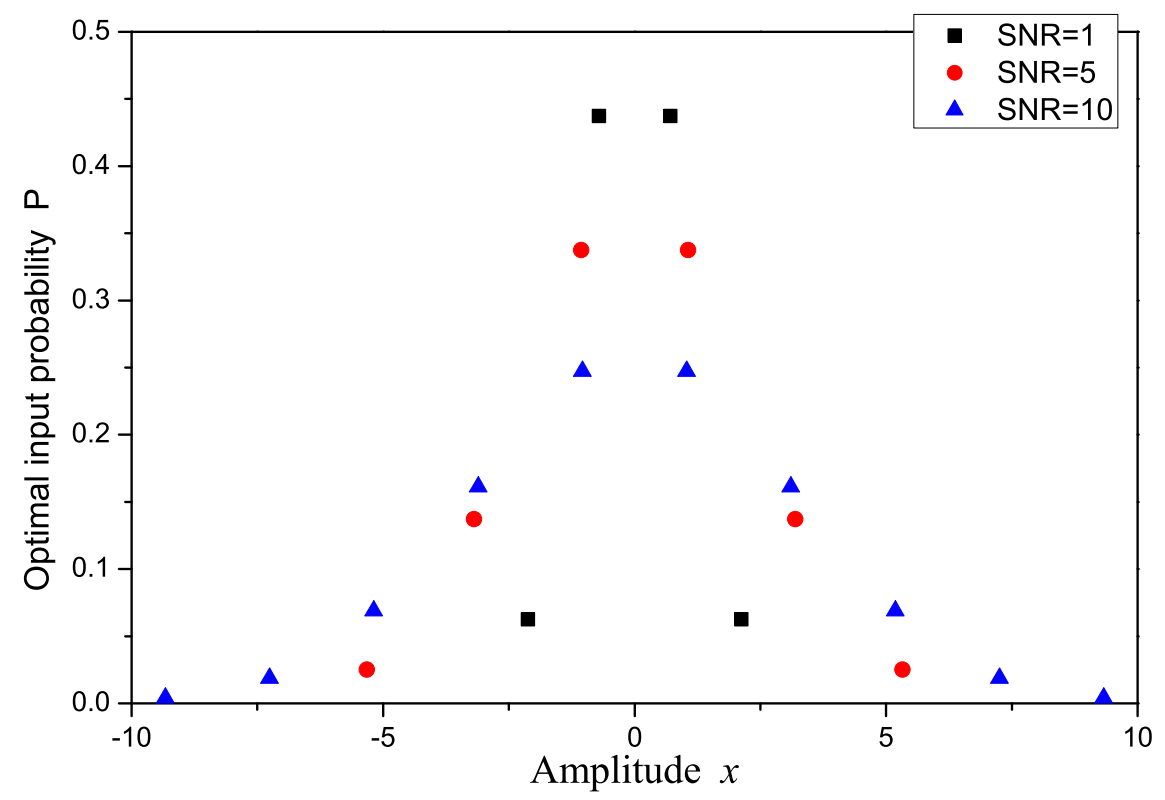

Figure 4.19: Optimal input probability -

\subsubsection{Upper bound of capacity for the RFT mapping}

The conditional pdf for the sine-regeneratoring model is given by Eq.4.44:

$p\left(y_{R+1} \mid x_{k}\right)=\int \prod_{m=1}^{R} d y_{m} \frac{1}{\sqrt{2 \pi N_{m}}} e^{-\left(y_{m+1}-y_{m}-a \sin \left(b y_{m}\right)\right)^{2} / 2 N_{m}}, y_{0}=x_{k}, N_{m}=N /(R+1)$

We can reduce the problem to the previously considered in Section 4.4.1. Let us start by considering the conditional probability for the first link:

$$
p\left(y_{1} \mid x_{k}\right)=\frac{1}{\sqrt{2 \pi N_{m}}} \exp \left(-\frac{\left(y_{1}-x_{k}\right)^{2}}{2 N_{m}}\right)
$$

Let us represent it as a sum of probabilities through the decision boundaries, namely as a sum of a distorted point $y_{l}$ to be in the decision region $S_{l}$ of the point $x_{l}$, this is the same as $W_{l k}$ in Eq. 4.7:

$$
\begin{aligned}
p\left(y_{1} \mid x_{k}\right) & =\left.\sum_{l} P\left(y_{1} \in S_{l} \mid x_{k}\right) \frac{1}{\sqrt{2 \pi N_{m}}} \exp \left(-\frac{\left(y_{1}-x_{k}\right)^{2}}{2 N_{m}}\right)\right|_{y_{1} \in S_{l}} \\
& =\left.\sum_{l} W_{l k} \frac{1}{\sqrt{2 \pi N_{m}}} \exp \left(-\frac{\left(y_{1}-x_{k}\right)^{2}}{2 N_{m}}\right)\right|_{y_{1} \in S_{l}}
\end{aligned}
$$




\subsection{Capacity calculation for regenerative mapping}

Where we used $P\left(y_{1} \in S_{l} \mid x_{k}\right)=W_{l k}$ defined in Eq. 4.7. In the ideal regenerator case, the conditional pdf $p\left(y_{1} \mid x_{k}\right)$ was thoroughly defined by the transition matrix $\mathbf{W}$ (since after the ideal regenerator $y_{1}=x_{l}$ ). However, the regenerator with smooth transfer function does not assign the distorted point $y_{1}$ to $x_{l}$, but attracts it, so $y_{1}$ becomes closer but not equal to $x_{l}$. Thus, the conditional pdf $p\left(y_{1} \mid x_{k}\right)$ has a continuous part. The representation of $p\left(y_{1} \mid x_{k}\right)$ as a sum over decision boundaries $S_{l}$ allows us to extract the small parameter $\varepsilon=y_{1}-x_{l}$.

Let us proceed with the second link:

$$
\begin{gathered}
p\left(y_{2} \mid x_{k}\right)=\int d y_{1} p\left(y_{2} \mid y_{1}\right) p\left(y_{1} \mid x_{k}\right)= \\
\frac{1}{2 \pi N_{m}} \int d y_{1} \exp \left(-\frac{\left(y_{1}-x_{k}\right)^{2}}{2 N_{m}}-\frac{\left(y_{2}-y_{1}-a \sin \left(b y_{1}\right)\right)^{2}}{2 N_{m}}\right)
\end{gathered}
$$

Then we substitute $p\left(y_{1} \mid x_{k}\right)$ as in Eq. 4.47 using the small parameter $\varepsilon=y_{1}-x_{l}$ :

$$
p\left(y_{2} \mid x_{k}\right)=\sum_{l} W_{l k} \int d \varepsilon p\left(y_{2} \mid x_{l}+\varepsilon+a \sin \left[b\left(x_{l}+\varepsilon\right)\right]\right) \frac{1}{\sqrt{2 \pi N_{m}}} \exp \left(-\frac{\left(\varepsilon-x_{k}+x_{l}\right)^{2}}{2 N_{m}}\right)
$$

under assumptions of $\varepsilon$ to be small and using the definition of the alphabet points, namely $x_{k}=\pi(-K+1+2 k) / b$ where $k=1 \ldots K$, and expanding the sine function in series over $\varepsilon$ we arrive at $\lim _{\varepsilon \rightarrow 0} y_{2}-y_{1}=\lim _{\varepsilon \rightarrow 0} y_{2}-x_{l}-\varepsilon-a \sin \left(b\left(x_{l}+\varepsilon\right)\right)=y_{2}-$ $x_{l}-\varepsilon(1-\rho)+O\left(\varepsilon^{2}\right)$, where $\rho=a b$. Thus, the conditional pdf $P\left(y_{2} \mid x_{l}+\varepsilon+a \sin \left[b\left(x_{l}+\right.\right.\right.$ $\varepsilon)])$ can be simplified to $p\left(y_{2} \mid x_{l}+(1-\rho) \varepsilon\right)$, which reflects in:

$$
p\left(y_{2} \mid x_{k}\right)=\sum_{l} W_{l k} \frac{1}{2 \pi N_{m}} \int d \varepsilon \exp \left(-\frac{\left(x_{k}-x_{l}-\varepsilon\right)^{2}}{2 N_{m}}-\frac{\left(y_{2}-x_{l}-\varepsilon(1-\rho)\right)^{2}}{2 N_{m}}\right), \rho=a b
$$

Integrating we obtain

$$
p\left(y_{2} \mid x_{k}\right)=\sum_{l=1}^{K} W_{l k} \frac{1}{\sqrt{2 \pi N_{m}\left(1+(1-\rho)^{2}\right)}} \exp \left(-\frac{\left(y_{2}-x_{l}+\rho\left(x_{k}-x_{l}\right)\right)^{2}}{2 N_{m}\left(1+(1-\rho)^{2}\right)}\right)
$$

Note, after the transformation, the noise is reduced, so the residual noise at second link is: $N_{2}^{\text {res }}=N_{m}\left(1+(1-\rho)^{2}\right)=N\left(1+(1-\rho)^{2}\right) /(R+1)$. Further, one can use the same procedure (rewrite conditional pdf of each link through the sum to extract small 


\subsection{Capacity calculation for regenerative mapping}

parameter and then expand sine function over it). So, after repeating the procedure multiple times, the conditional pdf for the final link will have the form:

$$
p\left(y_{R+1} \mid x_{k}\right)=\sum_{l, l^{\prime}, l^{\prime \prime} . .} W_{k l} W_{l l^{\prime}} \cdot \frac{1}{\sqrt{2 \pi N_{R+1}^{r e s}}} \exp \left(-\frac{\left(y_{R+1}-f\left(x_{k}, x_{l}, x_{l^{\prime}}, \ldots\right)\right)^{2}}{2 N_{R+1}^{r e s}}\right)
$$

where $f$ is a function of alphabet points $f\left(x_{k}, x_{l}, x_{l^{\prime}}, ..\right)$. Here we do not focus on the form of the function $f$, but use that it is independent of $y_{R+1}$. After each transformation, the residuary noise becomes: $N_{k}^{r e s}=N_{m}+(1-\rho)^{2} N_{k-1}^{r e s}$. The recursion gives the total residuary noise as

$$
N_{R+1}^{r e s}=N_{R+1}+(1-\rho)^{2} N_{R}^{r e s}=N_{R+1}+(1-\rho)^{2} N_{R}+(1-\rho)^{4} N_{R}+\ldots+N_{1}(1-\rho)^{2 R}
$$

Using that for all $m: N_{m}=N_{m-1}=N /(R+1)$ :

$$
N_{R+1}^{r e s}=N_{m} \sum_{m=1}^{R+1}(1-a b)^{2 m}=\frac{N}{R+1} \frac{1-(1-\rho)^{2(R+1)}}{1-(1-\rho)^{2}}
$$

One can use the same approach as in section 4.4.1 and represent $M_{m l}=\sum_{l^{\prime}, l^{\prime \prime} . .} W_{k l} W_{l l^{\prime}}$. . as $\mathbf{M}=\mathbf{W}^{R}$. In the high SNR limit, we can consider the problem in the case of the nearest neighbors, so that the distortion leads to the error only between the nearest neighbors, and, therefore, in the transfer matrix $\mathbf{M}$ (see Eq. 4.20) only the non-zero elements are diagonal $\widetilde{m}$ and neighboring $\bar{m}=(1-\widetilde{m}) / 2$. Hence, the formula can be written as:

$$
\begin{gathered}
p\left(y_{R+1} \mid x_{k}\right)=\frac{\widetilde{m}}{\sqrt{2 \pi N_{R+1}^{\text {res }}}} \exp \left(-\frac{\left(y_{R+1}-\tilde{x}\right)^{2}}{2 N_{R+1}^{\text {res }}}\right) \\
\left.+\frac{\bar{m}}{\sqrt{2 \pi N_{R+1}^{\text {res }}}} \exp \left(-\frac{\left(y_{R}-x^{+}\right)^{2}}{2 N_{R+1}^{\text {res }}}\right)+\frac{\bar{m}}{\sqrt{2 \pi N_{R+1}^{\text {res }}}} \exp \left(-\frac{\left(y_{R}-x^{-}\right)^{2}}{2 N_{R+1}^{\text {res }}}\right)\right]
\end{gathered}
$$

where notations with tilde for the diagonal elements and \pm for neighboring points. Here $\tilde{x}$ and $x^{ \pm}$are real values resulting from integration and may be calculated from $f\left(x_{k}, x_{l}, x_{l^{\prime}}, ..\right)$. However, here we can avoid these calculations as we do not need the exact values of $\tilde{x}$ and $x^{ \pm}$, so we use different notations to distinguish between them.

We continue the same approach as in section 4.4.1 and calculate conditional entropy as:

$$
H_{y \mid x}=-\sum_{k=1}^{K} P_{k} \int_{-\infty}^{\infty} d y_{R+1} p\left(y_{R+1} \mid x_{k}\right) \log _{2} p\left(y_{R+1} \mid x_{k}\right)
$$




\subsection{Capacity calculation for regenerative mapping}

here $K$ is the constellation size. Substituting conditional pdf in a form of Eq. 4.55:

$$
\begin{aligned}
H_{y \mid x}=- & \sum_{k=1}^{K} \frac{P_{k}}{\sqrt{2 \pi N_{R+1}^{\text {res }}}} \int_{-\infty}^{\infty} d y_{R+1}\left(\tilde{m} \exp \left(-\frac{\left(y_{R+1}-\tilde{x}\right)^{2}}{2 N_{R+1}^{\text {res }}}\right)+\bar{m} \exp \left(-\frac{\left(y_{R}-x^{+}\right)^{2}}{2 N_{R+1}^{\text {res }}}\right)+\right. \\
& \left.\bar{m} \exp \left(-\frac{\left(y_{R}-x^{-}\right)^{2}}{2 N_{R+1}^{\text {res }}}\right)\right) \log _{2}\left[\frac{\tilde{m}}{\sqrt{2 \pi N_{R+1}^{\text {res }}}} \exp \left(-\frac{\left(y_{R+1}-\tilde{x}\right)^{2}}{2 N_{R+1}^{\text {res }}}\right)+\right. \\
& \left.\frac{\bar{m}}{\sqrt{2 \pi N_{R+1}^{\text {res }}}} \exp \left(-\frac{\left(y_{R}-x^{+}\right)^{2}}{2 N_{R+1}^{\text {res }}}\right)+\frac{\bar{m}}{\sqrt{2 \pi N_{R+1}^{\text {res }}}} \exp \left(-\frac{\left(y_{R}-x^{-}\right)^{2}}{2 N_{R+1}^{\text {res }}}\right)\right]
\end{aligned}
$$

Let us consider the first term

$$
\begin{gathered}
\tilde{\mathrm{I}}=\int_{-\infty}^{\infty} d y_{R+1} \frac{\tilde{m}}{\sqrt{2 \pi N_{R+1}^{\text {res }}}} \exp \left(-\frac{\left(y_{R+1}-\tilde{x}\right)^{2}}{2 N_{R+1}^{\text {res }}}\right) \log _{2}\left[\frac{\widetilde{m}}{\sqrt{2 \pi N_{R+1}^{\text {res }}}} \exp \left(-\frac{\left(y_{R+1}-\tilde{x}\right)^{2}}{2 N_{R+1}^{\text {res }}}\right)\right. \\
\left.+\frac{\bar{m}}{\sqrt{2 \pi N_{R+1}^{\text {res }}}} \exp \left(-\frac{\left(y_{R}-x^{+}\right)^{2}}{2 N_{R+1}^{\text {res }}}\right)+\frac{\bar{m}}{\sqrt{2 \pi N_{R+1}^{\text {res }}}} \exp \left(-\frac{\left(y_{R}-x^{-}\right)^{2}}{2 N_{R+1}^{\text {res }}}\right)\right]
\end{gathered}
$$

in the limit of large SNR and/or large number of nonlinear regenerators, we can leave under the logarithm only the first term:

$$
\begin{gathered}
\tilde{\mathrm{I}} \simeq \int_{-\infty}^{\infty} d y_{R+1} \frac{\widetilde{m}}{\sqrt{2 \pi N_{R+1}^{\text {res }}}} \exp \left(-\frac{\left(y_{R+1}-\tilde{x}\right)^{2}}{2 N_{R+1}^{\text {res }}}\right) \log _{2}\left[\frac{\widetilde{m}}{\sqrt{2 \pi N_{R+1}^{\text {res }}}} \exp \left(-\frac{\left(y_{R+1}-\tilde{x}\right)^{2}}{2 N_{R+1}^{\text {res }}}\right)\right] \\
=\int_{-\infty}^{\infty} d y_{R+1} \frac{\widetilde{m}}{\sqrt{2 \pi N_{R+1}^{\text {res }}}} \exp \left(-\frac{\left(y_{R+1}-\tilde{x}\right)^{2}}{2 N_{R+1}^{\text {res }}}\right) \log _{2}\left[\frac{1}{\sqrt{2 \pi N_{R+1}^{\text {res }}}} \exp \left(-\frac{\left(y_{R+1}-\tilde{x}\right)^{2}}{2 N_{R+1}^{\text {res }}}\right)\right] \\
+\widetilde{m} \log _{2} \widetilde{m}=-\frac{\widetilde{m}}{2} \log _{2}\left[2 \pi e N_{R+1}^{\text {res }}\right]+\widetilde{m} \log _{2} \widetilde{m}
\end{gathered}
$$

Similar for other terms, which after being summed (using $\left.\sum_{k=1}^{K} P_{k}=1\right)$ yield:

$$
H_{y \mid x} \simeq-\left(\widetilde{m} \log _{2} \widetilde{m}+2 \bar{m} \log _{2} \bar{m}\right)+\frac{\widetilde{m}+2 \bar{m}}{2} \log _{2}\left(2 \pi e N_{R+1}^{r e s}\right)
$$

Using that $\widetilde{m}+2 \bar{m}=1$ (due normalization of matrix $\mathbf{M}$ ) and the sum in the first brackets is given by Eq. 4.23 (further denoted as $H_{y \mid x}^{i d}$ ) with substitution $N / R \rightarrow N_{R+1}^{\text {res }}$, we obtain:

$$
\left.H_{y \mid x} \simeq H_{y \mid x}^{i d}\right|_{N / R \rightarrow N_{R+1}^{r e s}}+\frac{1}{2} \log _{2}\left(2 \pi e N_{R+1}^{r e s}\right)
$$

Similarly, we calculate output entropy as:

$$
H_{y}=-\int_{-\infty}^{\infty} d y_{R+1} p\left(y_{R+1}\right) \log _{2} p\left(y_{R+1}\right)
$$




\subsection{Capacity calculation for regenerative mapping}

where the output pdf is given by:

$$
p\left(y_{R+1}\right)=\sum_{k=1}^{K} P_{k} p\left(y_{R+1} \mid x_{k}\right)
$$

then substituting conditional pdf from Eq. 4.55 it can be simplified as:

$$
p\left(y_{R+1}\right)=\sum_{k=1}^{K} P_{k} \frac{1}{\sqrt{2 \pi N_{R+1}^{r e s}}} \exp \left(-\frac{\left(y-x_{k}\right)^{2}}{2 N_{R+1}^{\text {res }}}\right)
$$

Compare an analytical approximation Eq. 4.64 and a result of Eq. 4.63 with full numerical integration of the exact conditional pdf Eq. 4.44 plotted in Fig. 4.20. This, further substituted in Eq. 4.62 and integrated by using the method of steepest descent, results in:

$$
H_{y} \simeq-\sum_{k=1}^{K} P_{k} \log _{2}\left(P_{k}\right)+\frac{1}{2} \log _{2}\left[2 \pi e N_{R+1}^{r e s}\right]
$$
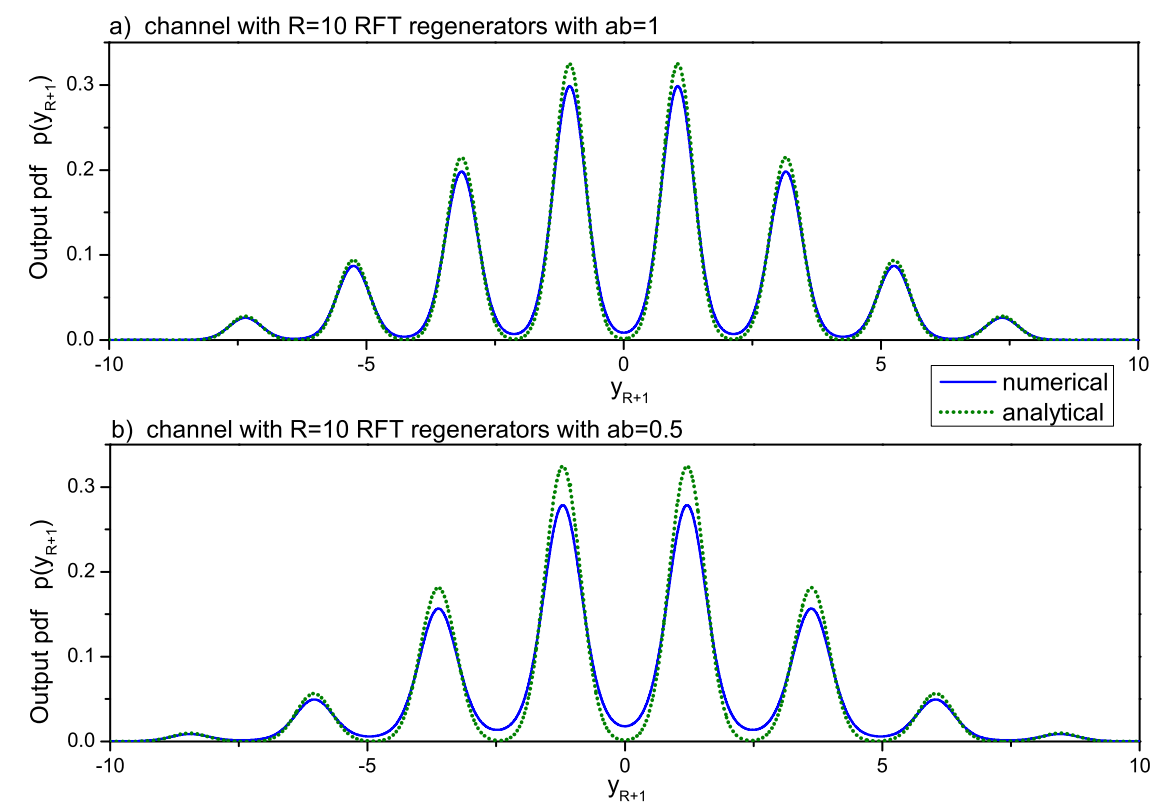

Figure 4.20: Output pdf - for $R=10$ and $S N R=10$ with $N=1$. Compare an analytical approximation Eq. 4.64 and the exact pdf calculated using Eq. 4.63 with full numerical integration of the conditional pdf Eq. 4.44 shown by dotted and solid lines correspondingly. 
The optimal input pmf: $P=P_{o p t}$ is found to be a discrete analogue of Gaussian distribution: $P_{k} \sim e^{-x_{k}^{2} / 2 S}$, see Fig. 4.15. Thus, we may apply the same reasoning as in Sec. 4.5 (see Eqs. 4.25-4.29) with substitution $N / R \rightarrow N_{R+1}^{\text {res }}$ to compute the sum, which is given by Eq. 4.29 and further denoted by $H_{y}^{i d}$. Therefore, the output entropy is equal to:

$$
\left.H_{y} \simeq H_{y}^{i d}\right|_{N / R \rightarrow N_{R+1}^{r e s}}+\frac{1}{2} \log _{2}\left[2 \pi e N_{R+1}^{r e s}\right]
$$

The capacity is defined as the difference of output and conditional entropies:

$$
C_{S}=\lim _{S N R \cdot R \rightarrow \infty} H_{y}-\left.H_{y \mid x}\right|_{P=P_{o p t}}=\frac{1}{2} \log _{2}(1+S N R)+\Delta C_{S}
$$

Using the method of steepest descent, we derive the capacity increase for the sine transfer function with the sub-optimal parameters relation (see Eq. 4.1) $\rho=a b \leq 1$ :

$$
\lim _{S N R \rightarrow \infty} \Delta C_{S}=\Delta C_{R}(R)-\log _{2}\left(\frac{1-(1-\rho)^{2(R+1)}}{1-(1-\rho)^{2}}\right)
$$

where $\Delta C_{R}(R)$ is given by Eq. 4.36. This result (Eq. 4.67) defines the upper capacity bound for the RFT mapping. Thus, in the limit of large SNR and/or large number of regenerators, all schemes tend to asymptotic behavior, when the gain gap between regenerative and linear AWGN channel capacity is constant. The saturation effect occurs when noise is squeezed to such a level that the stochastic distortion is small and the shift takes place within the plateau area. Thus, under such conditions, the system with the nonlinear regenerator is equivalent to the ideal regenerative system. In the limit of small noise, the conditional pdf approximates delta-function behavior and, consequently, reproduces the corresponding result of the channel with the ideal regenerators $\Delta C_{R}$. Thus, the capacity gain of any optimized regenerative system considered here tends to the asymptotic value defined further by Eq.4.36. This shows that the Shannon capacity with the optimized regeneration at high SNR saturates to the regenerative limit, with the Shannon capacity of the ideal regenerator $\left(\Delta C_{R}\right)$.

\subsection{Conclusion}

We have developed an analytical model that demonstrates the Shannon capacity of nonlinear regenerative channels higher than the linear limit - the Shannon capacity of 
the linear AWGN channel. The gain is achieved by the noise squeezing due to introduced nonlinear regenerative element. We presented the design rules for nonlinear regenerative systems. The introduced classes of nonlinear devices can be used for construction of communication channels with capacity exceeding the Shannon capacity of the linear AWGN channel. We anticipate that our results will lead to new insights into the Shannon capacity of nonlinear communications channels. 


\section{5 \\ Design and optimization of phase and amplitude regenerative channels}

\subsection{Introduction}

The exponentially increasing data traffic due to extensive use of broadband services imposes a continuing pressure for technological innovation in the physical layer of the optical network to meet the future demands on high data rate. With the optical transmission bandwidth being limited to $\sim 5 \mathrm{THz}$ by the commercially available erbium doped fiber amplifier technology, a dramatic increase in the spectral efficiency of the transmission link will be required (159). At the same time, the need to maintain a reducing cost per bit of transmitted data suggests that any serious attempt to upgrade system data rate should start from legacy network infrastructures before going to major deployments of new fiber cables, e.g. by adopting space division multiplexing technologies. The use of modulation formats with multi-level signaling represents a technologically mature and cost efficient way to achieve this goal (22). However, increasing the constellation complexity makes the signal more vulnerable to amplified spontaneous emission (ASE) noise and to a variety of linear and nonlinear fiber link impairments (23). To support transmission over long distances frequent all-optical regeneration can be used. Only regenerative channels can reduce the accumulation of amplified spontaneous emission noise and introduce contractive effects that can combat fiber nonlinear impairments. To minimize cost, in-line regeneration would ideally be compatible with complex constellations (160) and operate over multiple wavelengths 
simultaneously (161). Such features are most likely to be the result of all-optical approaches, making the use of advanced forward error correction (FEC) at a regeneration site difficult. Considering a fiber channel as the link between two nodes implementing FEC, all-optical regenerators would result in future high capacity fiber channels being highly nonlinear (72) even in the absence of nonlinear transmission impairments such as those considered in (2)-(21).

Thus, a new channel type arises, which we call a regenerative channel, where optimization of signal modulation and coding is crucial for high capacity transmission. Contrary to the well known linear cases or conventional nonlinear channels, where continuous bi-Gaussian constellations or ring constellations have respectively proved to be the optimum solution (23), for regenerative channels the problem still remains unsolved. Even for the conventional nonlinear channel, where nonlinearity plays only a detrimental role in signal transmission, significant benefits in achievable transmission rate can be acquired by optimizing the modulation format $(10,72,162)$. In regenerative channels, where nonlinearity has a higher and constructive impact, a stronger connection between the input modulation and the system parameters should be expected.

A number of regeneration techniques have been proposed making use of different nonlinear device technologies, e.g. semiconductor optical amplifiers (SOAs) (163)(166) or fibers $(41,61,66)$ in various subsystem configurations, e.g. Mach-Zehnder $(165,166)$ or non-linear loop mirrors (NOLMs) $(41,150,178)$. It has been shown $(41,150,163),(71)$ that most of them can support large regenerator cascades and bring significant improvements in the overall system transmission rate and transmission distance. However, they were primarily designed to address binary channels offering optimization only in the amplitude and pulse shape of the optical signal. Recent developments in all-optical signal processing have created a new type of nonlinear element, i.e. the phase sensitive amplifier (PSA) $(66,152)$ which can provide multi-level nonlinear response in the phase of the input optical field (66),(160). Due to this property PSA technology is compatible with a broader variety of signal constellations than binary ASK regenerators and can support the development of digital fiber links for long haul transmission at much higher spectral efficiencies. Recent experiments (71) have already demonstrated a cascadability of more than 100 PSA elements enabling transmission of BPSK signals over distances larger than $5000 \mathrm{~km}$. For higher spectral efficiencies a careful selection of the modulation format is required, due to the strong 
interplay between the noise and the non-linear transfer function of the PSA, to improve the overall transmission performance beyond the conventional limits of linear (1) and nonilnear optical channels (12), (72), (168) (see section 4).

\subsection{Phase regeneration}

Phase sensitive amplifiers have recently appeared as an ideal platform to support this functionality and subsystems for binary (63) and higher order phase encoded formats have already been demonstrated (66). Although these schemes have been designed and characterized for single stage demonstrations, their cascadability performance remains unknown. A rigorous optimization of their regenerative transfer function is necessary to achieve effective suppression of the transmission impairments in cascaded systems. A first approach has been proposed in (169), which is based on a "misfit factor" to minimize the difference between the nonlinear response of the PSA and the step-wise transfer function of an ideal multi-level quantizer. Here we developed an analytical methodology has been developed to optimize the transfer function of nonlinear regenerative systems based on the well-known theory of dynamic system analysis (170). This enables analytical predictions for optimal parameters to be made. Here, we apply the proposed analytical approach of to design PSA based regenerator cascades for multi-level phase encoded signal transmission. Our method is focused on the optimization of the transfer function that is responsible for the phase noise squeezing capabilities of the regenerator and affects its overall nonlinear response. In particular, through a set of simple mathematical conditions we define the optimal signal alphabet and identify the operating margins of the PSA cascade. The analytical predictions of our theory are verified by extensive numerical simulations of the transmission link performance. We show that in cascades the sensitivity to optimization increases and the derived analytical expression for the optimal PSA parameter ensures the best performance. The proposed model is generic and can be applied to any regenerative transfer function providing the possibility of dramatic improvements in the information capacity of future transmission systems (72).

A PSA element can be practically realized by a dual pump non-degenerate four wave mixing scheme of the input signal $r_{i n} e^{i \varphi_{i n}}$ with its $(M-1)-$ order harmonic (idler) (66). Assuming operation in the linear regime of the amplifier, i.e. the signal 
power is much lower than the power of the local pumps, the PSA transfer function is given by:

$$
r_{\text {out }} e^{i \varphi_{\text {out }}}=T\left(r_{\text {in }} e^{i \varphi_{\text {in }}}\right)=r_{\text {in }} e^{i \varphi_{\text {in }}}\left(1+m e^{-i M \varphi_{\text {in }}}\right)
$$

with the phase response:

$$
\varphi_{\text {out }}=F\left(\varphi_{\text {in }}\right)=\arctan \left(\frac{\sin \left[\varphi_{\text {in }}\right]+m \sin \left[(1-M) \varphi_{\text {in }}\right]}{\cos \left[\varphi_{\text {in }}\right]+m \cos \left[(1-M) \varphi_{\text {in }}\right]}\right)
$$

Note that the regenerative function depends only on the phase of the input signal. The parameter $m$ represents the amplitude ratio between the signal and its idler and has a critical role in defining the phase noise suppression properties of the PSA. Our recent analytical study (171) (see section 5.1.1) has identified $m=1 /(M-1)$ as the optimum value, which provides a flat phase response at the alphabet points of the signal, enabling maximum phase noise suppression and regenerative performance along the link. The amplitude and phase response of an 8-level PSA with an optimized $m$-parameter are depicted in Figs. 5.1(a)-5.1(b). Its phase response has a periodic staircase shape with plateau regions around the signal alphabet points. This periodicity suggests that only constellation diagrams with phase symmetry can be handled by the specific PSA scheme, although it can become the building block of more complex configurations that will allow dealing with rectangular M-QAM signals (68).

\subsubsection{Optimization of Cascaded Regenerative Links based on Phase Sensitive Amplifiers}

Here we develop an analytical method for optimizing phase sensitive amplifiers for regeneration in multilevel phase encoded transmission systems. The model accurately predicts the optimum transfer function characteristics and identifies operating tolerances for different signal constellations and transmission scenarios. The results demonstrate the scalability of the scheme and show the significance of having simultaneous optimization of the transfer function and the signal alphabet. The model is general and can be applied to any regenerative system.

In the above equations $m$ corresponds to the amplitude ratio of the interfering signal-idler pair of waves. It is an optimization parameter that defines the slope of 

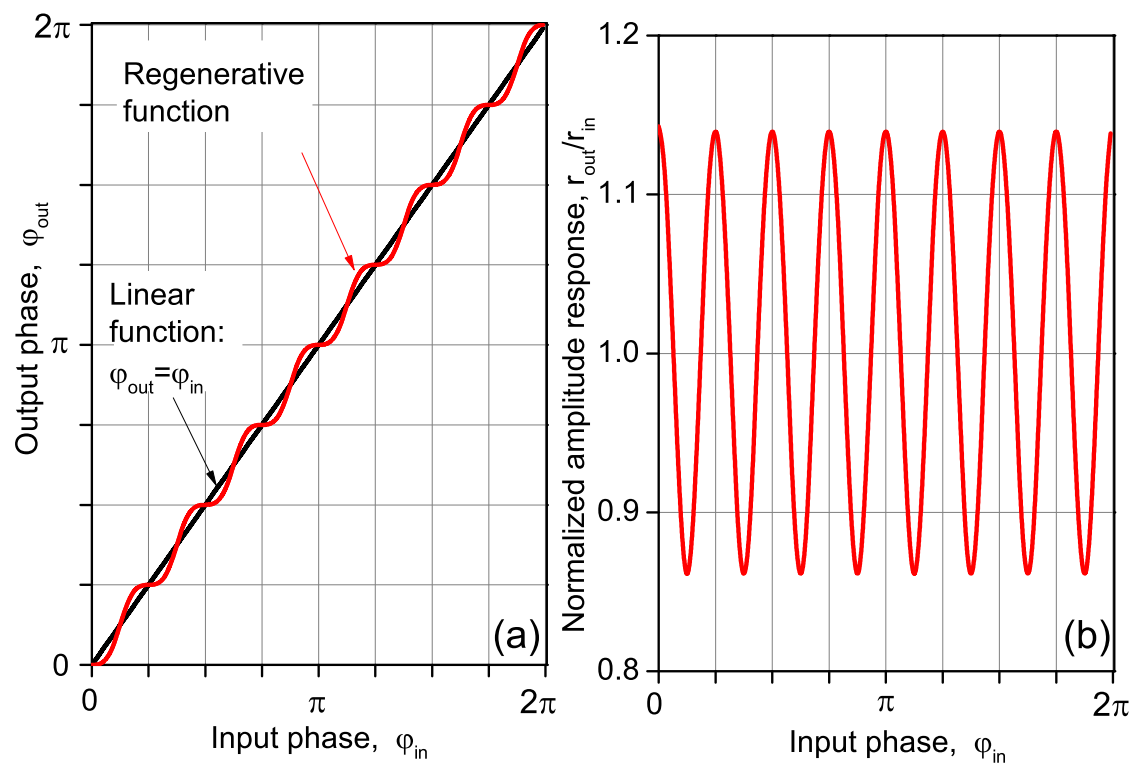

Figure 5.1: PSA response - (a) Normalized phase response and (b) normalized amplitude response of an 8-level phase sensitive amplifier as a function of the phase of the input signal.

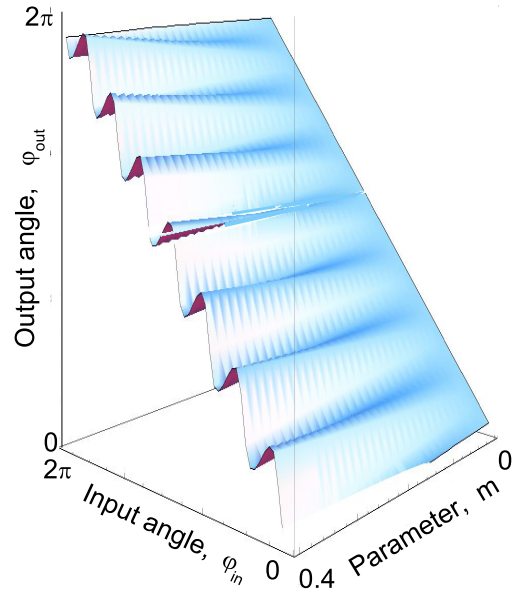

Figure 5.2: Periodicity and slope characteristics of the PSA phase transfer function

- with varying input phase $\varphi_{i n}$ and regenerative parameter $m$ with $M=8$ phase levels. 

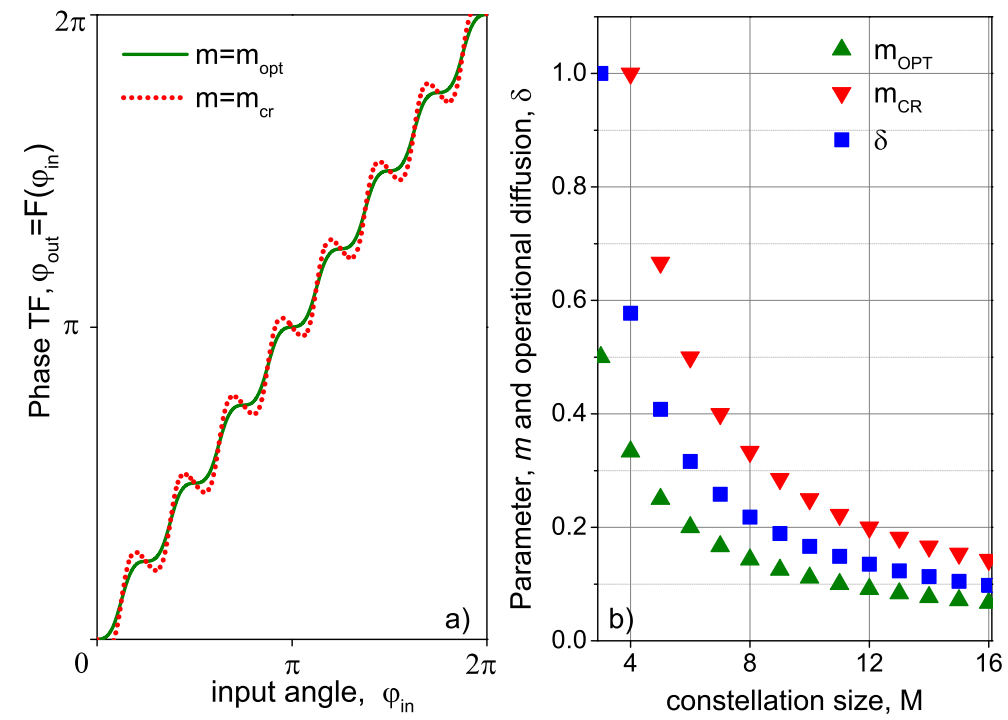

Figure 5.3: The PSA transfer function - a) PSA phase transfer function for 8-PSK format. The optimal value of the parameter $m_{\text {opt }}=1 / 7$ (shown by green solid line) demonstrates a plateau centered at the alphabet points, whereas a critical choice $m_{c r}=0.33$ (shown by red dashed line) degrades performance. b) Dependence of the optimal and critical values of $m$ and the attraction region's maximum half-width $\delta_{\max }$ on the order $M$ of the PSK modulation format. 

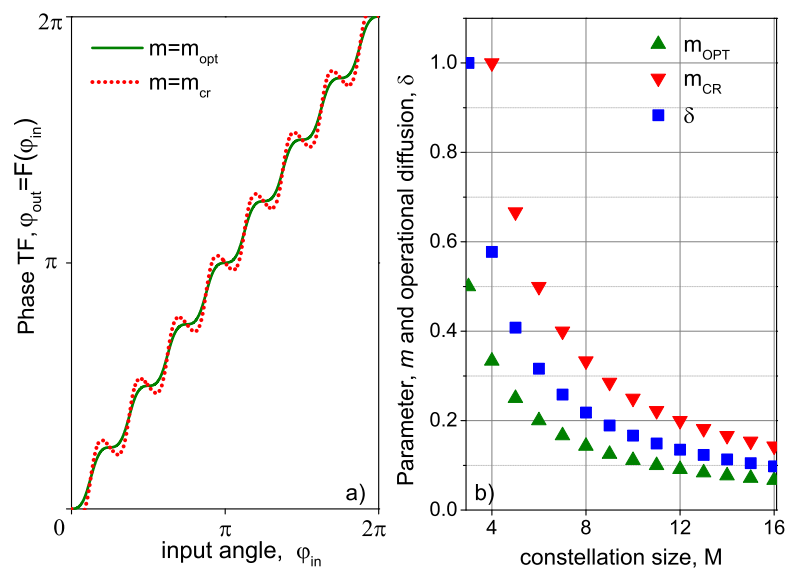

Figure 5.4: a) PSA phase transfer function for 8-PSK format. The optimal value of the parameter $m_{\text {opt }}=1 / 7$ (shown by green solid line) demonstrates a plateau centered at the alphabet points, whereas a critical choice $m_{c r}=0.33$ of the parameter (shown by red dashed line) is expected to lead to poorer regenerative performance. b) Dependence of the optimal and critical values of $m$ and the attraction region's maximum half-width $\Delta_{\max }$ on the order $M$ of the PSK modulation format.

the phase transfer function of the PSA near the alphabet points and characterizes its regenerative properties (see Fig. 5.2- 5.4). More specifically, the phase transfer function creates a periodic attractive potential around a set of special stationary points that are unaffected by the nonlinear transformation. Both, the stationary points and the attractive potential, contribute to the phase squeezing performance of the PSA. Clearly, the optimum set of values for parameter $m$ has to be identified to maximize the regeneration efficiency. Since the alphabet is discrete, it is also necessary to adapt the characteristics of the nonlinear element to the employed signal format and vice versa. Firstly, the nonlinear transfer function of the regenerator is adjusted to the signal alphabet. For this, the corresponding attraction regions should be centred at the corresponding alphabet points $\varphi^{*}$, the stationary points of the transformation, as defined by the conditions: $F^{\prime \prime}\left(\varphi^{*}\right)=0$ and $F\left(\varphi^{*}\right)=\varphi^{*}$. Thus, starting from Eq. 5.2, we derive the optimum constellation given by the set $\varphi^{*}=l \pi / M, l \in Z$. The next step is to ensure stability of the nonlinear transformation at the alphabet points for effective suppression of the phase noise. This condition leads to the inequality $\left|F^{\prime}\left(\varphi^{*}\right)\right|<1$, from which the 
variation limits of the regenerative parameter $m$ are derived as:

$$
|m|<m_{c r}, \quad m_{c r}=\frac{2}{M-2}
$$

here $m>0$ if $l=2 k$ and $m<0$ if $l=2 k+1$, with $k \in Z$. The points are superstable, i.e. $F^{\prime}\left(\varphi^{*}\right)=0$, when

$$
\left|m_{\text {opt }}\right|=\frac{1}{M-1}
$$

The superstable case creates operational plateaus around the alphabet points of the transfer function that enable maximum phase noise suppression. For $m \neq m_{\text {opt }}$ plateaus cannot be defined, however, provided that $|m|<m_{c r}$, partial suppression of the phase noise is still feasible within the limits of each attraction region, since $\left|F^{\prime}\left(\varphi^{*}\right)\right|<1$. An analytical expression can be derived for the attraction region's half-width $\delta$ in the limit of $\delta \ll 1$ by performing perturbation analysis on the equation $F^{\prime}\left(\varphi^{*}+\delta\right) \mid=1$, resulting in:

$$
\delta \simeq \sqrt{\left(1-\left|\frac{-1-m+m M}{1+m}\right|\right) /\left(\frac{m M^{3}(1-m)}{2(1+m)^{3}}\right)}
$$

here for simplicity we consider the absolute value $m=|m|$. The parameter $\delta$ has the meaning of the maximum phase noise distortion that can be suppressed by the regenerator. It acquires the maximal value $\delta_{\max }$ for the plateau condition $m=m_{o p t}$, whereas for sub-optimal $m$ values it narrows and tends to zero when $m$ approaches $m_{c r}$. Fig. 5.4a) depicts the phase transfer functions of regenerative PSAs with eight discrete phase states. The green solid line corresponds to the $m_{\text {opt }}$ selection of the regenerative parameter. The condition, $\left|F^{\prime}\left(\varphi^{*}\right)\right|<1$ results in simultaneous suppression of phase-to-phase and phase-to-amplitude noise conversion mechanisms. The red dashed line has been taken for the critical value $m_{c r}$, above which the PSA elements amplify phase noise and degrade the system performance. Fig. 5.4b) shows the dependence of the optimal and critical values of the regenerative parameter $m$ on the constellation size $M$. One can see that for high-order modulation formats the gap between $m_{o p t}$ and $m_{c r}$ is narrowed, making the PSA optimization more critical. The variation of the corresponding half-width $\delta_{\max }$ is also depicted in the same figure. 


\subsubsection{Simulation Model}

As in previous chapter we focused on the effect of noise squeezing due to nonlinear transformations. Thus, we have considered a transmission system of identical cascaded 3R regenerators (as in Fig. 4.1) based on PSA technology placed equidistantly along the fiber link, see Fig. 5.5a). Since the goal of this study is to capture the main performance features of the transmission system and to focus on the impact of the signal regenerative mechanisms in the selection of the optimum format, a simplified representation of the full system model has been adopted, where due to the properties of gating functionality of $3 \mathrm{R}$ element we can represent continuous-time signal $y(t)$ by its discrete-time form, see Fig. 5.5b). According to this, PSAs have been modelled by static elements of specific amplitude and phase response (66) and the impairments by additive white Gaussian noise (AWGN). AWGN can be considered as a representation not only of the amplified spontaneous emission noise $(108,172)$ (linear channel between regenerators), but also of the inter-channel distortions due to Kerr nonlinearity in the fiber (nonlinear channel between regenerators) (173), as well as, of the signalnonlinear noise interactions that might also arise (174). The validity of the approach has been verified both theoretically $(12,173)$ and experimentally $(175)$, and it has been widely used for estimating capacity limits in nonlinear fiber channels $(12,18,138)$.

Figure 5.5(b) illustrates the details of the proposed simplified simulation model. The propagation of the complex signal $Y_{k}$ at the $k^{t h}(k=1 \ldots R+1)$ regenerative section is described by the stochastic equation $Y_{k}=T\left(Y_{k-1}\right)+\eta_{k}$, where $T$ is the regenerative transfer function of the PSA and $\eta_{k}$ is the zero-mean AWGN noise of the $k$-th section with variance $N_{k}$, and $S$ is the average signal power at the input of the transmission link. To ensure that the average signal power is not changed by the regenerative transformation, we assume that the signal $T\left(Y_{k}\right)$ at the output of each PSA is normalized so that $\left\langle\left|Y_{k}\right|^{2}\right\rangle=\left\langle\left|T\left(Y_{k}\right)\right|^{2}\right\rangle$. We assume that within each section the fiber loss of each span is compensated by a subsequent amplifier and that deterministic impairments are ideally removed. We also note that our proposed model is generic and it can be used for various sets of parameters and amplification schemes. Whilst here we focused on the effectiveness of the noise suppression mechanisms rather than the origins of the Gaussian noise itself, effective channel signal to noise ratios may be readily calculated from physical link parameters using the conventional formalism. Thus for simplicity, 


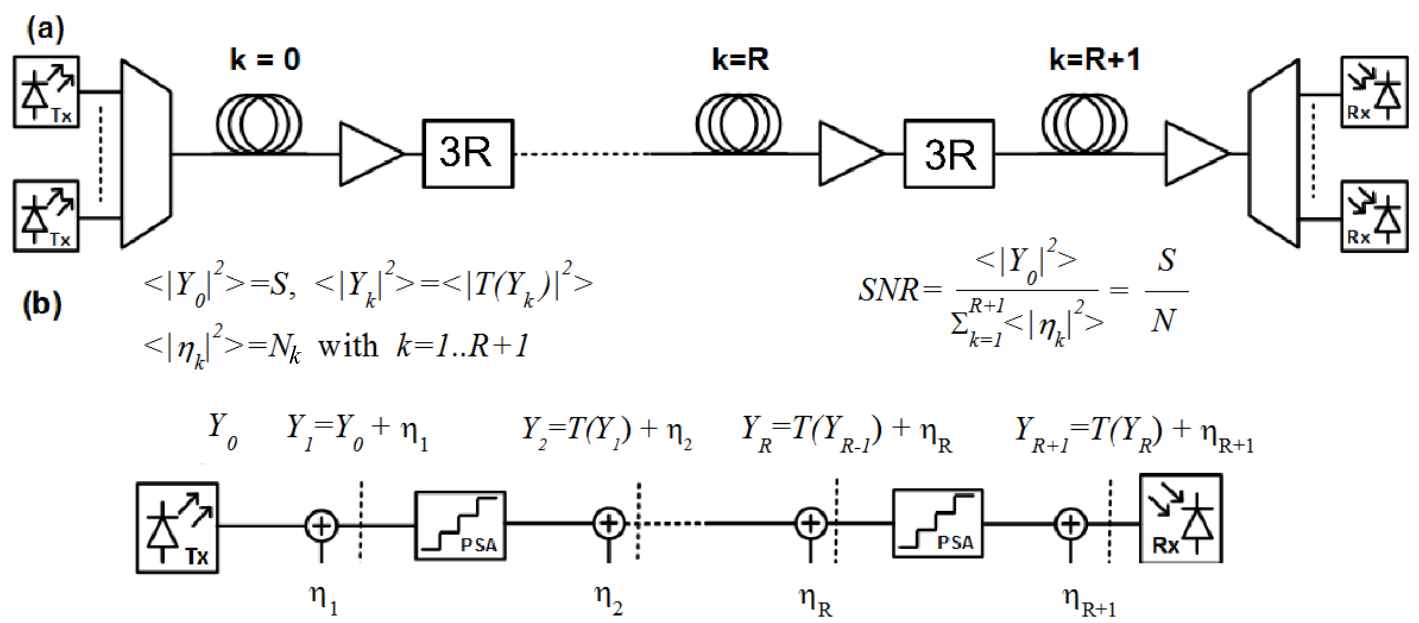

Figure 5.5: Schematic diagram - (a) of a future high capacity digital link formed by cascading $R$ PSA-based 3R-regenerators, $R+1$ links, each comprising a number of optically amplified spans adding noise with variance $N_{k}$, (b) Representation of simplified simulation model.

the point-to-point transmission system may be characterized by a single parameter, i.e. the signal-to-noise ratio (SNR), defined as the ratio of the average signal power at the input of the transmission system to the total power of the linearly added noise along the line:

$$
S N R=\frac{\left\langle\left|Y_{0}\right|^{2}\right\rangle}{\sum_{k=1}^{R+1}\left\langle\left|\eta_{k}\right|^{2}\right\rangle}=\frac{S}{\sum_{k=1}^{R+1} N_{k}}=\frac{S}{N}
$$

This is equivalent to the signal to noise ratio of the corresponding linear system having the same power of noise added to the signal during transmission, i.e. at the absence of regenerative elements, and provides a common reference for a fair comparison of systems with different regenerative properties.

In the considered regenerative system we have investigated numerically the transmission of different advanced modulation formats and have compared their performance by calculating the symbol error rate (SER) at the receiving end. The SER has been chosen as the most suitable figure of merit for performance characterization as it reflects directly the impact of regeneration on the transmitted symbols and enables comparison of different constellation diagrams, including those for which coding (including Gray coding) has not yet been developed. The SER has been calculated using 
the Monte Carlo method (176) on transmitted $2^{25}$-length sequences of randomly generated symbols.

\subsubsection{Optimization of transfer function parameter}

Next, the accuracy of the proposed analytical optimization method is verified by simulating numerically the performance of quasi-linear transmission regimes with cascaded PSA based regenerators. The PSAs have identical transfer functions and are placed equidistantly along the transmission line. The transmission impairments are represented by additive white Gaussian noise (AWGN) distributed uniformly along the line. The introduced degradation has been quantified in terms of signal to noise ratio (SNR) defined as the ratio of the input signal power to the power of the linearly accumulated noise along the transmission link, i.e. in the absence of PSA regenerators. This convention provides a common reference for the benchmarking of different nonlinear transmission channels (72). The system performance has been characterized in terms of symbol error rate (SER) calculated by direct error counting of $2^{23}$ Monte Carlo generated symbols. By comparing the SER while varying system parameters, we analyzed the general trends and evaluated the performance gain due to the regenerative functionality in the transmission system. Constellation diagrams of an 8-PSK signal after propagation through a transmission line of 10 cascaded PSAs at $S N R=12$ $\mathrm{dB}$ are depicted in Fig. 5.6. The results have been taken for different selections of the parameter $\mathrm{m}$, highlighting the influence of the PSA transfer function on the quality of the propagated signal. The un-regenerated case is depicted, in Fig. 5.6a). It is clear, that a significant SER improvement occurs for $m=m_{\text {opt }}$ (see Fig. 5.6b), when the PSA achieves efficient suppression of the induced phase noise. At the same time, a slight increase of the amplitude variation is noticed, as the gain of the PSA is also phase dependent. For $m \geq m_{c r}$ (see Fig. 5.6c) the phase distortion at each PSA stage is enhanced leading to a worse system SER performance than the un-regenerated case. However, the constellation shape of the signal maintains the basic star-like features of the regenerated case (middle panel) due to the sinusoidal amplitude response.

Figure 5.7 depicts the SER performance as a function of the SNR for various numbers of cascaded PSAs and for 4, 8, and 16-PSK modulation formats. For comparison the results are presented as two sets: the optimal and non-optimal values of the 
a) $\mathrm{m}=0$

$\log _{10}(S E R)=-1.5$

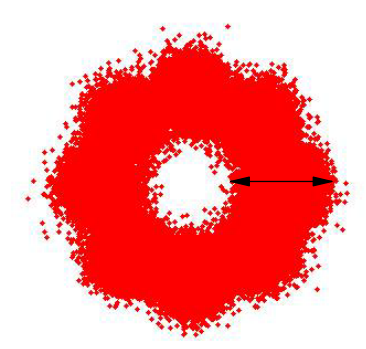

b) $m=1 / 7$

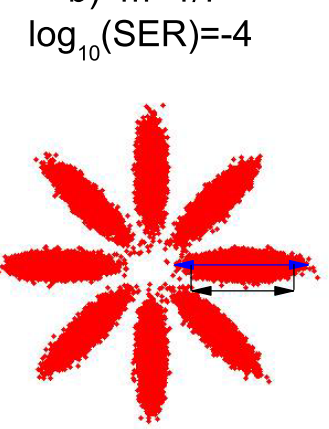

c) $\mathrm{m}=0.4$

$\log _{10}(\mathrm{SER})=-1$

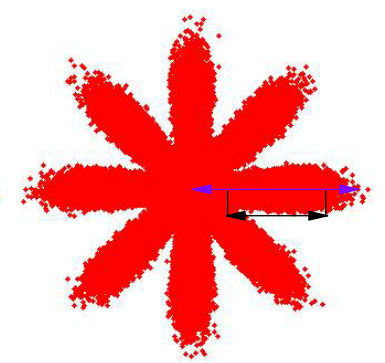

Figure 5.6: Constellation diagram of 8-PSK - after transmission with $S N R=12 \mathrm{~dB}$ a) without regeneration and after 10 PSAs in cascade with b)optimal $m=1 / 7$ and c) nonoptimal $m=0.4$. One can observe the dramatic gain in system performance due to the PSA optimization for the cascaded scenario. Arrows highlight the increase of amplitude noise (colored arrows) due to phase-to-amplitude noise conversion effect compared the amplitude noise in the absence of regeneration (black arrows). This effect is crucial in downgrading the SER for unoptimized systems (panel c).

m-parameter. For the non-optimal selection of $m$, a rapid degradation of the system performance occurs. On the contrary, when $m=m_{\text {opt }}$ the strongly regenerative behavior of the PSAs leads to a significant performance enhancement along the cascade, which is more profound for higher order modulation formats. Indeed, at SER of $10^{-3}$ the SNR improvement, with respect to the un-regenerated (linear) system, is only 1.5 $\mathrm{dB}$ for the QPSK signals, whereas for the 8 and 16- PSK signals it is $5.2 \mathrm{~dB}$ and 8 $\mathrm{dB}$, respectively. The results underline the increasing importance of all-optical regeneration with the constellation size of the transmitted signal. Specifically, they suggest that future PSA implementations should target modulation formats of higher order than QPSK, for larger improvements in system performance. As the number of cascaded PSAs increases, an asymptotic behavior in in performance improvement is also observed, see Fig. 5.7. This occurs because the transmission line is divided into a large number of sections and the noise power generated by each of them becomes significantly low to create phase errors. For higher order modulation formats it takes a larger number of inline regenerators to reach this saturation point as the signal sensitivity to phase noise is also increased. We have investigated further the performance dynamics 
along the PSA cascade by calculating the corresponding SNR improvement (for fixed $S E R=10^{-3}$ ) as a function of the regenerative parameter $m$.

Figure 5.8 depicts results for the case of an 8-PSK signal and for different numbers of inline regenerators. The aforementioned saturation effect in the performance is more apparent in this figure. By increasing the number of inline regenerators from $R=1$ to $R=2$, it gives the same SNR gain of $1 \mathrm{~dB}$, as going from $R=10$ to $R=20$. The dependence of the phenomenon on the regenerative parameter $\mathrm{m}$ is also shown. The saturation occurs faster for sub-optimal $m$ values. This is attributed to the non-ideal transfer function of the PSA, which allows an amount of residual phase distortion to accumulate between the regenerative sections enhancing the overall SER degradation. In Fig. 5.8, we have also identified the analytically predicted $m_{\text {opt }}$ values along with the corresponding optima $m_{\text {num }}$ indicated by the numerical simulations. A slight deviation is apparent between them for a small number of cascaded PSAs. However, since in this regime the system performance is relatively insensitive to $m$, the corresponding impact in SNR improvement is minimal, i.e. less than $4 \%$.

Figure 5.9 extends the results of the previous figure Fig. 5.8 to different modulation formats (i.e. 4, 8 and 16-PSK). We may observe that a plateau in the transfer function of the regenerator is not a necessary condition for demonstrating phase regeneration along the cascade. It is also possible to achieve effective noise suppression for suboptimal choices of the $m$-parameter. For example, selecting a suboptimal value $m=0.3$ gives, for the case of 8-PSK signals, an SNR improvement of $2 \mathrm{~dB}$, whereas the corresponding transfer function does not show any plateau-like behavior. On the other hand, non-optimal $m=0.36$ provides a similar transfer function, but it degrades the system performance compared to the channel in the absence of PSA, as it slightly exceeds the critical value of $m=0.33$, which our theory has predicted.

\subsubsection{Optimal packing for cascaded regenerative transmission based on phase sensitive amplifiers}

We investigate the transmission performance of advanced modulation formats in nonlinear regenerative channels based on cascaded phase sensitive amplifiers. We identify the impact of amplitude and phase noise dynamics along the transmission line and 

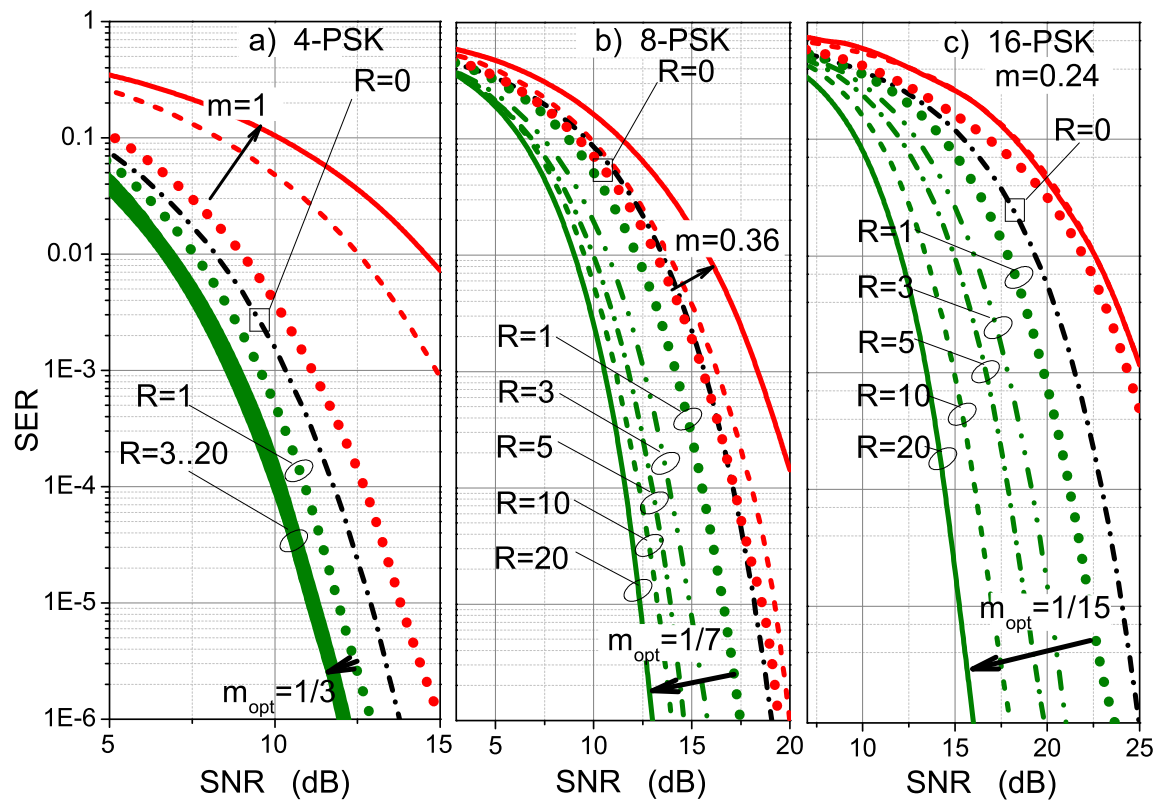

Figure 5.7: SER as a function of SNR with cascaded regenerative PSA elements - for a) 4-PSK, b) 8-PSK and c) 16-PSK formats. The SER of the linear channel is shown for comparison by black dash-dotted line. The SER of the system with the optimal and critical values of the parameter $m$ are shown by green and red curves, respectively. The arrows show the SER evolution for increasing number of in-line PSAs. 


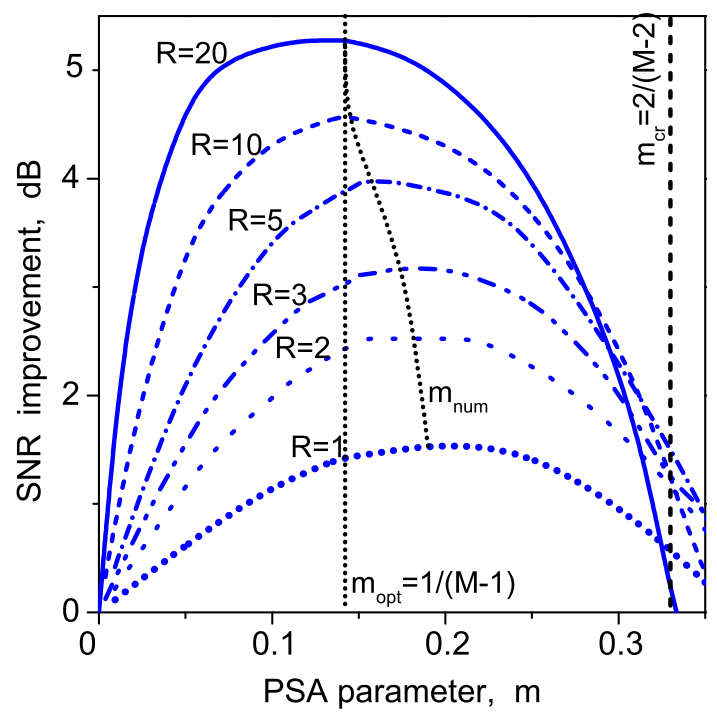

Figure 5.8: The dependence of the SNR improvement due to PSA application - for the fixed value $S E R=10^{-3}$ as a function of the PSA parameter $m$ for 8-PSK modulation format for the different number of PSAs (denoted by $R$ ). The convergence of the numerical values $m_{\text {num }}$ to the analytical $m_{\text {opt }}$ is shown by black dotted curve.

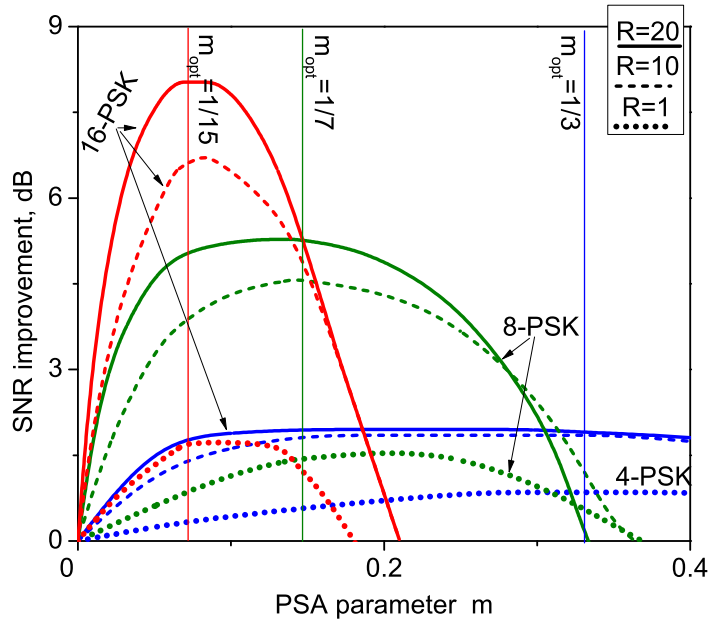

Figure 5.9: The dependence of the SNR improvement due to PSA application - for the fixed value $S E R=10^{-3}$ as a function of the PSA parameter $\mathrm{m}$ for 4 (blue), 8 (green) and 16 (red) PSK modulation formats. The cascaded scheme of 10 (dashed) and 20 (dotted) PSA elements gives significant improvement for high order PSK. The optimal value of $\mathrm{m}$ is shown by vertical lines of the corresponding color for each constellation size. 
show that after a cascade of regenerators, densely packed single ring PSK constellations outperform multi-ring constellations. This is due to phase-to-amplitude noise conversion, which significantly increases amplitude noise and, therefore, amplitude modulation is not effective. Thus, as the number of phase regenerators increases along the transmission link the optimum modulation format is single ring modulation - densely packed phase shift keying format. The results of this study will greatly simplify the design of future nonlinear regenerative channels for ultra-high capacity transmission.

\subsubsection{Results and discussion}

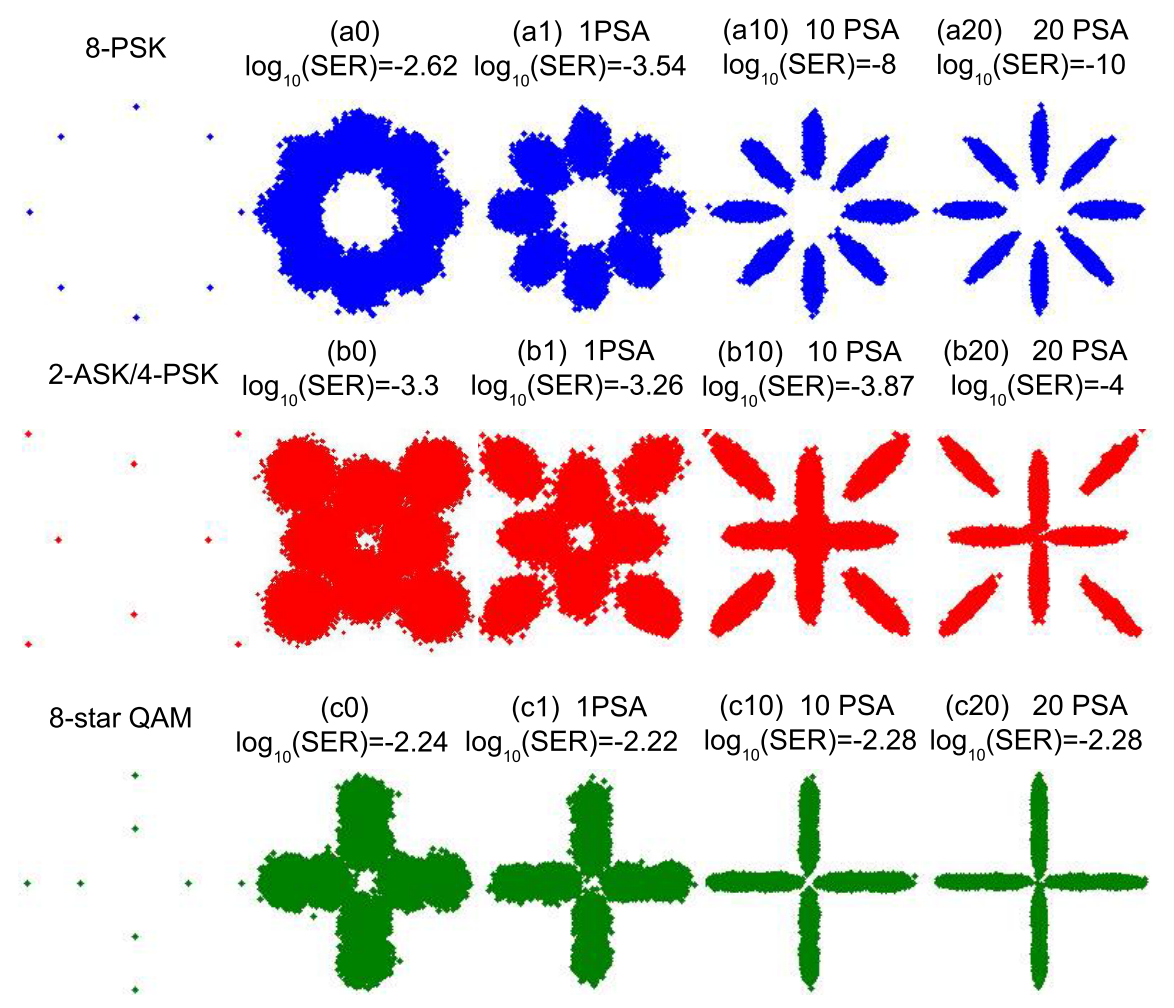

Figure 5.10: Phase symmetric constellations for 8-symbols - before (leftmost) and after transmission in linear channels and nonlinear regenerative channels with cascaded 1, 10, 20 phase regenerators for the fixed value of $\mathrm{SNR}=15 \mathrm{~dB}$. The M-level PSA was used with $M=8$ for a) and b) rows and $M=4$ for c) row. 


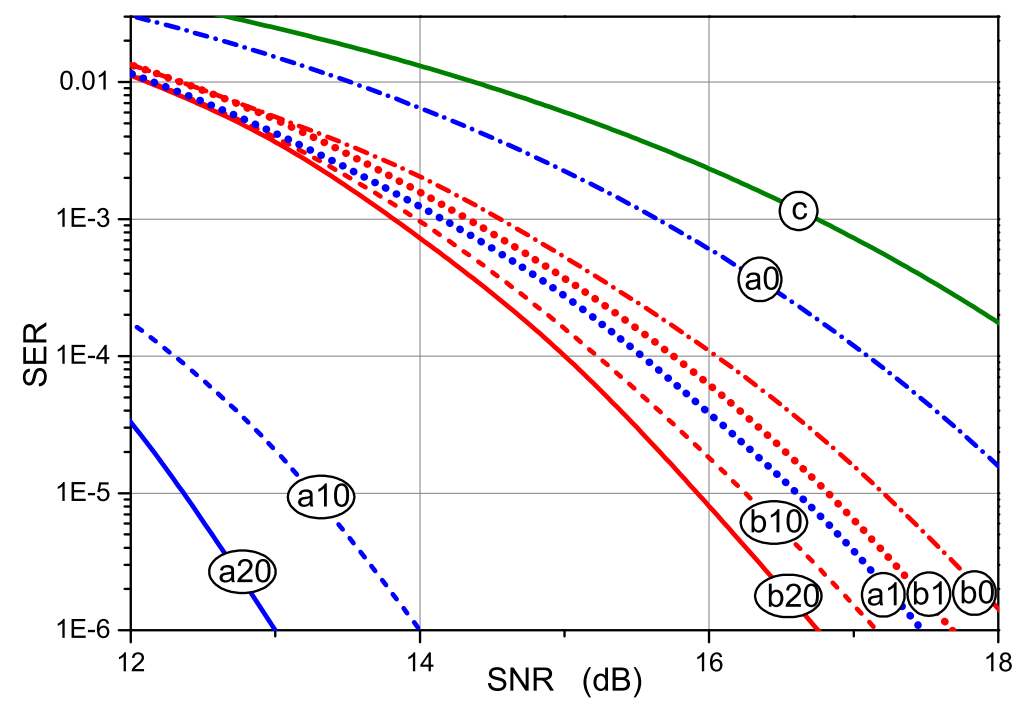

Figure 5.11: SERs as a function of SNR for the examined 8-symbol constellation types - 1 amplitude level: 8-PSK (index a, blue lines) and 2 amplitude levels: 2-ASK/4PSK (index b, red lines) and 8-star QAM (index c, green line) for linear channel (sub-index 0 ) and nonlinear regenerative channels with cascaded 1, 10, 20 phase regenerators (subindexes 1, 10, and 20 respectively). Formats are also identified by line color, whilst the number of regenerators in the link is identified by the dashing of the line (dash length increases with number of regenerators). 
Here we use simulation model described above and use optimal value of transformation parameter $m=1 /(M-1)$. We start our analysis by investigating the transmission performance of three different 8-symbol constellations with phase symmetry, as a function of the number of cascaded PSA regenerators in the link. The single ring 8PSK is compared against the 2-ASK/4-PSK and 8-star QAM formats, which combine phase shift keying and amplitude shift keying modulation. To make the comparison fair the average symbol energy was kept the same for all these cases. Figure 5.10 shows the corresponding constellation diagrams and symbol error rates (SER) calculated at fixed $\mathrm{SNR}=15 \mathrm{~dB}$ for the linear system (index 0 ), and when having 1,10 , and 20 regenerators in the link, indicated by respective indexes. The impact of PSAs in suppressing phase noise is apparent in each constellation and it becomes more effective when the number of in-line regenerative elements is increased. However, this does not necessarily bring improvement in the symbol error rate performance. From three examined cases, the PSK format experiences the largest improvement as its $\log _{10}(S E R)$ drops from 2.62 for the linear system to -10 after inserting 20 equally spaced PSA regenerative elements into the same system. For the 2-ASK/4-PSK the SER improves significantly less in comparison to the non-regenerated case, whilst for the 8-star QAM the improvement is negligible for regenerators with either $M=4$ or $M=8(M=4$ is shown in Fig. 5.10). This is because the latter two formats are more susceptible to amplitude distortions, which remain unsuppressed along the transmission line and, moreover, their performance is further degraded by the phase-to-amplitude conversion mechanism in the PSA, see Fig. 5.1(b).

The SER performance of the 8-star QAM cannot be improved due to the dominance of unsuppressed amplitude noise see curves c10 and c20 in Fig. 5.10. Therefore, their use is not suitable for PSA based regenerative links as it brings negligible impact in performance improvement. Furthermore, despite the $\pi / 4$ phase shift the points of the 2-ASK/4-ASK constellation have experienced, the robustness of the format against amplitude distortion has not been increased due to the erroneous transitions between the inner ring points (see constellations with index b in Fig. 5.10), that are still high and affect the overall SER performance.

The SER as a function of the SNR for the aforementioned 8-symbol constellations and for different number of regenerative elements is shown in Fig. 5.11. As expected, in the absence of in-line regeneration the best performing format is the 2-ASK/4-ASK, 
followed by the 8-PSK and then the 8-star QAM. Placing a phase regenerator in the middle of the link changes this order as the 8-PSK is improved significantly in contrast to the other two cases. For the latter formats a slight SER degradation is noticed, due to phase-to-amplitude noise conversion. The influence of this mechanism can be moderated by cascading more PSA elements in the link to bring down the average phase noise power so that a minimum amount of additional amplitude noise is created.
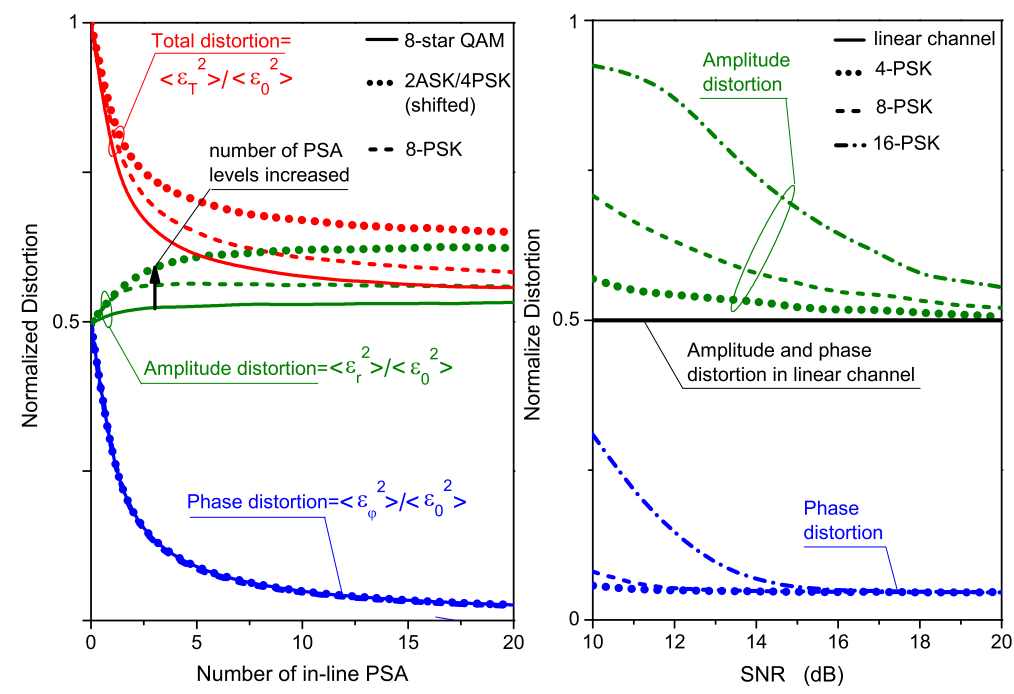

Figure 5.12: Amplitude and phase error for the received signal. - (a) Phase, amplitude and total distortion of the output constellation (b) as a function of the number of in-line regenerators for the fixed value $\mathrm{SNR}=15 \mathrm{~dB}$ and (c) as a function of the SNR for 10 cascaded regenerators.

We have analyzed further the amplitude and phase distortion dynamics of the aforementioned constellations at the receiver as a function of the number of cascaded regenerators in the transmission line. Figure 5.12(a) shows how the errors in amplitude $\varepsilon_{r}=\left\langle\left(r_{\text {out }}-r_{\text {in }}\right)^{2}\right\rangle$ and phase $\varepsilon_{\varphi}=\left\langle r_{\text {in }}^{2}\left(\varphi_{\text {out }}-\varphi_{\text {in }}\right)^{2}\right\rangle$ were defined with respect to the individual error vectors $\varepsilon_{T}=\left\langle\left|r_{\text {out }} e^{i \varphi_{\text {out }}}-r_{\text {in }} e^{i \varphi_{\text {in }}}\right|^{2}\right\rangle$. Figures 5.12(b,c) depict the total, amplitude and phase distortions, normalized to the total accumulated noise power of the linear system $\left\langle\left|\varepsilon_{0}\right|^{2}\right\rangle$. The normalization factor is independent on the type of modulation format and is a function of SNR only. Therefore, it allows us to make fair comparison of the distortion in the units of the linear system (in the linear system the 


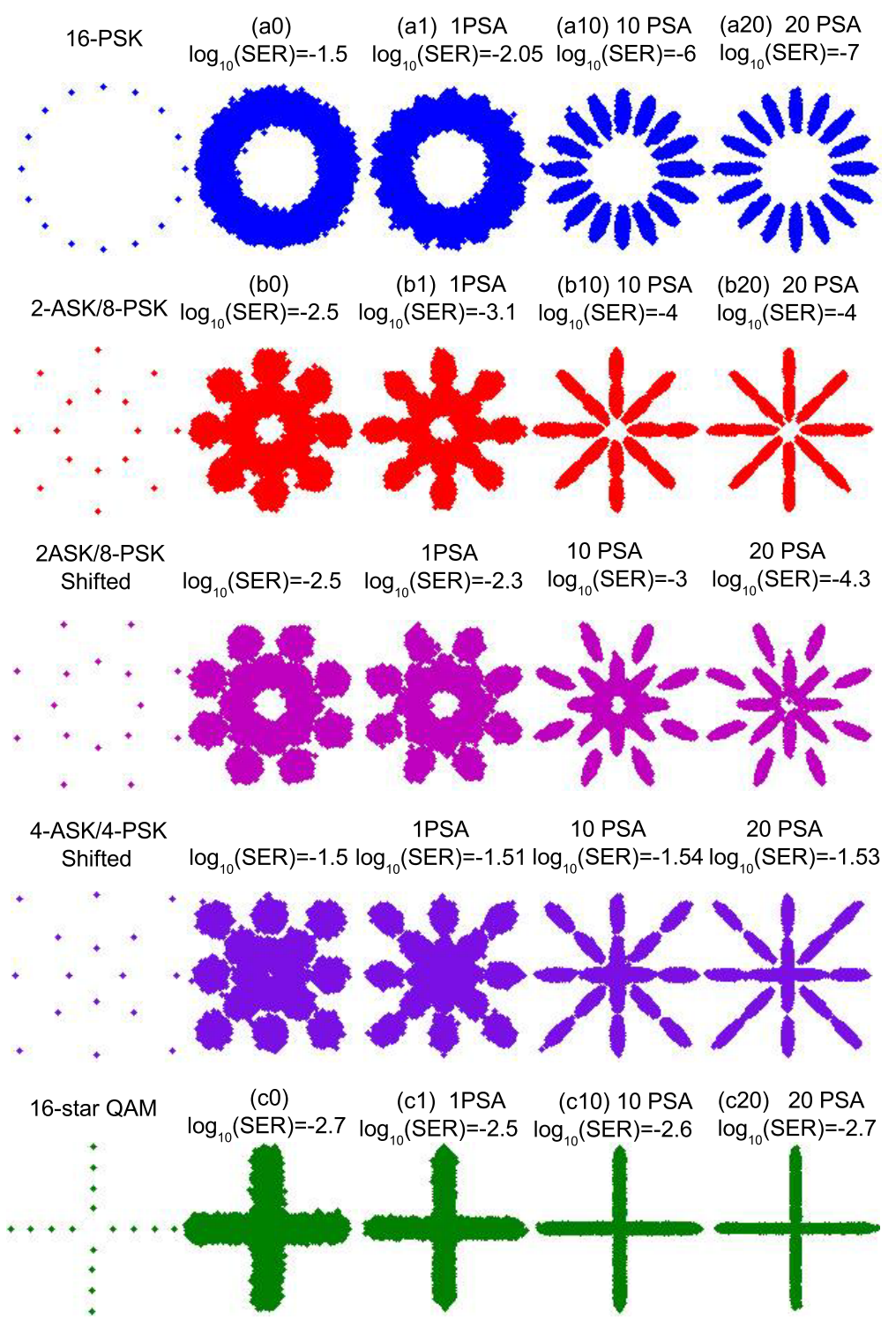

Figure 5.13: Phase symmetric constellations formats for 16 symbols - before (leftmost) and after transmission in linear channels (index 0 ) and nonlinear regenerative channel with cascaded 1, 10, 20 phase regenerators (indexes 1,10, and 20 respectively) for the fixed value $\mathrm{SNR}=18 \mathrm{~dB}$. The M-level PSA was used with $M=16$ for a) and c) rows, $M=8$ for b) and $M=4$ for d) row. 


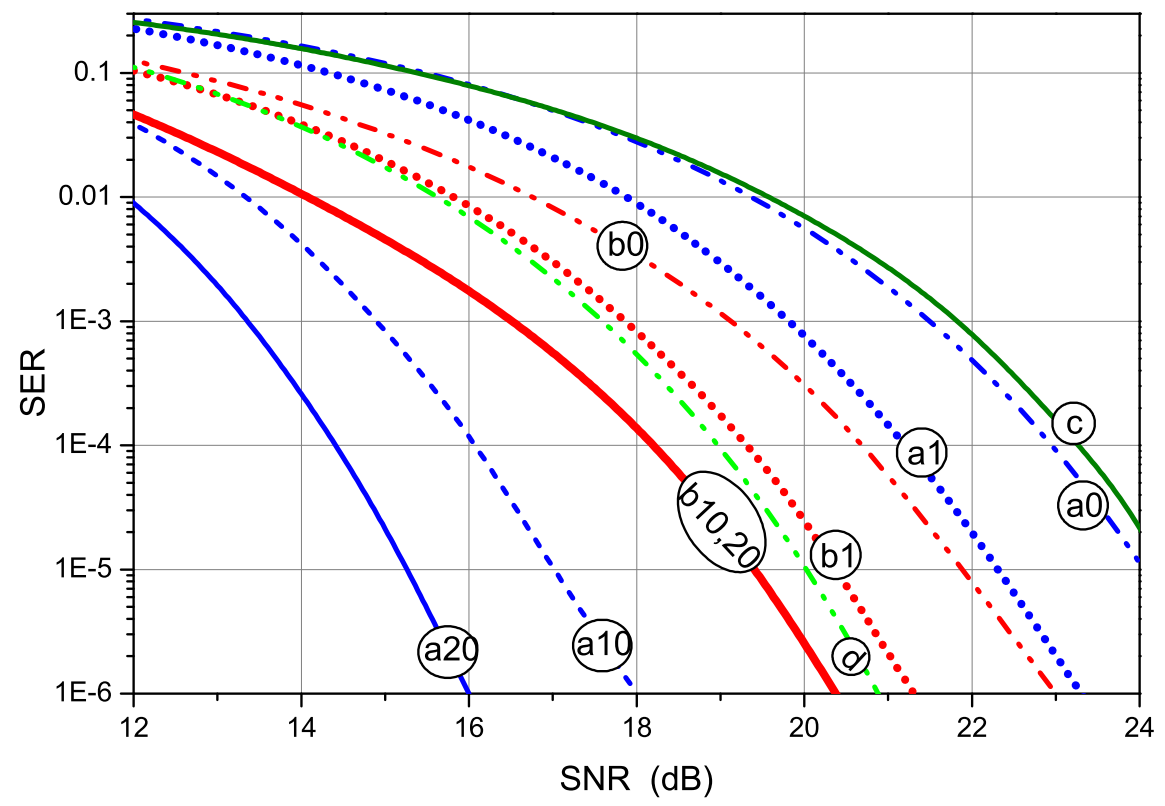

Figure 5.14: The SERs as a function of SNR for the various types of modulation formats - shown in Fig. 5.13, 1 amplitude level: 16-PSK (index a), 2 amplitude levels: 2ASK/8PSK (index b), and 4 amplitude levels: 16-star QAM (index c) and 16-QAM (index d) for linear channel (index 0) and nonlinear regenerative channels with cascaded 1, 10, 20 phase regenerators (indexes 1, 10 and 20 respectively). Formats are also identified by line color, whilst the number of regenerators in the link is identified by the dashing of the line (dash length increases with number of regenerators). 
normalized total distortion is unity and phase and amplitude distortions are equal to 0.5). In Fig. 5.12(b) the transmission link was characterized by a fixed SNR of $15 \mathrm{~dB}$. Note, that for large SNR values, when the phase noise is small so that the phase distortion occurrs within the plateau area, increasing the number of in-line regenerators reduces the accumulated phase distortion at the end of the link equally for each of the examined formats. However, for lower SNRs values, signals with a lower number of phase states are expected to have good performance as they will experience a more effective suppression of their phase noise from the corresponding PSA transfer function. This is demonstrated in Fig. 5.12(c), where the normalized distortion (in green the amplitude distortion and in blue the phase distortion) has been calculated for signals of different PSK order as a function of the SNR at the end of a line with 10 cascaded regenerators. The influence of the PSA transfer function on the accumulation of the amplitude noise is also depicted in Figs. 5.12(b,c). Due to the PSA induced phase-toamplitude conversion an increase of the amplitude distortion is observed at the end of the line, which is different for each constellation. Specifically, 8-star QAM signals experience the least amplitude noise enhancement, as they are processed by 4-level PSAs that are more robust to this effect, compared to the other two cases (2-ASK/4-ASK, 8PSK), which require PSAs of 8-levels. Furthermore, since the nonlinear transformation induces a mixing of signal and noise, the phase-to-amplitude noise is additionally enhanced for two amplitude level signals compared to the PSK formats with the same average energy, see Fig. 5.12(c). However, the enhancement is incremental and saturates to a constant value as the number of regenerators is increased.

Finally, we have extended the analysis to modulation formats of higher complexity by considering 16-symbol constellations of different geometry and evaluating their performance in systems of cascaded phase regenerators. The performance of the single ring 16-PSK constellation has been benchmarked against the dual ring diagrams of 2-ASK/8-PSK and 2-ASK/8-PSK shifted, the four-ring 4-ASK/4-PSK shifted format, and finally the 16-star QAM. Figure 5.13 depicts the corresponding constellation diagrams at the input of the link as well as at the receiver after different numbers of cascaded regenerators. For the formats presented here, the effects of phase regeneration discussed above become more pronounced, in particular the trade-off between the efficiency of phase squeezing and the impact of phase-to-amplitude conversion. 
The SER performance as a function of the SNR of the 16 point constellations of Fig. 5.13 is depicted in Fig. 5.14. Though amplitude modulated formats surpass densely-packed PSK for linear transmission, as the number of PSAs increases the SER associated with 16-PSK is gradually improved, and eventually the 16-PSK outperforms all other examined formats. Therefore, PSK signal modulation proves to be the most beneficial for transmission in PSA based regenerative links as it experiences the best SER improvement. As with the 8-symbol example, this is attributed to the intrinsic robustness of the PSK format against the amplitude noise, whereas, the multi-ring constellations are more vulnerable to this effect. Applying phase shift on the different rings of the constellations does not bring major improvement in their SER performance.

Since our goal was not to confine the study to a particular transmission scenario assuming a fixed distance between consecutive regenerators, on the contrary, we preferred to build a rather generic model of the transmission system with in-line PSAs, which can be used for various sets of parameters and amplification schemes. The length of the transmission line can be directly related to the effective SNR of the equivalent linear system at the receiving end. In particular, assuming operation with EDFAs, the SNR formula (see Eq. 5.6) may be transformed as follows:

$$
S N R=\frac{\left\langle\left|Y_{0}\right|^{2}\right\rangle}{\sum_{k=1}^{R+1}<\left|\eta_{k}\right|^{2}>}=\frac{\left\langle 2\left|Y_{0}\right|^{2}\right\rangle}{N_{A}\left(e^{\alpha L_{a}}-1\right) N F h v_{s} B}
$$

where $\left\langle\left|Y_{0}\right|^{2}\right\rangle$ is the average power of the input signal, $B$ is the signal bandwidth, $N_{A}$ is the number of amplified spans in the transmission line, $\alpha$ is the fiber loss per unit length and $L_{a}$ is the length of each amplified span. For a system of 16-PSK signals and system characterized by the following set of numerical parameters:

we calculate at the end of the line an $S N R=16.05 \mathrm{~dB}$. By making use of the proposed model we can then identify the number of required PSA regenerators in the link to bring down the SER to acceptable levels. In the specific example, the SER is improved from about $10^{-1}$ to $10^{-4}$, when 10 PSAs are equidistantly placed in the line at each $750 \mathrm{~km}$, or to $10^{-6}$ for the case of 20 cascaded PSA at each $375 \mathrm{~km}$. The SER results for this 16-PSK signal case were derived from Fig. 5.14 (for 8-symbol constellations see Fig. 5.11). 


\begin{tabular}{|l|l|}
\hline total length & $L=N_{A} L_{a}=100 \times 75 \mathrm{~km}=7500 \mathrm{~km}$ \\
\hline noise figure & $N F_{d B}=5 \mathrm{~dB}$ \\
\hline amplifier spacing & $L_{a}=75 \mathrm{~km}$ \\
\hline fiber loss per unit length & $\alpha=\frac{0.2}{10 \log _{10}(e)} \mathrm{km}^{-1}$ \\
\hline signal bandwidth & $B=33 \mathrm{GHz}$ \\
\hline average power of the input signal & $S=\left\langle\left|Y_{0}\right|^{2}\right\rangle=1 \mathrm{~mW}$ \\
\hline carrier frequency & $\mathrm{v}_{s}=193.41 \mathrm{THz}$ \\
\hline
\end{tabular}

Table 5.1: Transmission parameters.

\subsection{Design of multilevel amplitude regenerative system}

\subsubsection{Introduction}

A nonlinear optical loop mirror (NOLM) proposed in (177) was experimentally demonstrated as an efficient amplitude regenerator $(146,160,178)$. In particular, the cascadability of a NOLM in applications for long-haul transmission was experimentally proved $(41,61,69,70)$. However, the existing schemes are limited to 2 amplitude levels.

Here we propose and examine a novel scheme, which creates multiple regenerative levels in the amplitude transfer function. This is achieved by coupling the NOLM transformed signal with the original wave. The scheme is flexible: additional parameters give more freedom for optimization - the number of levels and their positioning can be easily varied. We demonstrate the model on various circular (from 16- to 256symbol) constellations and observe efficient amplitude regeneration. Moreover, the scheme is compatible with phase regenerators, which can be relevant for applications in coherent optical communications.

\subsubsection{Design}

The initial signal $A_{\text {in }}=\sqrt{P_{\text {in }}} e^{i \varphi_{\text {in }}}$ is split by a $3 \mathrm{~dB}$ coupler and then one of the waves is transformed by the NOLM. The corresponding transfer matrix of the coupler is given by:

$$
\widehat{\mathbf{C}}_{\mathbf{1}}=\frac{1}{\sqrt{2}}\left(\begin{array}{ll}
1 & \mathrm{i} \\
\mathrm{i} & 1
\end{array}\right)
$$




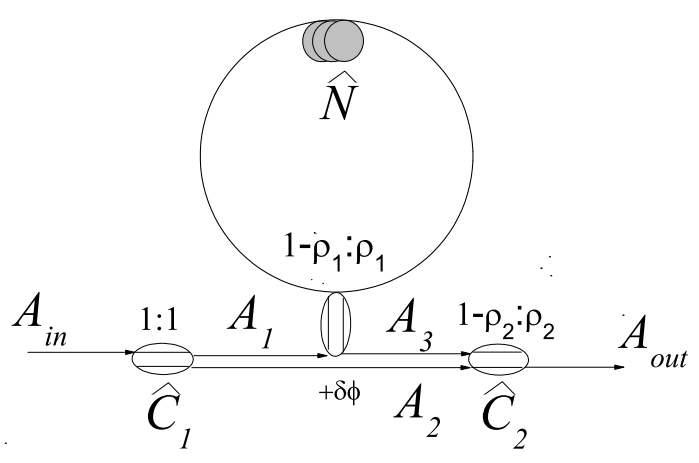

Figure 5.15: Scheme of the coupled NOLM - A NOLM, which induces nonlinear transformation given by due to coupling of counter-propagating waves with a coupling ratio $1-\rho_{1}: \rho_{1}$, is coupled with a linear path by two couplers with coupling ratio of the first coupler $1: 1$ for the first coupler (coupling matrix) and $1-\rho_{2}: \rho_{2}$ for the last coupler (coupling matrix). As a result, the amplitude response $P_{\text {out }}$ of the output wave $A_{\text {out }}$ versus input power $P_{\text {in }}$ of input wave $A_{\text {in }}$.

The NOLM transformation results in the following:

$$
\widehat{\mathbf{N}}\left(\sqrt{\frac{P_{\text {in }}}{2}}\right)=\left[-\left(1-\rho_{1}\right) e^{i\left(1-\rho_{1}\right) \gamma L P_{i n} / 2}+\rho_{1} e^{i \rho_{1} \gamma L P_{i n} / 2}\right] \sqrt{\frac{P_{\text {in }}}{2}}
$$

where $\rho_{1}$ denotes the coupling ratio, $L$ is the length of highly nonlinear fiber, and $\gamma$ is the fiber nonlinear coefficient. Then, the two waves (output of the NOLM and the second wave of the first coupler rotated by $\Delta \phi=3 \pi / 2$ ) are coupled by the second coupler with the power ratio between them being $\kappa^{2}$. The latter is a significant parameter, it can be adjusted as the characteristic of the second coupler: $\kappa=\sqrt{\rho_{2} /\left(1-\rho_{2}\right)}$ (see the scheme in Fig.5.15) where the transfer matrix is given by:

$$
\widehat{\mathbf{C}}_{2}=\left(\begin{array}{cc}
\sqrt{1-\rho_{2}} & \mathrm{i} \sqrt{\rho_{2}} \\
\mathrm{i} \sqrt{\rho_{2}} & \sqrt{1-\rho_{2}}
\end{array}\right)
$$

alternatively, having the second coupler to be $1: 1$ and placing an attenuator after the NOLM before the coupler. The obtained wave is amplified by $2 /\left(1-\rho_{2}\right)$ to restore the initial power. The resulting total transfer function is expressed via the initial field as:

$$
\begin{gathered}
A_{\text {out }}=\sqrt{P_{\text {out }}} e^{i \varphi_{\text {out }}}=\sqrt{P_{\text {out }}} e^{i\left(\varphi_{\text {in }}+\delta \phi\right)}= \\
\left(1+i \kappa\left[-\left(1-\rho_{1}\right) e^{i\left(1-\rho_{1}\right) \gamma L P_{\text {in }} / 2}+\rho_{1} e^{i \rho_{1} \gamma L P_{\text {in }} / 2}\right]\right) \sqrt{P_{\text {in }}} e^{i \varphi_{\text {in }}}
\end{gathered}
$$



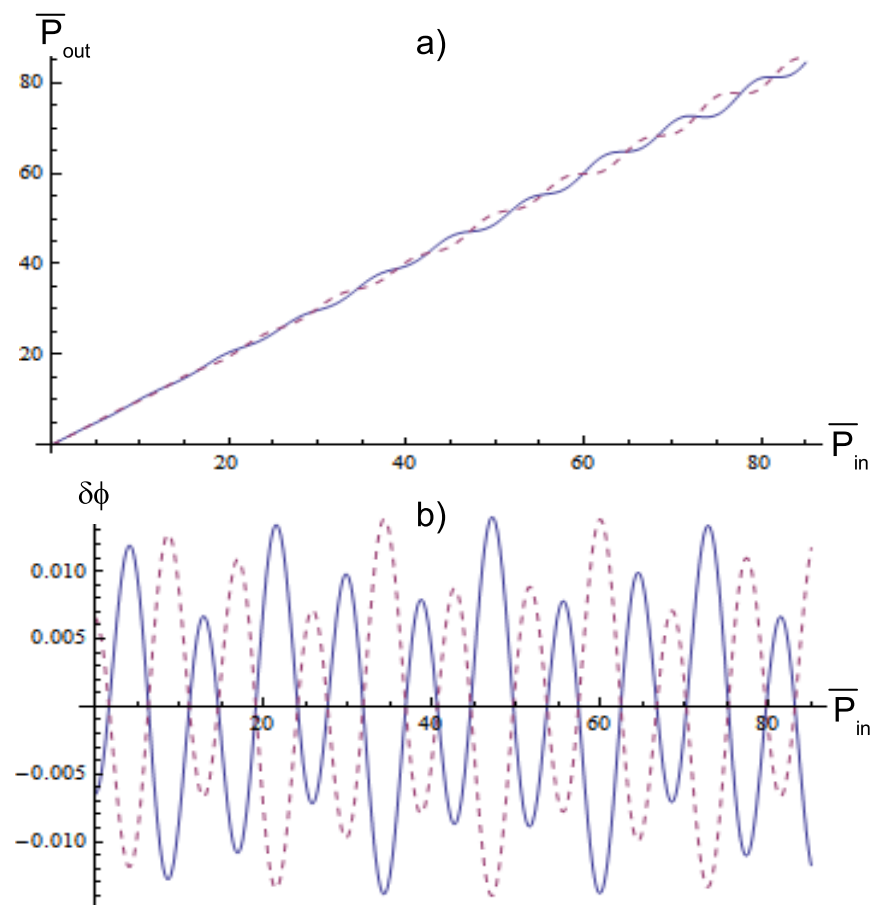

Figure 5.16: Transfer function of the coupled NOLM - (a) TF for the dimensionless output power normalized by $\gamma L / 2$ and (b) associated phase shift for parameters: $\rho_{1}=$ $0.267 ; \Delta \phi=3 \pi / 2 ; \kappa=\sqrt{\rho_{2} /\left(1-\rho_{2}\right)}=0.014$ (solid curves). By changing parameters as $\rho_{1}^{\prime}=1-\rho_{1}$ one can achieve sign-reversed phase shift and the amplitude levels will be shifted (dashed curves). 
As a result of the transformation, the transfer function (TF) of the output power versus the initial power has multiple plateau regions around the given stationary and stable points of the transformation (see Fig.5.16(a)). The number and positioning of the levels can be easily varied by adjusting TF parameters: $\rho_{1}$ and $\kappa$ by applying the optimization procedure of regenerative mapping $(168,171)$ (see section 4.3$)$ to the amplitude and phase response:

$$
\begin{array}{r}
P_{\text {out }}=P_{\text {in }}\left(1+2 \kappa^{2}-2 \kappa^{2}\left(1-\rho_{1}\right) \rho_{1} \cos \left[\left(1-2 \rho_{1}\right) \psi\right]+\right. \\
\left.2 \kappa\left[\left(1-\rho_{1}\right) \sin \left[\left(1-\rho_{1}\right) \psi\right]-\rho_{1} \sin \left[\rho_{1} \psi\right]\right]\right), \quad \psi=\frac{\gamma L P_{\text {in }}}{2} \\
\delta \phi=\arctan \left(\frac{\rho_{1} \kappa \cos \left(\rho_{1} \psi\right)-\left(1-\rho_{1}\right) \kappa \cos \left(\left(1-\rho_{1}\right) \psi\right)}{\left.1-\rho_{1} \kappa \sin \left(\rho_{1} \psi\right)+\left(1-\rho_{1}\right) \kappa \sin \left(1-\rho_{1}\right) \rho_{1} \psi\right)}\right)
\end{array}
$$

Also, the transformation is accompanied by an additional power-dependent phase shift (see Fig.5.16(b)), which is reduced compared with the traditional one NOLM scheme. Moreover, since the phase shift is sign-varied, by changing the parameters one can easily achieve phase shift to be sign-reversed. In particular, if one chooses $\rho_{1}^{\prime} \rightarrow$ $1-\rho_{1}$ or, alternatively, $\kappa^{\prime} \rightarrow-\kappa$ (or $\Delta \phi^{\prime} \rightarrow \Delta \phi+\pi$ ), then the new phase shift will be sign reversed (see Fig.5.16(b)) and the stable points of the amplitude TF will be shifted (see Fig.5.16(a)) to the different set of stationary points: $T\left(P_{i n}\right)=P_{i n}$. This highlights the importance of the regenerative mapping requirements (stability and stationarity) to be completely fulfilled for efficient regeneration.

The dimensionless output power $\bar{P}_{\text {out }}=\gamma L P_{\text {out }} / 2$ and phase shift $\delta \phi$ versus the normalized initial power $\bar{P}_{\text {in }}=\gamma L P_{\text {in }} / 2$ for parameters $\rho_{1}=0.267 ; \kappa=0.014$ are shown in Fig.5.16(a-b) correspondingly.

\subsubsection{Numerical simulations}

Next we demonstrate through numerical modelling the regenerative effect of the proposed scheme by simulating numerically the transmission of circular 16-, 64- and 256 - quadrature amplitude modulation (QAM) (also referred in literature as APSK) via a noisy channel. The amplitude regenerators are placed equidistantly in cascades along the line. Noise is modelled as an additive white Gaussian noise uniformly distributed 

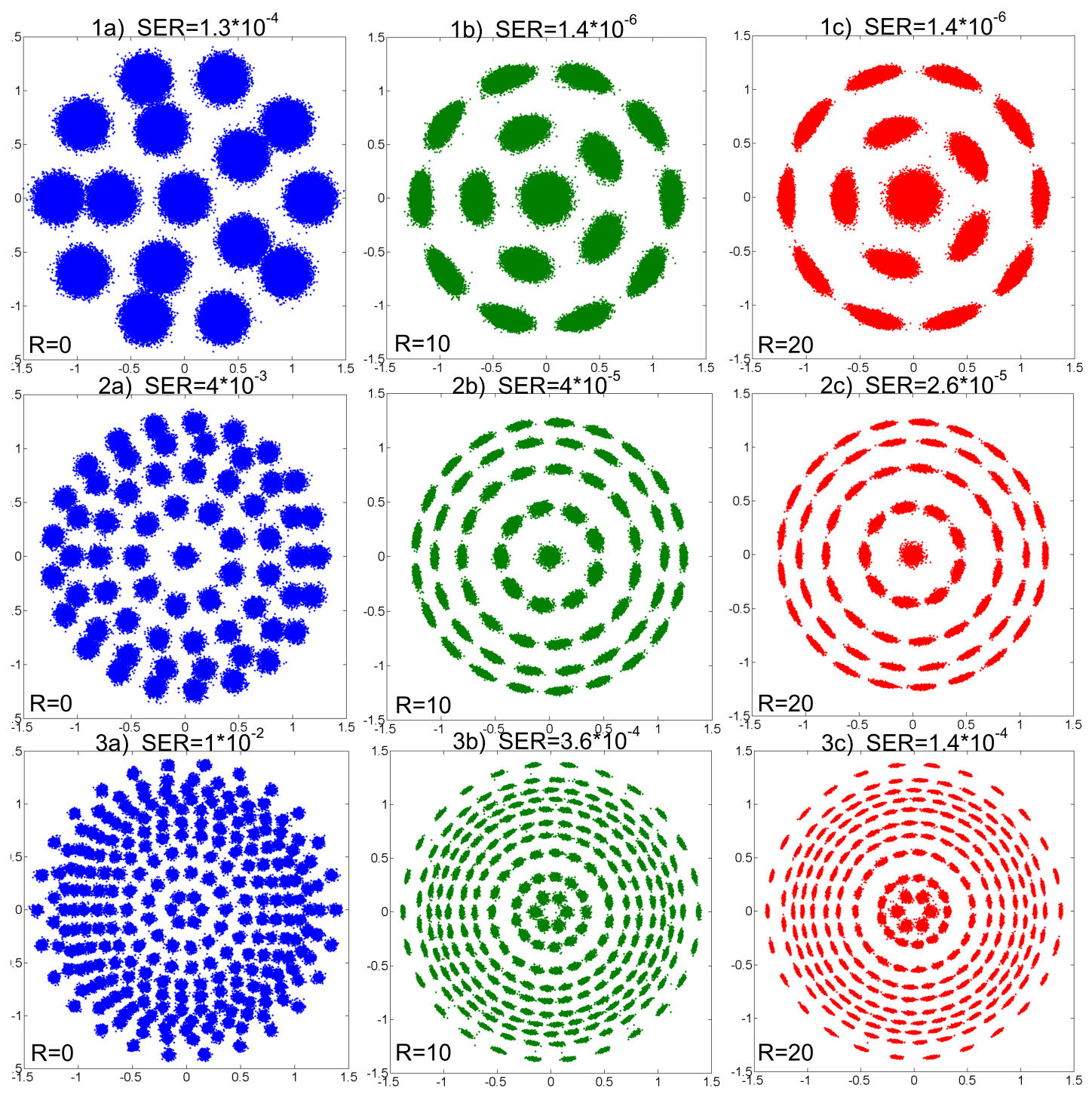

Figure 5.17: Constellation diagrams (normalized to signal power $S=1$ ) for circular (1) 16-, (2) 64- and (3) 256-QAM - after transmission (a) without and with (b)10 and (c) 20 equidistantly placed amplitude regenerators (see Fig.1 with $\gamma L=2 W^{-1}$ and $(1,2)$ $\rho_{1}=0.24, \kappa=0.03$ and (3) $\left.\rho_{1}=0.267, \kappa=0.014\right)$ ) with OPC placed in the middle of the transmission line. In the absence of regeneration, the respective linear system has (1) $\mathrm{SNR}=20 \mathrm{~dB}$, (2) $\mathrm{SNR}=25 \mathrm{~dB}$ and (3) $\mathrm{SNR}=30 \mathrm{~dB}$. 


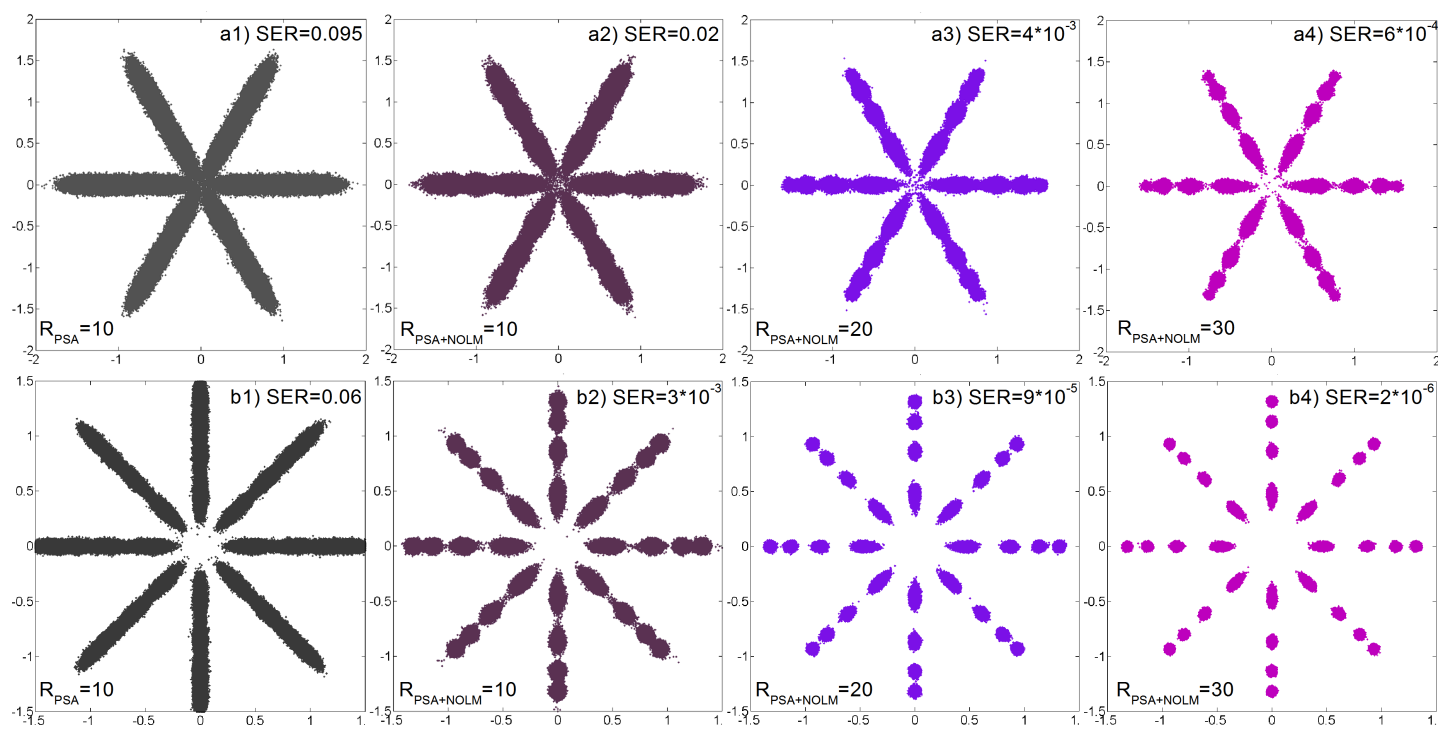

Figure 5.18: Constellation diagrams (normalized to signal power $S=1$ ) for circular a) 24- and b) 32-QAM - after transmission (1) 10 phase- and (2-4) 10, 20, 30 phase\&amplitude - regenerators equidistantly placed along the line (with parameters: $\gamma L=2 W^{-1}$ and $\rho_{1}=0.24, \kappa=0.03$ ). In the absence of regeneration, the respective linear system has (a) $\mathrm{SNR}=15 \mathrm{~dB}$ and (b) $\mathrm{SNR}=20 \mathrm{~dB}$. 
along the line. The transmission between the spans is assumed to be linear. Here we focus on the demonstration of the regenerative properties and neglect nonlinear and dispersive effects in-line. For benchmarking we use the corresponding linear system (the respective channel in the absence of regeneration) and characterize it by the signalto-noise ratio (SNR) of the linear channel. The performance was characterized by the symbol error rate (SER) obtained by direct error counting of $2^{25}$ simulated equiprobable symbols.

In the circular symbol constellations the amplitude levels were defined by the stationary and stable points of the amplitude transfer function (determined by the method of (171)), whereas the phase distribution was chosen from the analogous 16-, 64- and 256- circular QAM $(179,180,181)$. Though the constellations are non-optimal for the linear channel and in the absence of regeneration their performance is worse than the optimized circular or rectangular QAM. Nevertheless, the effective amplitude noise suppression enables us to improve performance and outperform significantly the SER of the corresponding linear channel. Further simultaneous optimization of the input format and TF parameters will increase performance. The results demonstrate the importance of optimization of signal packing for regenerative channels (182).

The power-dependent phase shift, $\delta \phi$, which, though being small, can be significant for densely packed constellations in the cascaded regime. It can be reduced by an optical phase conjugator (OPC) inserted in the middle of the transmission line (this was assumed in the simulations) or by the consequent regenerator (using the fact that the sign of the phase shift can be inverted by a different set of parameters). Alternatively, one can employ phase regenerators, which will remove the phase shift together with a reduction of the phase noise (as demonstrated in the the next section).

Figure 5.17 shows constellations at the receiver and the improvement in SER after transmission in the linear channel without regenerators (blue, left) and the regenerative channel with 10 (green, central) and 20 (red, right) regenerators. By varying parameters, we change the number of the regenerative levels and their positions. The scheme is flexible and able to handle multilevel circular formats. Increasing the number of regenerators in the cascade above the critical number leads to the saturation effect. Thus, we have illustrated that the amplitude noise is effectively squeezed, whereas the residual phase noise affects the SER. To improve further system performance, phase regeneration is required. 


\subsection{Multilevel phase and amplitude regeneration}

Next we demonstrate that the proposed scheme is compatible with phase regenerators. In particular, we consider a phase regenerator based on a phase sensitive amplifier (PSA) to be placed after each amplitude regenerator. The TF of the M-level phase regenerator (66) (cascadability of which was demonstrated in (71)) is given by (see Eq. 5.1:

$$
\sqrt{P_{\text {out }}} e^{i \varphi_{\text {out }}}=\sqrt{P_{\text {in }}} e^{i \varphi_{\text {in }}}\left(1+m e^{-i M \varphi_{\text {in }}}\right)
$$

The amplitude ratio between the signal and its $M-1$-harmonic was put to its optimal value: $m=1 /(M-1)(171)$.

Here we consider 6 and 8 level PSAs $(66,67)$. Figure 5.18 shows the received constellation diagrams after 10, 20 and 30 combined phase\&amplitude-regenerators (for reference we also plotted the received constellation after 10 PSAs). One can see that the simultaneous suppression of the amplitude and phase noise is achieved for multilevel formats.

\subsection{Regenerative Fourier Transformation}

We propose a new scheme of high efficiency regeneration of quadrature amplitude modulation (QAM) based on the regenerative Fourier transformation. Numerical modelling shows simultaneous noise suppression in both signal quadratures illustrated on 4- to 256-QAM formats.

\subsubsection{Potential implementation of RFT}

Our aim here is not to present a comprehensive analysis of implementation of the mathematical concept presented in the paper, but rather to offer a particular scheme showing a principal possibility of such transfer function. We anticipate that various practical solutions are feasible, and we aim to stimulate further discussions and research in this direction.

The proposed RFT can potentially be implemented as shown schematically in Fig. 5.19. One starts by separating two quadratures of the field (here we used a phase sensitive amplification (PSA) (see Eq. 5.1) with $m=1$ and $M=2$ (see Fig. 5.19(a)). 


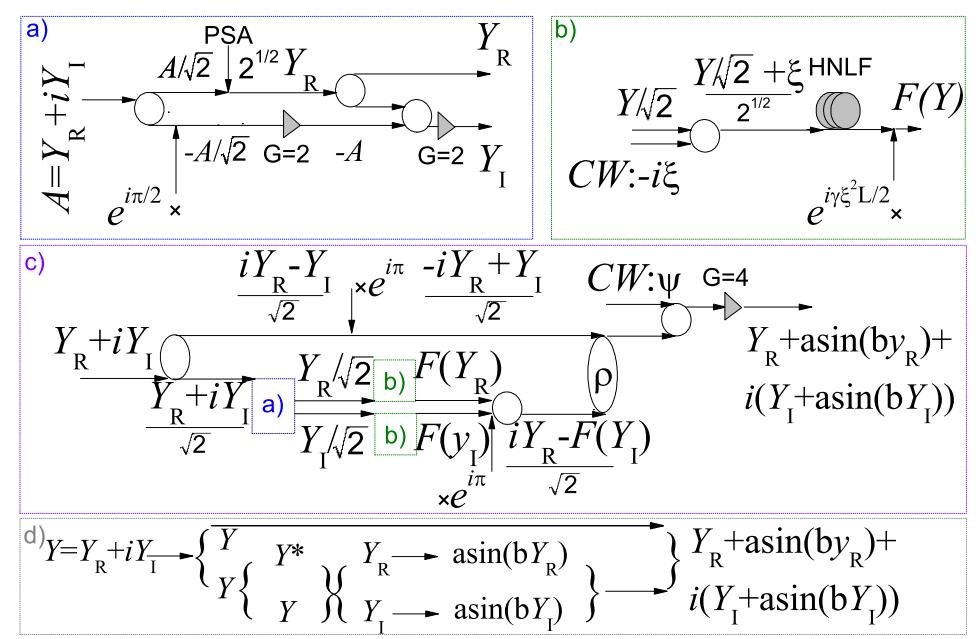

Figure 5.19: Scheme of RFT - (a) At first, we separate two quadratures of the field (denoted by $Y_{R}$ and $Y_{I}$ ); (b) then, each quadrature undergoes a nonlinear transformation, which results in the sine transformation; (c) general scheme: after the sine-transformation, the two outputs are coupled together and with the original wave; (d) simplified schematics of the proposed scheme.

Then, each of the coordinates $\left(Y_{R}, Y_{I}\right)$ is propagated through a highly nonlinear fiber (HNLF) to achieve four-wave mixing with a continuous wave $\left\langle|\xi|^{2}\right\rangle \gg\left\langle|Y|^{2}\right\rangle$, further $\xi>0$ (see Fig. 5.19(b)). Subsequently, the output is shifted by $\exp \left(i \gamma L^{2} / 2\right)$ where $\gamma$ and $L$ are nonlinear coefficient and length of HNLF, with the resulting wave:

$$
\left.F\left(Y_{(}, I\right)\right)=\frac{\xi}{\sqrt{2}} \exp \left(\frac{i \gamma L \xi Y_{R, I}}{\sqrt{2}}\right)=\frac{\xi}{\sqrt{2}}\left(\cos \left(b Y_{R, I}\right)+i \sin \left(b Y_{R, I}\right)\right)
$$

this defines a parameter $b=\gamma L \xi / \sqrt{2}$. Taking an imaginary part of $F(Y)$ will result in the sine transformation. Alternatively, with an interest in the transformation in the vicinity of the alphabet point defined by $\pi(2 k+1) / b$ where $k \in \mathrm{Z}$, one can approximate $\cos \left(b Y_{R, I}\right) \simeq 1$, which is valid up to the second order perturbation. The unity factor inside the brackets can be removed by coupling the wave with the corresponding constant wave. This procedure is applied to both quadratures simultaneously at the last coupler by using the wave: $\psi=\xi \sqrt{\rho / 4(1-\rho)}(i-1)$, where $\rho$ is the coupling parameter of the previous coupler. Once the two waves (the sine transformation of the two coordinates) have been added together, they are then coupled with the original 
wave to finally achieve the desired RFT for both quadratures (see Fig. 5.19(c-d)). All the couplers have a splitting ratio of $0.5: 0.5$ (3dB couplers), except for one coupler that has: $1-\rho: \rho$ with $\rho \ll 1$. To restore the original power, the resulted wave is amplified with the amplifier gain $G=4 /(1-\rho) \simeq 4$.

This achieves the RFT: $Y_{R, I}^{\prime}=Y_{R, I}+a \sin \left(b Y_{R, I}\right)$, with $a=\xi \sqrt{\rho / 2(1-\rho)}$ and $b=\gamma L \xi / \sqrt{2}$. It is challenging to regenerate high-order constellations (higher than 32) using the conventional approach of regenerating phase and amplitude in circular formats, as such constellations have tight phase-packing due to energy efficiency requirements. Therefore, a new approach for regenerating separately the two signal quadratures will be required. The proposed RFT is the first scheme to operate on both quadratures and enable an infinite number of regenerative levels. In this sense, this scheme will also potentially enable regeneration of the conventional rectangular (QAM) modulation formats. Moreover, being the Fourier transform of the ideal regenerator, it enables the highest regeneration efficiency without making a hard decision.

\subsubsection{Numerical Simulations}

a) $S N R=1 ; R=30$

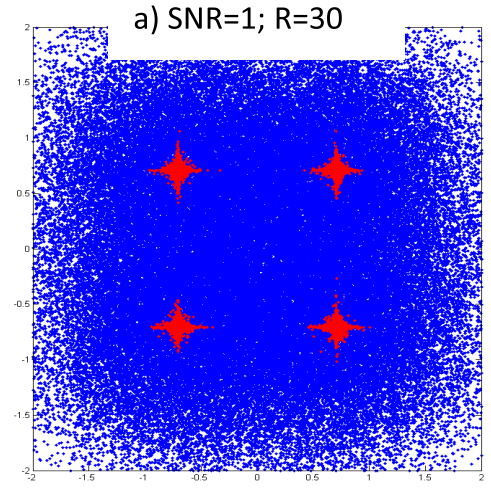

b) $S N R=5 ; R=40$

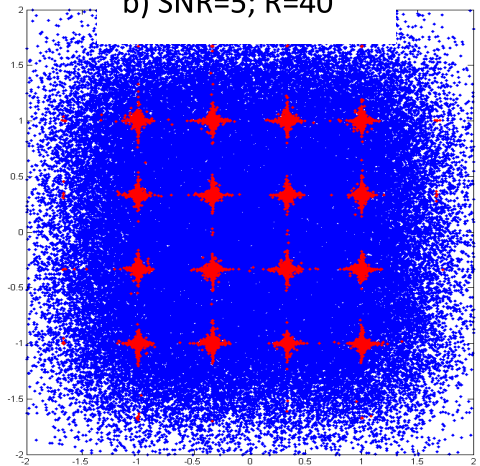

c) $S N R=10 ; R=50$

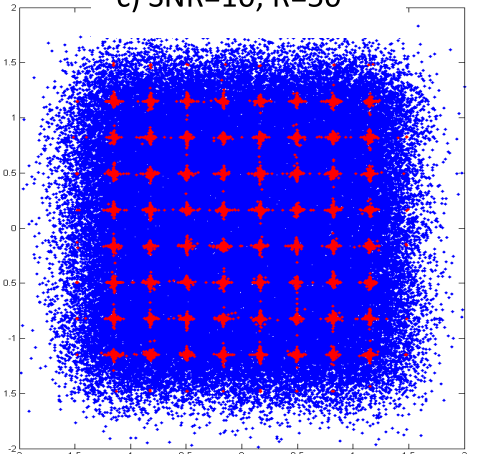

Figure 5.20: Constellation diagrams (normalized to signal power $S=1$ ) for rectangular (a) 4-, (b) 16- and (c) 64-QAM - after transmission without (blue) and with (red) $R$ equidistantly placed RFT filters with the transfer function: $Y_{R, I}^{\prime}=Y_{R, I}+a \sin \left(b Y_{R, I}\right)$. In the absence of regeneration, the corresponding linear system is characterized by the signal-to-noise ratio - SNR $(\mathrm{dB})$. 


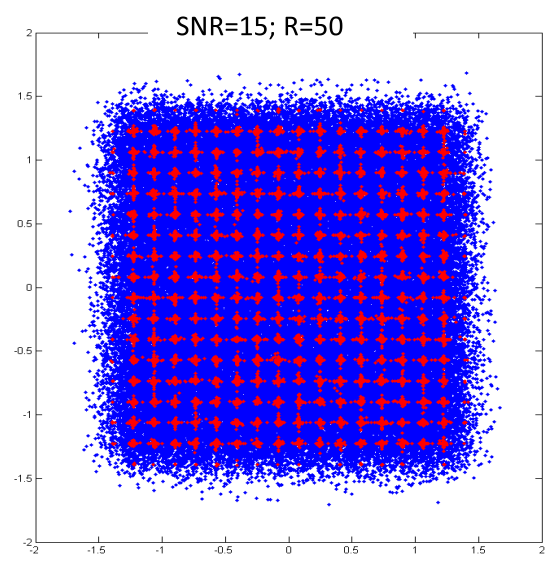

Figure 5.21: Constellation diagrams (normalized to signal power $S=1$ ) for rectangular 256-QAM - after transmission without (blue) and with (red) 50 equidistantly placed RFT filters with the transfer function: $Y_{R, I}^{\prime}=Y_{R, I}+a \sin \left(b Y_{R, I}\right)$.
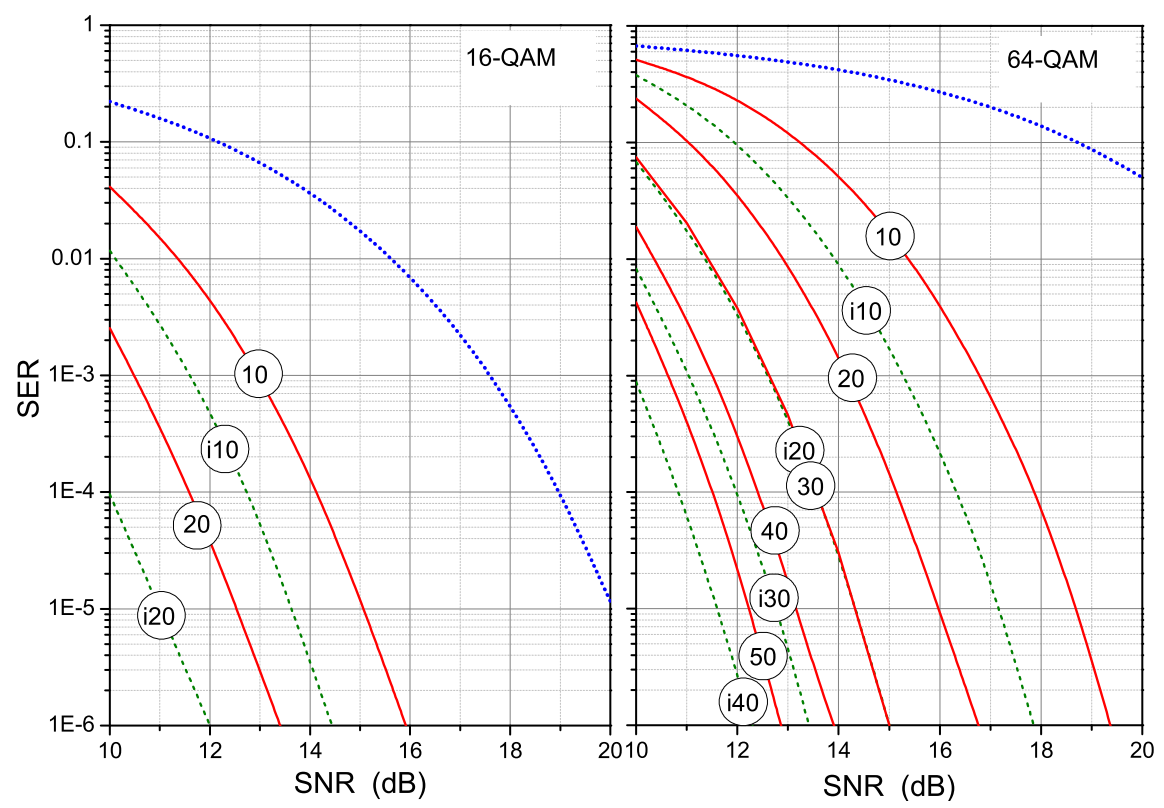

Figure 5.22: SERs as a function of SNR for 16- and 64-QAM ) for rectangular 256QAM - with a different number (placed in circles for each curve) of RFT filters with the TF: $Y_{R, I}^{\prime}=Y_{R, I}+a \sin \left(b Y_{R, I}\right)$ are shown by red solid curves. The SER of the corresponding system with the ideal regenerators are plotted by greed dashed curves (denoted by symbol i) and the SERs of the linear channel are shown for comparison by blue dotted lines. 
Next we investigated numerically the regenerative effect of the proposed scheme by simulating the transmission of rectangular 4-,16-, 64-, and 256 - QAM via noisy channel. The RFT filters were placed equidistantly along the transmission line and signal distortion was modelled as additive white Gaussian noise uniformly distributed along the line. Here, we aimed at the demonstration of the regenerative properties and considered a simplified linear transmission model, where destructive nonlinear and dispersive effects were neglected. Figure 5.20 shows constellations at the output of the last RFT filter after transmission via 30, 40, and 50 regenerators. We characterize the system by the signal-to-noise ratio (SNR) defined as the ratio of the input signal power to the power of the linearly added noise accumulated along the link, thus coinciding with the SNR of the equivalent linear system (in the absence of regeneration). One can see that the RFT filters placed in cascades enable high performance of noise suppression in both quadratures simultaneously. We plot constellations after transmission via linear system distorted by additive white Gaussian noise (depicted by blue) compared to regenerated constellations at the output of the R-th RFT filter (depicted by red). Due to the transformation the residual noise statistics is changed and the distortion is effectively reduced. The RFT is unlimited in the number of levels and can be applied to any rectangular constellation. By changing the transformation parameters (here, we varied the value of the parameter $b$ ) one can adapt the scheme for any modulation format. In Fig. 5.21 we plot the corresponding results for a rectangular 256-QAM. Thus, we demonstrate that the scheme is flexible and able to handle various constellations. Next, we calculated a symbol error rate (SER) by direct error counting of simulated equiprobable $2^{25}$ symbols as a function of the SNR for rectangular 16- and 64-QAM for different number of RFT regenerators in cascades. The results depicted in Fig. 5.22 show a dramatic improvement in SER by several orders. Moreover, the gain due to regeneration rises as the modulation complexity increases. Also, the RFT represents the highest efficiency regeneration without requirement of transponders ( $\mathrm{Tx} / \mathrm{Rx}$ ) inserted along the transmission line. Compare the SER improvement due to RFT (see Fig. 5.22, red curves) with that due to employment of the ideal regenerators (Fig. 5.22, green curves). Thus, we have demonstrated that the noise is effectively squeezed in both quadratures and the signal transmission is highly improved. 


\subsection{Conclusions}

We have proposed an analytical technique to define, in terms of a single transfer function parameter, the optimum conditions and operating margins of cascaded PSA based regenerators in multi-level phase encoded transmission links. Extensive numerical simulations have been carried out and have confirmed the analytical predictions of our theory. Furthermore, they have revealed the performance dynamics along the cascaded system. It has been found that all-optical phase regeneration brings significant performance gain to the system, when moving to larger signal complexities, but reduces the operating margins. A saturation effect in performance improvement has been also predicted, which evolves more rapidly for non-optimal transfer functions and high modulation orders. The developed theory will be essential for the design of future high capacity transmission systems with all-optical regenerators.

Next, we have investigated the transmission performance of non-linear regenerative channels based on cascaded PSAs and we have explored the impact of the phase and amplitude noise accumulation mechanisms in the selection of the optimum modulation format. The results demonstrate that densely packed PSK formats are highly favored by the phase squeezing properties of the channel for high capacity transmission. On the other hand, the use of multi-amplitude level ring constellations brings minor improvements in SER performance as the benefits of phase regeneration are counterbalanced by the enhancement of the amplitude noise imposed by the phase to amplitude conversion in the PSAs. In particular, we find that the use of PSA regeneration with a 16-PSK signal (with 20 PSA-based regenerators) outperforms the linear transmission performance of the 16-QAM system by $20 \mathrm{~dB}$ (4 order o magnitude) in SER at an SNR of $16 \mathrm{~dB}$, suggesting that a system employing phase regenerators has the potential to offer higher point to point capacities than conventional dual quadrature transmission systems.

Also, we have proposed a novel scheme for multilevel amplitude regeneration and demonstrated effective amplitude noise suppression for 9 (or more) amplitude levels. The design is simple and flexible, where by varying parameters one can change the number of levels and adjust their positioning. The model was illustrated on circular 16-, 64- and 256-QAM. Combined with PSAs, the simultaneous suppression of both 
phase and amplitude noise was achieved and demonstrated on circular 16- and 32QAM.

Finally, we demonstrated regeneration of conventional multilevel rectangular QAM with effective noise suppression in both quadratures simultaneously. The design is simple and flexible, by varying transfer function parameters one can adapt a design for any modulation format. The cascadability of the model was illustrated on rectangular 4-, 16-, 64-, and 256-QAM. 


\section{6}

\section{Conclusion and discussion}

Future communication systems are expected to combine advanced technological innovations in an integrated and optimized way to ensure high capacity transmission. The ever-increasing capacity demand resulted in the surge of new techniques that appeared over the decade. Thus, new technology led to new channel types with different properties: stochastic (e.g. noise source due to EDFA or Raman amplification) and deterministic, both destructive (due to Kerr effect or mode coupling in optical fibers) and constructive (regeneration based on nonlinear signal transformations).

These created a new field of information theory research with a vast area of applications. However, calculating the Shannon capacity for nonlinear channels is limited since, for a number of channels, the conditional pdf was not derived. As a result, instead of the Shannon capacity (the maximum limit of zero error transmission rate), a number of approximate estimations were made.

We derived the Shannon capacity for regenerative channels. We adapted the mathematical method - stability analyses of dynamical systems to regeneration. This resulted in a simple, general and analytical scheme - regenerative mapping, which consists of the system of three equations. Further, applied to the existing regenerative phase quantizer scheme (practically realized by PSAs), the method showed the excellent agreement with the numerical simulations and defined the optimal parameter value and operating margins of PSAs.

Furthermore, we proposed the model of the regenerative Fourier transform (RFT), which is the closest continuous approximation of the ideal regenerator. This can be 
further used for benchmarking different regenerative schemes. RFT, being general and based on FT, enables efficient regeneration of multilevel, multidimensional signals.

Hence, the analytical procedure for optimizing arbitrary regenerative elements was derived and thoroughly verified numerically. Using it, we have developed a method for calculating the conditional pdf that characterizes a regenerative channel and is necessary for the capacity calculation. Here, we generalized the result of (72), where the conditional pdf for the system with the ideal regenerators was presented. The method proposed here is suitable for calculating the conditional pdf for arbitrary regenerative models with smooth transfer functions.

Next, using the obtained result, we calculated the Shannon capacity of the RFT nonlinear channel. Moreover, we calculated the analytical asymptotical formula for the Shannon capacity at high SNR.

Also, we calculated the analytical upper bound of regeneration efficiency - the maximum Shannon capacity achieved by regeneration. The corresponding capacity gain due to regeneration was derived.

Furthermore, we showed that by optimizing a regenerative system in accordance with the proposed regenerative mapping technique, one can approach the derived regenerative limit at high SNR. This was demonstrated and proved on the example of the RFT channel.

Also, we demonstrated the importance of optimization in the regenerative channel by studying the efficiency of different modulation formats. It was shown that without optimization, regeneration can degrade system performance; whereas accurately optimized systems can result in capacity a few times higher than the corresponding linear limit. This was further demonstrated on the example of the regenerative system with cascaded PSAs, where the optimal packing was proved to be a densely packed PSK format, which in the case of regenerated cascaded PSAs outperform the conventional multilevel QAM, suboptimal in linear Gaussian channels.

Finally, we proposed a novel scheme for multilevel amplitude regeneration based on a nonlinear optical loop mirror (NOLM) and demonstrated through numerical modelling its efficiency and cascadability on circular 16-, 64- and 256-symbol constellations. We showed that the amplitude noise is efficiently suppressed. The design is flexible and enables varying the number of levels and their positioning. The scheme is compatible with phase regenerators. Also, compared to the traditional scheme of one 
NOLM, new features, such as reduced and sign-varied power-dependent phase shifts, are available in the proposed scheme. The model requires only two couplers in addition to the traditional NOLM, and offers a vast range of optimization parameters.

Overall, we calculated the Shannon capacity of different communication channels with constructive regenerative nonlinearity. Novel results of the Shannon capacity for the new channel types were obtained with the emphasis on optimizing input distribution and the regenerative element. The described effects lead to new and important insights into the nature and characteristics of nonlinear information channels. We believe that the work will open new possibilities for information transmission leading towards the revaluation of the fundamental nonlinear Shannon limit. 


\section{7 \\ List of publications related to the thesis}

1. M. A. Sorokina and S. K. Turitsyn, "Regeneration limit of classical Shannon capacity," Nat. Comm. 5 (2014). (ch. 5).

2. M. A. Sorokina, "Design of multilevel amplitude regenerative system," Opt. Letters 39, 2499-2502 (2014) (ch. 6).

3. M. Sorokina, S. Sygletos, A. D. Ellis, and S. Turitsyn, "Optimal packing for cascaded regenerative transmission based on phase sensitive amplifiers," Opt. Express 21, 31201-31211 (2013) (ch. 6).

4. M. A. Sorokina, S. Sygletos, and S. K. Turitsyn, "Optimization of cascaded regenerative links based on phase sensitive amplifiers", Opt. Letters 38, 43784381 (2013) (ch. 6).

5. A.D. Ellis, M. Sorokina, S. Sygletos, S.K. Turitsyn, "Capacity in nonlinear fiber transmission systems", ACP2013 (invited) (ch. 5).

6. M. Sorokina, and S. Turitsyn, "Design of nonlinear regenerative systems with high capacity", ICTON2013 (invited) (ch. 5).

7. M. A. Sorokina and S. K. Turitsyn, "Nonlinear signal transformations: Path to capacity above linear channels," 2014 IEEE Summer Topical Meeting on Nonlinear-Optical Signal Processing (invited) (ch. 5). 
8. M. A. Sorokina and S. K. Turitsyn, "Efficiency of different regenerative channels," ICTON 2014 (invited) (ch. 6).

9. M. Sorokina, S. Sygletos, and S. Turitsyn, "Optimization method for PSA-based multi-level regenerators", ECOC 2013 (P.4.7) (ch. 6).

10. M. Sorokina, S. Sygletos, and S. Turitsyn, "Efficient packing for phase regenerative channels", IPC2013 (ch. 6).

11. M. A. Sorokina, "Multilevel amplitude regeneration of 256-symbol constellation" CLEO 2014 (ch. 6).

12. S. Sygletos, M. E. McCarthy, S. J. Fabbri, M. Sorokina, M. F. C. Stephens, I. D. Phillips, E. Giacoumidis, N. Mac Suibhne, P. Harper, N. J. Doran, S. K. Turitsyn, A. D. Ellis, "Multichannel regeneration of dual quadrature signals," ECOC 2014 (accepted) (ch 6). 


\section{Bibliography}

[1] C. E. Shannon, "Mathematical theory of communication," Bell Syst. Tech. J. 27, 379 (1948); 27, 623 (1948).

[2] Keang-Po Ho, "Exact evaluation of the capacity for intensity-modulated directdetection channels with optical amplifier noises", IEEE Photonics Technology Letters, 174, 858-860 (2005).

[3] M. Katz and S. Shamai (Shitz), "On the capacity-achieving distribution of the discrete-time noncoherent and partially coherent AWGN channels," IEEE Trans. Inf. Theory, 50 (10), 2257-2270 (2004).

[4] P. Hou, B. Belzer, and T. R. Fischer, "Shaping gain of partially coherent additive white Gaussian noise channel," in Proc. Conf. Information Sciences and Systems, IEEE Commun. Lett., 6, 175-177, May 2002.

[5] S. Verdu and T. S. Han, "A general formula for channel capacity," IEEE Trans. Inf. Theory, 40(4), 1147-1157 (1994).

[6] E. E. Narimanov and P. Mitra, "The channel capacity of a fiber optics communication system: perturbation theory," J. Lightw. Technol., 20 (3) 530-537 (2002).

[7] J. Tang, "The Shannon channel capacity of dispersion-free nonlinear optical fiber transmission," J. Lightw. Technol., 19(8), 1104- 1109 (2001).

[8] J. Tang, "A comparison study of the Shannon channel capacity of various nonlinear optical fibers," J. Lightw. Technol., 24(5), 2070- 2075 (2006). 
[9] B. Goebel, R. Essiambre, G. Kramer, P.J. Winzer, N. Hanik, "Calculation of Mutual Information for Partially Coherent Gaussian Channels With Applications to Fiber Optics", IEEE Transactions on Information Theory, 57 (9), 5720 - 5736 (2011).

[10] E. Agrell, M. Karlsson, "Satellite constellations: Towards the nonlinear channel capacity", Photonics Conference (IPC), 316 - 3172012 IEEE.

[11] A. Splett, C. Kurzke, and K. Petermann, In Proc. of the European Conf. on Opt. Com., ECOC93, 2, 41 (1993).

[12] P. P. Mitra and J. B. Stark, "Nonlinear limits to the information capacity of optical fibre communications," Nature 411, 1027-1030 (2001).

[13] J. B. Stark, P. Mitra, A. Sengupta, "Information capacity of nonlinear wavelength division multiplexing fiber optic transmission line," Optical Fiber Technology 7, 275-288 (2001).

[14] X. Chen and W. Shieh, "Closed-form expressions for nonlinear transmission performance of densely spaced coherent optical OFDM systems," Opt. Express 18, 19039-19054 (2010)

[15] R.-J. Essiambre, G. J. Foschini, G. Kramer, and P. J.Winzer, "Capacity limits of optical fiber networks," Phys. Rev. Lett., 101, 163901 (2008).

[16] R. J. Essiambre, G. Kramer, P. J. Winzer, G. J. Foschini, and B. Goebel, "Capacity limits of optical fiber networks," J. Lightwave Technol. 28(4), 662-701 (2010).

[17] K.S. Turitsyn, S.A. Derevyanko, I.V. Yurkevich, and S. K. Turitsyn, "Information capacity of optical fiber channels with zero average dispersion," Physical Review Letters 91, 203901 (2003).

[18] A.D. Ellis, J. Zhao, and D. Cotter, "Approaching the non-linear Shannon limit," J. Lightwave Technol. 28, 423-433(2010).

[19] E. Agrell and M. Karlsson, "WDM channel capacity and its dependence on multichannel adaptation models", Optical Fiber Communication Conference and 
Exposition and the National Fiber Optic Engineers Conference, OFC/NFOEC (2013).

[20] J. Wang, J.-Y. Yang, I. M. Fazal, N. Ahmed, Y. Yan, H. Huang, Y. Ren, Y. Yue, S. Dolinar, M. Tur, and A. E. Willner, "Terabit free-space data transmission employing orbital angular momentum multiplexing," Nature Photonics 6, 488-496 (2012).

[21] R. Konig and G. Smith, "Limits on classical communication from quantum entropy power inequalities," Nature Photonics 7, 142-146 (2013).

[22] P. J. Winzer and R.-J. Essiambre, "Advanced modulation formats for highcapacity optical transport networks", J. of Light. Technol 24, 4711-4728 (2006).

[23] R. J. Essiambre, G. Kramer, P.J. Winzer, G.J. Foschini, B. Goebel, "Capacity Limits of Optical Fiber Networks," J. Lightwave Technol. 28, 662-701 (2010).

[24] Z. H. Peric, I. B. Djordjevic, S. M. Bogosavljevic, and M. C. Stefanovic, "Design of signal constellations for Gaussian channel by iterative polar quantization," in Proc. 9th Mediterranean Electrotech. Conf., May 1998, vol. 2, 866-869.

[25] H.G. Batshon, I.B. Djordjevic, L. Xu, and T. Wang, "Iterative polar quantizationbased modulation to achieve channel capacity in ultrahigh-speed optical communication systems", IEEE Photonics Journal, 2(4), 593 - 5992010.

[26] I.B. Djordjevic, "Spatial-domain-based hybrid multidimensional codedmodulation schemes enabling multi-Tb/s optical transport", Journal of Lightw. Technol., 30(14), 2315 - 2328 (2012).

[27] T.H. Lotz, X. Liu, S. Chandrasekhar, P.J. Winzer, H. Haunstein, S. Randel, S. Corteselli, B. Zhu, D.W. Peckham, "Coded PDM-OFDM transmission with shaped 256-iterative-polar-modulation achieving 11.15-b/s/Hz intrachannel spectral efficiency and 800-km reach", Journal of Lightwave Technology, 31(4), 538 - 545 (2013).

[28] M. Karlsson and E. Agrell, "Power-efficient modulation schemes" in Impact of Nonlinearities on Fiber Optic Communications, Optical and Fiber Communications Reports, 7, 219-252 (2011). 
[29] K. C. Kao and G. A. Hockham, "Dielectric-fibre surface waveguides for optical frequencies", Proc. IEEE 113, 1151-1158 (1966).

[30] D. J. Richardson, "Filling the pipe", Science 330, 327 (2010).

[31] D.J. Malyon, T. Widdowson, E.G. Bryant, S.F. Carter, J.V. Wright, W.A. Stallard, Electron. Lett. 27(2), 120-121 (1991)

[32] H.J. Thiele, R.I. Killey, and P. Bayvel, "Influence of fibre dispersion and bit rate on cross-phase-modulation-induced distortion in amplified optical fibre links," Electron. Lett. 34(21), 2050-2051 (1998).

[33] A.D. Ellis and W.A. Stallard, "Four wave mixing in ultra long transmission systems incorporating linear amplifiers", IEE Colloquium, 159 (1990), http://ieeexplore.ieee.org/xpl/freeabs all.jsp? arnumber=190875

[34] R.-J. Essiambre, B. Mikkelsen, G. Raybon, "Intra-channel cross-phase modulation and four-wave mixing in high-speed TDM systems," Electron. Lett. 35(18), 1576-1578 (1999).

[35] A.D. Ellis, J.D. Cox, D. Bird, J. Regnault, J.V. Wright, W.A. Stallard, "5 Gbit/s soliton propagation over $350 \mathrm{~km}$ with large periodic dispersion coefficient perturbations using erbium doped fibre amplifier repeaters," Electron. Lett. 27(10), 878 (1991).

[36] I. Morita, K. Tanaka, N. Edagawa, M. Suzuki, "Impact of the dispersion map on long-haul 40 Gbith single-channel soliton transmission with periodic dispersion compensation", in Proceedings of OFC99, San Diego, Paper FD1, 1999

[37] P.V. Mamyshev and L.F. Mollenauer, "Pseudo-phase-matched four-wave mixing in soliton wavelength-division multiplexing transmission," Opt. Lett 21(6), 396398 (1996).

[38] N.J. Smith and N.J. Doran, "Modulational instabilities in fibers with periodic dispersion management," Opt. Lett. 21(8), 570-572 (1996). 
[39] E Pincemin, A. Tan, A. Bezard, A. Tonello, S.Wabnitz, J-D Ania-Castanon, S. Turitsyn, "Robustness of $40 \mathrm{~Gb} / \mathrm{s}$ ASK modulation formats in the practical system infrastructure," Opt. Express 14(25), 12049-12062 (2006).

[40] S. Watanabe, S. Kaneko, T. Chikama, "Long-haul fibertransmission using optical phase conjugation", Optical Fiber Technology, 2(2), 169-178 (1996).

[41] M. Nakazawa,E. Yamada, H. Kubota, K. Suzuki , "10 Gbit/s soliton data transmission over one million kilometres," Electronics Letters, 27(14), 1270-1272 (1991).

[42] F. Seguineau, B. Lavigne, D. Rouvillain, P. Brindel, L. Pierre, and O. Leclerc, "Experimental demonstration of simple NOLM-based 2R regenerator for 42.66 Gbit/sWDMlong-haul transmissions," Proc. Opt. Fiber Commun. Conf. WN4 (2004).

[43] A. Gray, Z.Huang, I. Khrushchev, and I. Bennion, "Autosoliton propagation at 80 Gbit/s using concatenated nonlinear loop switches in standard fibre," Electron. Lett., 40(8), 498-500 (2004).

[44] G. Goldfarb, M.G. Taylor, G. Li; , "Experimental demonstration of fiber impairment compensation using the split-step infinite impulse response method", Digest of the IEEE/LEOS Summer Topical Meetings 2008, 145-146, (2008).

[45] D. Rafique, J. Zhao, and A. D. Ellis, "Digital back-propagation for spectrally efficient WDM 112 Gbit/s PM m-ary QAM transmission," Opt. Express 19, 52195224 (2011).

[46] E. Agrell, "The channel capacity increases with power", arXiv:1108.0391.

[47] P. Poggiolini, A. Carena, V. Curri, G. Bosco, and F. Forghieri, "Analytical modeling of non-linear propagation in uncompensated optical transmission links," IEEE Photon. Technol. Lett. 23, 742-744 (2011).

[48] J. Sakaguchi, Y. Awaji, N. Wada, A. Kanno, T. Kawanishi, T. Hayashi, T. Taru, T. Kobayashi, and M. Watanabe, "Space division multiplexed transmission of 109$\mathrm{Tb} / \mathrm{s}$ data signals using homogeneous seven-core fiber," J. Lightwave Technol. 30, 658-665 (2012). 
[49] R. Ryf, S. Randel, A. H. Gnauck, C. Bolle, A. Sierra, S. Mumtaz, M. Esmaeelpour, E. C. Burrows, R.-J. Essiambre, P. J. Winzer, D. W. Peckham, A. H. McCurdy, and R. Lingle, "Mode-division multiplexing over $96 \mathrm{~km}$ of fewmode fiber using coherent $6 \times 6$ MIMO processing," J. Lightwave Technol. 30, 521-531 (2012).

[50] A, Mecozzi, C, Antonelli, and M, Shtaif, "Nonlinear propagation in multi-mode fibers in the strong coupling regime," Opt. Express 20, 11673-11678 (2012)

[51] R. A. Fisher, B. R. Suydam, and D. Yevick, "Optical phase conjugation for timedomain undoing of dispersive self-phase-modulation effects," Opt. Lett. 8, 611613 (1983).

[52] A. H. Gnauck, R. M. Jopson, and R. M. Derosier, "10-Gb/s 360-km transmission over dispersive fiber using midsystem spectral inversion," IEEE Photon. Technol. Lett. 5, 663-666 (1993).

[53] S. Watanabe, T. Chikama, G. Ishikawa, T. Terahara, and H. Kuwahara, "Compensation of pulse shape distortion due to chromatic dispersion and Kerr effect by optical phase conjugation," IEEE Photon. Technol. Lett. 5, 1241-1243 (1993).

[54] X. Chen, X. Liu, S. Chandrasekhar, B. Zhu, and R. W. Tkach, "Experimental demonstration of fiber nonlinearity mitigation using digital phase conjugation," Optical Fiber Communication Conference (OFC), paper OTh3C.1 (2012).

[55] M. D. Pelusi and B. J. Eggleton, "Optically tunable compensation of nonlinear signal distortion in optical fiber by end-span optical phase conjugation," Opt. Express 20, 8015-8023 (2012).

[56] D. Rafique and A. D. Ellis, "Nonlinearity compensation in multi-rate 28 Gbaud WDM systems employing optical and digital techniques under diverse link configurations," Opt. Express 19, 16919-16926 (2011).

[57] X. Liu, A. R. Chraplyvy, P. J. Winzer, R. W. Tkach, and S. Chandrasekhar, "Phase-conjugated twin waves for communication beyond the Kerr nonlinearity limit," Nature Photonics 7, 560-568 (2013). 
[58] E. Ip and J. M. Kahn, "Fiber communications: time-reversed twin," Nature Photonics 7, 507-508 (2013).

[59] X. Li, X. Chen, G. Goldfarb, E. Mateo, I. Kim, F. Yaman, and G. Li, "Electronic post-compensation of WDM transmission impairments using coherent detection and digital signal processing," Opt. Express 16(2), 880-888 (2008).

[60] E. G. Turitsyna and S. K. Turitsyn, "Digital signal processing based on inverse scattering transform," Opt. Lett. 38, 4186-4188 (2013).

[61] M. Matsumoto, "Fiber-based all-optical signal regeneration," IEEE J. Sel. Top. Quantum Electron., 18(2), 738-752 (2012).

[62] S. Sygletos, P. Frascella, S.K. Ibrahim, L. Gruner-Nielsen, R. Phelan, J. O"Gorman, A.D. Ellis "A practical phase sensitive amplification scheme for two channel phase regeneration", Optics Express 19(26), B938-B945 (2011).

[63] R. Slavik, F. Parmigiani, J. Kakande, C. Lundstrom, M. Sjodin, P. Andrekson, R. Weerasuriya, S. Sygletos, A. D. Ellis, L. Gruner-Nielsen, D. Jakobsen, S. Herstrom, R. Phelan, J. O'Gorman, A. Bogris, D. Syvridis, S. Dasgupta, P. Petropoulos, and D. Richardson, "All-optical phase and amplitude regenerator for next generation telecommunications systems," Nat. Photonics 4(10), 690-695 (2010).

[64] R. P. Webb, J. M. Dailey, R. J. Manning, and A. D. Ellis, "Phase discrimination and simultaneous frequency conversion of the orthogonal components of an optical signal by four-wave mixing in an SOA," Optics Express 19, 20015-20022 (2011).

[65] S. Sygletos, M. E. McCarthy, S. J. Fabbri, M. Sorokina, M. F. C. Stephens, I. D. Phillips, E. Giacoumidis, N. Mac Suibhne, P. Harper, N. J. Doran, S. K. Turitsyn, A. D. Ellis, "Multichannel regeneration of dual quadrature signals," in Optical Communication (ECOC 2014), 39th European Conference and Exhibition on Optical Communications, P.3.13. 
[66] J. Kakande, R. Slavik, F. Parmigiani, A. Bogris, D. Syvridis, L. Gruner-Nielsen, R. Phelan, P. Petropoulos, and D. J. Richardson, "Multilevel quantization of optical phase in a novel coherent parametric mixer architecture," Nat. Photonics 4(12), 748-752 (2011).

[67] G. Hesketh and P. Horak, "Reducing bit-error rate with optical phase regeneration in multilevel modulation formats," Opt. Lett. 38, 5357-5360 (2013).

[68] A. Bogris and D. Syvridis, "All-optical signal processing for 16-QAM using fourlevel optical phase quantizers based on phase sensitive amplifiers," in Proceedings of European Conference in Optical Communications (ECOC 2013), London, paper We.3A.6, (2013).

[69] F. Seguineau, B. Lavigne, D. Rouvillain, P. Brindel, L. Pierre, and O. Leclerc, "Experimental demonstration of simple NOLM-based 2R regenerator for 42.66 Gbit/sWDMlong-haul transmissions," Proc. Opt. Fiber Commun. Conf. WN4 (2004).

[70] A. Gray, Z.Huang, I. Khrushchev, and I. Bennion, "Autosoliton propagation at 80 Gbit/s using concatenated nonlinear loop switches in standard fibre," Electron. Lett., 40(8), 498-500 (2004).

[71] T. Umeki, M. Asobe, H. Takara, Y. Miyamoto, and H. Takenouchi, "Multispan transmission using phase and amplitude regeneration in PPLN-based PSA," 21(15), 18170-18177 (2013).

[72] K. S. Turitsyn, K.S and S.K. Turitsyn, "Nonlinear communication channels with capacity above the linear Shannon limit," Opt. Lett. 37, 3600-3602 (2012).

[73] J. Gleck, The Information: A History, a Theory, a Flood (Fourth Estate, 2011).

[74] J. G. Proakis, Digital Communications (McGraw-Hill, New York, 2001).

[75] H. Nyquist, "Certain factors affecting telegraph speed," Bell Syst. Tech. J., 3, 324-352 (1924).

[76] H. Nyquist, "Certain topics in telegraph transmission theory," AIEE Trans., 47, 617-644 (1928). 
[77] http://www.quantium.plus.com/ahr/patents.htm

[78] K. Kupfmuller, "Uber Einschwingvorgange in Wellenfiltern," Elek. Nachrichtentech., 1, 141-152 (1924).

[79] V. A. Kotelnikov, "On the transmission capacity of "ether" and wire in electrocommunications," Izd. Red. Upr. Svyazi RKKA (Moscow, USSR) (material for the first all-union conference on questions of communications), 44 (1933).

[80] E. T. Whittaker, "On the functions which are represented by the expansion of interpolating theory," in Proc. Roy. Soc. Edinburgh, 35, 181-194 (1915).

[81] J. M. Whittaker, "The Fourier theory of the cardinal functions," Proc. Math. Soc. Edinburgh, 1, 169-176 (1929).

[82] R. V. L. Hartley, "Transmission of information," Bell Syst. Tech. J., 7, 535-563 (1928).

[83] C. E. Shannon, "A mathematical theory of cryptography," Tech. Rep. MM 45110-02, Bell Labs. Tech. Memo., (1945).

[84] S. Verdu, "Fifty years of Shannon theory," IEEE Trans. Information Theory, Special Commemorative Issue, 44 6, 2057-2078 (1998).

[85] C. E. Shannon, "Communication theory of secrecy systems," Bell Syst. Tech. J., 28, 656-715 (1949).

[86] D. Gabor, "Theory of communication," J. Inst. Elec. Eng., 93, 429-457 (1946).

[87] Heisenberg, W. (03/01/1927). "Uber den anschaulichen Inhalt der quantentheoretischen Kinematik und Mechanik". Zeitschrift fur Physik 43 (3-4): 172-198, 1927.

[88] J. von Neumann. Mathematical foundations of quantum mechanics. Princeton University Press, Princeton, NJ, 1932, 1949, 1955.

[89] N. Wiener, Cybernetics, (New York: Wiley, 1948). 
[90] L. Boltzmann, "Beziehung zwischen dem Zweiten Hauptsatze der Mechanischen Waermertheorie und der Wahrscheilichkeitsrechnung Respektive den Saetzen uber das Waermegleichgwicht," Wien. Ber., 76, 373-435 (1877).

[91] R. A. Fisher, "Probability, likelihood and quantity of information in the logic of uncertain inference," Proc. Roy. Soc. London, A, 146, 1-8 (1934).

[92] T. M. Cover and J.A. Thomas, Elements of Information Theory, (Wiley, 1991)

[93] D. J. MacKay, Information theory, interference, and learning algorithms, (Cambridge university press, 2009).

[94] C. E. Shannon, "Coding theorems for a discrete source with a fidelity criterion," in IRE Nat. Conv. Rec., 142-163, (1959).

[95] W. Karush, Minima of Functions of Several Variables with Inequalities as Side Constraints. M.Sc. Dissertation. Dept. of Mathematics, Univ. of Chicago,Chicago, Illinois (1939).

[96] H. W. Kuhn and A. W. Tucker, "Nonlinear programming". Proceedings of 2nd Berkeley Symposium. Berkeley: University of California Press. 481-492 (1951).

[97] M. Frank and P. Wolfe, "An algorithm for quadratic programming", Naval Research Logistics Quarterly 3: 95. doi:10.1002/nav.3800030109 (1956).

[98] S. Arimoto, "An algorithm for computing the capacity of arbitrary discrete memoryless channels," IEEE T. Inform Theory, 18, 14-20 (1972).

[99] R. Blahut, "Computation of channel capacity and rate-distortion functions," IEEE T. Inform Theory, 18, 460-473 (1972).

[100] G. Matz and P. Duhamel, "Information geometric formulation and interpretation of accelerated Blahut-Arimoto-type algorithms," Proc. 2004 IEEE Information Theory Workshop, San Antonio, TX, USA, Oct. 24-29, 2004.

[101] N. Varnica, X. Ma, and A. Kavcic , "Capacity of power constrained memoryless AWGN channels with fixed input constellations," in Proc. IEEE Global Telecommunications Conf. (GLOBECOM), Taipei, Taiwan, China, Nov. 2002, pp. 1339-1343. 
[102] C.-I. Chang and L. D. Davisson, "On calculating the capacity of an infinite-input finite (infinite) -output channel," IEEE Trans. Inf. Theory, 34, 1004-1010 (1988).

[103] J. Dauwels, "Numerical computation of the capacity of continuous memoryless channels," in Proc. Symposium on Information Theory in the Benelux, Brussels, Belgium, May 2005, pp. 221-228.

[104] D. Arnold and H.-A. Loeliger, "On the information rate of binary-input channels with memory," Proc. 2001 IEEE Int. Conf. on Communications, Helsinki, Finland, June 11âĂŞ14, 2001, pp. 2692-2695.

[105] V. Sharma and S. K. Singh, "Entropy and channel capacity in the regenerative setup with applications to Markov channels," Proc. 2001 IEEE Int. Symp. Information Theory, Washington, DC, USA, June 24-29, 2001, p. 283.

[106] H. D. Pfister, J. B. Soriaga, and P. H. Siegel, "On the achievable information rates of finite-state ISI channels," Proc. 2001 IEEE Globecom, San Antonio, TX, pp. 2992-2996, Nov. 25-29, 2001.

[107] J. Dauwels and H.-A. Loeliger, "Computation of information rates by particle methods," IEEE Trans. Inf. Theory, 54, 406-409, (2008).

[108] G.P. Agrawal, Fiber-Optic Communication Systems, 3rd ed (Wiley, New York, 2002).

[109] J. Rice, Mathematical Statistics and Data Analysis (Second ed.), (Duxbury Press, ISBN 0-534-20934-3, 1995).

[110] C. E. Shannon, "The zero error capacity of a noisy channel," IRE Trans. Inform. Theory, vol. IT-2, 112-124 (1956).

[111] J. Kerr, "A new relation between electricity and light: Dielectrified media birefringent," Phil. Mag., 5019, 3337 (1875).

[112] James Clerk Maxwell, "A Dynamical Theory of the Electromagnetic Field", Philosophical Transactions of the Royal Society of London 155, 459-512 (1865). 
[113] E. Iannone, F. Matera, A. Mecozzi, and M. Settembre, Nonlinear Optical Communication Networks (Wiley, 1998).

[114] S. G. Evangelides, L. F. Mollenauer, J. P. Gordon, and N. S. Bergano, "Polarization multiplexing with solitons," J. Lightw. Technol., 10(1), 28-35 (1992).

[115] A. Einstein, "On the quantum theory of radiation," Phys. Zeits., 18121 (1917).

[116] C. W. Gardiner and P. Zoller, Quantum Noise: A Handbook of Markovian and Non-Markovian Quantum Stochastic Methods With Applications to Quantum Optics, 3rd ed. New York: Springer-Verlag, 2004.

[117] J. P. Gordon, W. H. Louisell, and L. R. Walker, "Quantum fluctuations and noise in parametric processes II," Phys. Rev., 129, 481-485 (1963).

[118] J. P. Gordon, L. R.Walker, and W. H. Louisell, "Quantum statistics of masers and attenuators," Phys. Rev., 130, 806-812 (1963).

[119] S. V. Manakov, "On the theory of two-dimensional stationary self-focusing of electromagnetic waves," Sov. Phys. JETP, 38, 248-253 (1974).

[120] D. Marcuse, C. R. Menyuk, and P. K. A. Wai, "Application of the ManakovPMD equation to studies of signal propagation in optical fibers with randomly varying birefringence," J. Lightwave Technol. 15, 1735-1746 (1997).

[121] C. Menyuk, "Application of multiple-length-scale methods to the study of optical fiber transmission", Journal of Engineering Mathematics, 36, 113-136 (1999).

[122] J. P. Gordon and L. F. Mollenauer, "Phase noise in photonic communications systems using linear amplifiers," Opt. Lett. 15 1351-1353 (1990).

[123] S.K. Turitsyn, T. Schafer, K.H. Spatschek, V.K. Mezentsev, "Path-averaged chirped optical soliton in dispersion-managed fiber communication lines," Optics Communications 163, 122-158 (1999). 
[124] J.D. Ania-Castañón, T. J. Ellingham, R. Ibbotson, X. Chen, L. Zhang, S. K. Turitsyn, "Ultralong Raman fibre lasers as virtually lossless optical media," Phys. Rev. Lett. 96, 023902 (2006);

S. K. Turitsyn, J. D. Ania-Castañón, S. A. Babin, V. Karalekas, P. Harper, D. Churkin, S. Kablukov, A. El-Taher, E. Podivilov, and V. K. Mezentsev, "270-km ultralong Raman fiber laser" Phys. Rev. Lett. 103, 133901 (2009).

[125] M. Ablowitz and H. Segur, Solitons and the Inverse Scattering Transform (SIAM, Philadelphia, 1981).

[126] M. Ablowitz and P. Clarkson, Solitons, Nonlinear Evolution Equations and Inverse Scattering (Cambridge University Press, Cambridge, 1991).

[127] J. M. Geist "Capacity and cutoff rate for dense M-ary PSK constellations," Military Communications Conference, vol. 2, 768 - 770 (1990).

[128] J. P. Aldis and A. G. Burr, "The channel capacity of discrete time phase modulation in AWGN," IEEE Trans. Inf. Theory 39, 184-185 (1993).

[129] K. Ho and J. M. Kahn, "Channel capacity of WDM systems using constantintensity modulation formats," in Optical Fiber Communications Conference, A. Sawchuk, ed., vol. 70 of OSA Trends in Optics and Photonics (Optical Society of America, 2002), paper ThGG85.

[130] A. Lapidoth, "Capacity bounds via duality: A phase noise example," in Proc. 2nd Asian-European Workshop on Information Theory, Breisach, Germany, June 26-29, 2002, pp. 58-61.

[131] A. Lapidoth, "On phase noise channels at high SNR," in Proc. 2002 IEEE Information Theory Workshop, Bangalore, India, Oct. 20-25, 2002.

[132] B. Goebel, R.-J. Essiambre, G. Kramer, P. J. Winzer, and N. Hanik, "Calculation of mutual information for partially coherent Gaussian channels with applications to fiber optics," IEEE Trans. Inf. Theory 57, 5720-5736 (2011).

[133] P. Leoni, S. Calabro, and B. Lank1, "Constellation expansion for 100G transmission," IEEE Photon. Technol. Lett., 25, 1904-1907 (2013). 
[134] E. E. Narimanov and P. Mitra, "The channel capacity of a fiber optics communication system: perturbation theory," Journal of Lightw. Technol. 20, 530-537 (2002).

[135] K. Ho, Phase-modulated optical communication systems. Springer, 2005.

[136] A. P. T. Lau and J. M. Kahn, "Signal design and detection in presence of nonlinear phase noise," J. Lightw. Technol. 25, 3008-3016 (2007).

[137] C. Hager, A. Graell i Amat, A. Alvarado, and E. Agrell, "Design of APSK constellations for coherent optical channels with nonlinear phase noise," IEEE Trans. Commun. 61, 3362-3373 (2013).

[138] A.Mecozzi, "A unified theory of intrachannel nonlinearity in pseudolinear phase-modulated transmission," IEEE Photonics Journal 2, 728 (2010).

[139] J. B. Stark, P. Mitra, and A. Sengupta, "Information capacity of nonlinear wavelength division multiplexing fiber optic transmission line", Optical Fiber Technology 7, 275-288 (2001).

[140] X. Liu, X. Wei, R.E. Slusher, and C.J. McKinstrie, "Improving transmission performance in differential phase-shift-keyed systems by use of lumped nonlinear phase-shift compensation," Opt. Lett. 27, 1616-1618 (2002).

[141] K. Kikuchi, "Electronic post-compensation for nonlinear phase fluctuations in a 1000-km 20-gbit/s optical quadrature phase-shift keying transmission system using the digital coherent receiver," Opt. Express 16(2), 889-896 (2008).

[142] L. F. Mollenauer, A. Grant, X. Liu, X. Wei, C. Xie, I. Kang, and C. Doerr, "Demonstration of 109 X $10 \mathrm{G}$ dense WDM over more than 18,000 km using novel, periodic-group-delay complemented dispersion compensation and dispersion managed solitons," in Proceedings of ECOC 03, Rimini, Post-deadline Paper Th4.3.4, 2003.

[143] D. Rafique and A. D. Ellis, "Impact of signal-ASE four-wave mixing on the effectiveness of digital back-propagation in $112 \mathrm{~Gb} / \mathrm{s}$ PM-QPSK systems", Optics Express, 19, 3449-3453 (2011). 
[144] T. Tanimura, M. Nolle, J.K. Fischer, and C. Schubert, "Analytical results on back propagation nonlinear compensator with coherent detection," Opt. Express 20, 28779-28785 (2012).

[145] F. Parmigiani, L. Provost, P. Petropoulos, D.J. Richardson, W. Freude, J. Leuthold, A. D. Ellis, and I. Tomkos, "Progress in multichannel all-optical regeneration based on fiber technology", IEEE J. Select. Topics in Quantum Electron. 18, 689-700 (2012).

[146] M. Vasilyev, and T. I. Lakoba, "All-optical multichannel 2R regeneration in a fiber-based device", Opt. Lett. 30, 1458-1460 (2005).

[147] T. I. Lakoba, and M. Vasilyev, "A new robust regime for a dispersion-managed multichannel regenerator", Opt. Exp. 15, 10061-10074 (2007).

[148] J. Kakande, R. Slavik, F. Parmigiani, A. Bogris, D. Syvridis, L. Gruner-Nielsen, R. Phelan, P. Petropoulos and D. J. Richardson, "Multilevel quantization of optical phase in a novel coherent parametric mixer architecture," Nat. Photonics 4(12), 748-752 (2011).

[149] T. Widdowson, J.P. Hueting, A.D. Ellis, D.J. Malyon and P.J. Watkinson, "Global fibre transmission using optically amplified regenerators for maximised repeater spacing, "Electronics Letters 30, 2056-2057 (1994).

[150] O. Leclerc,U E. Desurvire, and O. Audouin, "Synchronous WDM soliton regeneration: toward 80-160 Gbit/s transoceanic systems," Optical Fiber Technology 3(2), 97-116 (1997).

[151] A. D. Ellis and J. Zhao, "Channel capacity of non-linear transmission systems," in Impact of Nonlinearities on Fiber Optic Communications, S. Kumar, Ed. New York, NY: Springer, 2011, ch. 13, pp. 507-538.

[152] K. Croussore, C. Kim, and G. Li, "All-optical regeneration of differential phase shift keying signals based on phase-sensitive amplification," Opt. Lett. 29(20), 2357-2359 (2004). 
[153] J.M. Martinis, Devoret M.H., and Clarke J. "Energy-level quantization in the zero-voltage state of a current-biased Josephson junction", Phys. Rev. Lett. 55, 1543-1546 (1985).

[154] K. Lindenberg, J. M. Sancho, A. M. Lacasta, and I. M Sokolov, "Dispersionless transport in a washboard potential", Phys. Rev. Lett. 98, 020602 (2007).

[155] D. Angeli, J.F. Ferrell, and E.D. Sontag, "Detection of multistability, bifurcations, and hysteresis in a large class of biological positive-feedback systems", Proc. Nat. Acad. Sci. USA 101, 1822-1827 (2004).

[156] J. R. Crutchfield, M. Nauenberg, and J. Rudnick, "Scaling for external noise at the onset of chaos," Phys. Rev. Lett. 46, 933-935 (1981).

[157] P. Reimann and P. Talkner, "Probability densities for discrete dynamical systems with weak noise," Hel. Phys. Acta 63, 845-846 (1991).

[158] http://www.mathworks.co.uk/help/optim/ug/constrained-nonlinearoptimization-algorithms.html (Matlab2014a documentation)

[159] R. Tkach, "Scaling optical communications for the next decade and beyond," Bell Labs Techn. J. 14(4), 3-9 (2010).

[160] T. Roethlingshoefer, G. Onishchukov, B. Schmauss, and G. Leuchs, "Multilevel amplitude and phase regeneration in a nonlinear amplifying loop mirror with a phase-Sensitive Amplifier," in Proceedings of European Conference on Optical Communication (ECOC 2012), Amsterdam, paper Tu.1.A.3 (2012).

[161] S. Sygletos, P. Frascella, S.K. Ibrahim, L. Gruner-Nielsen, R. Phelan, J. O'Gorman, and A.D. Ellis, "A practical phase sensitive amplification scheme for two channel phase regeneration," Opt. Express 19(26), 938-945 (2011).

[162] L. Beygi, E. Agrell, and M. Karlsson, "On the optimization of 16-point ring constellations in the presence of nonlinear phase noise," in Proceedings of Optical Communication Conference, 2011 OSA Technical Digest Series (Optical Society of America), paper OThO4 (2011). 
[163] H. J. Thiele, A. D. Ellis, and I. D. Phillips, "Recirculating loop demonstration of $40 \mathrm{Gbit} / \mathrm{s}$ all-optical 3R data regeneration using a semiconductor nonlinear interferometer," Elect. Lett. 35(3), 230-231 (1990).

[164] I. D. Phillips, A. Gloag, P. N. Kean, N. J. Doran, I. Bennion, and A. D. Ellis, "Simultaneous de-multiplexing, data regeneration and clock recovery using a single semiconductor optical amplifier based nonlinear optical loop mirror," Opt. Lett. 22(17), 1326-1328 (1997).

[165] I. Kang, C. Dorrer, L. Zhang, M. Rasras, L. Buhl, A. Bhardwaj, S. Cabot, M. Dinu, X. Liu, M. Cappuzzo, L. Gomez, A. Wong-Foy, Y. F. Chen, S. Patel, D. T. Neilson, J. Jacques, and C. R. Giles, "Regenerative all optical wavelength conversion of $40 \mathrm{Gbit} / \mathrm{s}$ DPSK signals using a SOA MZI," in Proceedings of European Conference on Optical Communication (ECOC 2005), Amsterdam, paper Thu 4.3.3 (2005).

[166] D. Wolfson, P. B. Hansen, A. Kloch, T. Fjelde, C. Janz, A. Coquelin, I. Guillemont, F. Gaborit, and M. Renaud, "All-optical 2R regeneration at $40 \mathrm{Gbit} / \mathrm{s}$ in an SOA-based Mach-Zehnder interferometer," in Proceedings of Optical Communication Conference, Beijing, 456-457 (1999).

[167] S. Boscolo, K. J. Blow, and S. K. Turitsyn, "All-optical passive 2R regeneration for Nx40 Gbit/s WDM transmission using NOLM and novel filtering technique," Opt. Commun. 217, 227-232 (2003).

[168] M. A. Sorokina and S. K. Turitsyn, "Regeneration limit of classical Shannon capacity," Nat. Comm. 5 (2014).

[169] J. Kakande, R. Slavik, F. Parmigiani, P. Petropoulos, and D. Richardson, "Alloptical processing of multi-level phase shift keyed signals", in Optical Fiber Communication Conference, OSA Technical Digest (Optical Society of America, 2012), paper OW1I.3.

[170] R.M. May, "Simple mathematical models with very complicated dynamics", Nature 261, 459-467 (1976). 
[171] M. A. Sorokina, S. Sygletos, and S. K. Turitsyn, "Optimization of cascaded regenerative links based on phase sensitive amplifiers," Opt. Lett. 38 (20), 43784381 (2013).

[172] E. Desurvire, D. Bayart, B. Desthieux, and S. Bigo, Erbium-doped fiber amplifiers: Device and System Developments, (J. Wiley and Sons, 2002).

[173] X. Chen and W. Shieh, "Closed-form expressions for nonlinear transmission performance of densely spaced coherent optical OFDM systems," Opt. Express 18(18), 19039-19054 (2012).

[174] D. Rafique and A. D. Ellis, "Impact of signal-ASE four wave mixing on the effectiveness of digital back-propagation in $112 \mathrm{Gbit} / \mathrm{s}$ PM-QPSK systems," Opt. Express 19(4), 3449-3454 (2011).

[175] E. Torrengo, R. Cigliutti, G. Bosco, A. Carena, V. Curri, P. Poggiolini, A. Nespola, D. Zeolla, and F. Forghieri, "Experimental validation of an analytical model for nonlinear propagation in uncompensated optical links," in Proceedings of European Conference on Optical Communication (ECOC 2011), Amsterdam, paper We.7.B.2 (2011).

[176] M. C. Jeruchim, "Techniques of estimating the bit error rate in the simulation of digital communication systems," IEEE J. Sel. Top. Quantum Electron. 2(1), 153-170 (1984).

[177] N. J. Doran and David Wood, "Nonlinear-optical loop mirror," Opt. Lett. 13, 56-58 (1988).

[178] S. Boscolo, S. K. Turitsyn, and K. J. Blow, "Nonlinear loop mirror-based alloptical signal processing in fiber-optic communications," Optical Fiber Technology 14, 299-316 (2008).

[179] G.J. Foschini, N.J. Holmdel, R. Gitlin, and S. Weinstein, "Optimization of Two-Dimensional Signal Constellations in the Presence of Gaussian Noise," IEEE,Transactions on Communications 22(1), 28-38 (1974). 
[180] Z. H. Peric, I. B. Djordjevic, S. M. Bogosavljevic, and M. C. Stefanovic, "Design of signal constellations for Gaussian channel by iterative polar quantization," in Proc. 9th Mediterranean Electrotech. Conf. 2, 866-869 (1998).

[181] R. Dischler, "Experimental Study of 16-, 32- and 64-QAM Constellation Sets in the 200-Gb/s Regime on a Data Rate Flexible System," in Optical Fiber Communication Conference/National Fiber Optic Engineers Conference 2013, OSA Technical Digest (online) (Optical Society of America, 2013), paper OTh3B.2.

[182] M. Sorokina, S. Sygletos, A. D. Ellis, and S. Turitsyn, "Optimal packing for cascaded regenerative transmission based on phase sensitive amplifiers," Opt. Express 21, 31201-31211 (2013). 